GROUND-WATER RESOURCES OF THE CAGUAS-JUNCOS VALLEY, PUERTO RICO

by Juan C. Puig and José M. Rodríguez

with a section entitled, "General Geology" by Jesús Rodríguez-Martínez

U.S. GEOLOGICAL SURVEY

Water-Resources Investigations Report 91-4079

Prepared in cooperation with the

PUERTO RICO DEPARTMENT OF NATURAL RESOURCES

PUERTO RICO AQUEDUCT AND SEWER AUTHORITY

San Juan, Puerto Rico

1993 


\section{U.S. DEPARTMENT OF THE INTERIOR MANUEL LUJAN, Jr., Secretary \\ U.S. GEOLOGICAL SURVEY \\ Dallas I. Peck, Director}

For additional information write to:

District Chief

U.S. Geological Survey

P.O. Box 364424

San Juan, Puerto Rico 00936-4424
Copies of this report can be purchased from:

U.S. Geological Survey

Books and Open-File Reports Section

Federal Center

Box 25425, Denver, CO 80225 


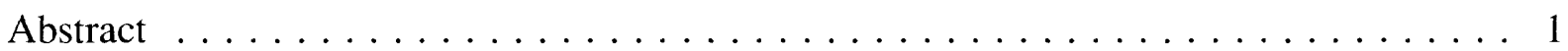

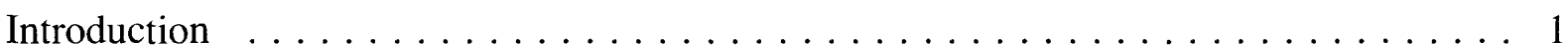

Purpose and scope $\ldots \ldots \ldots \ldots \ldots \ldots \ldots \ldots \ldots \ldots \ldots \ldots \ldots \ldots \ldots \ldots$

Physical setting $\ldots \ldots \ldots \ldots \ldots \ldots \ldots \ldots \ldots \ldots \ldots \ldots \ldots \ldots$

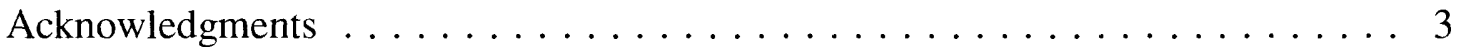

General geology $\ldots \ldots \ldots \ldots \ldots \ldots \ldots \ldots \ldots \ldots \ldots \ldots \ldots \ldots \ldots \ldots \ldots \ldots \ldots$

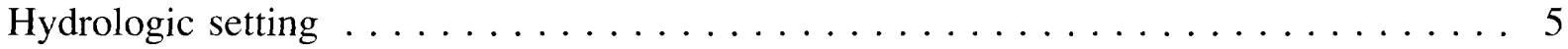

Rainfall and evapotranspiration $\ldots \ldots \ldots \ldots \ldots \ldots \ldots \ldots$

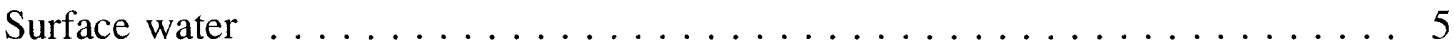

Ground-water hydrology $\ldots \ldots \ldots \ldots \ldots \ldots \ldots \ldots \ldots \ldots \ldots \ldots \ldots \ldots \ldots \ldots \ldots$

Water wells and use $\ldots \ldots \ldots \ldots \ldots \ldots \ldots \ldots \ldots \ldots \ldots \ldots \ldots \ldots$

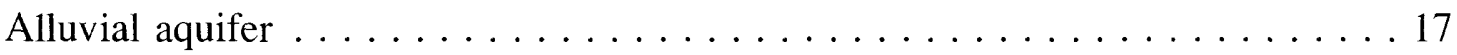

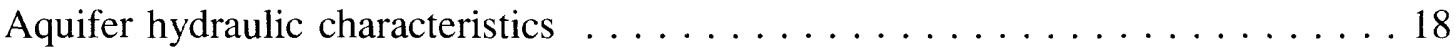

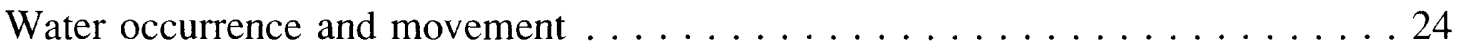

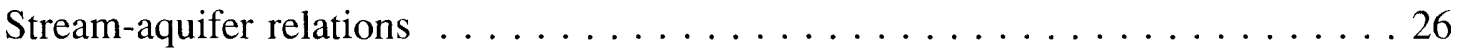

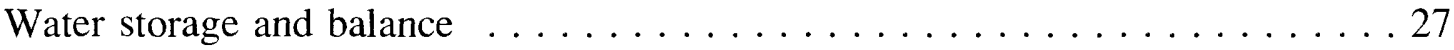

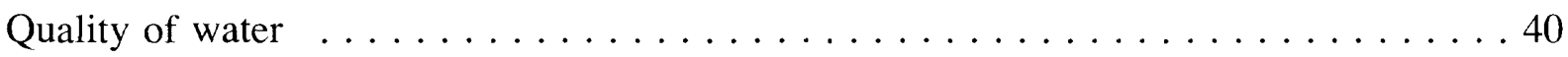

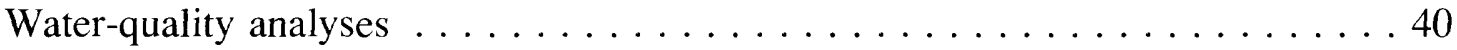

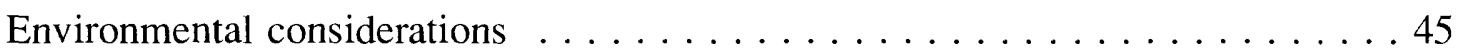

Ground-water development and management considerations $\ldots \ldots \ldots \ldots \ldots$. . . 47

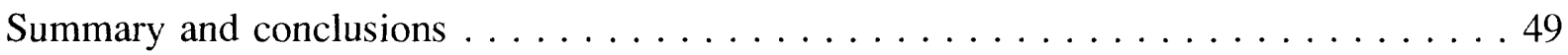

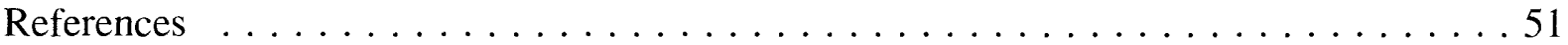




\section{ILLUSTRATIONS}

Page

\section{Figure}

1-3. Map showing:

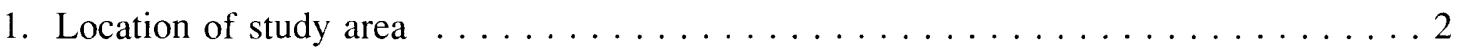

2. Generalized surficial geology of the study area $\ldots \ldots \ldots \ldots \ldots \ldots \ldots$

3. Precipitation for the upper Río Grande de Loíza basin during the 1986 water year . . . . . . . . . . . . . . . . . . . . . . 6

4-6. Graph showing:

4. Monthly precipitation at Caguas $1 \mathrm{~W}$, Gurabo Substation, and Juncos 1NNE, $1986-1988 \ldots \ldots \ldots \ldots \ldots \ldots \ldots \ldots \ldots$

5. Monthly pan evaporation at Gurabo Agricultural Experimental

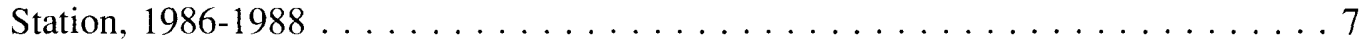

6. Streamflow hydrographs for (a) Río Valenciano at Juncos and Río Gurabo at Gurabo, 1985-1988, and (b) Río Turabo at Borinquen, and Río Grande de Loíza at Caguas, 1985-1988

7. Map showing the location of data collection sites, landfills, and water

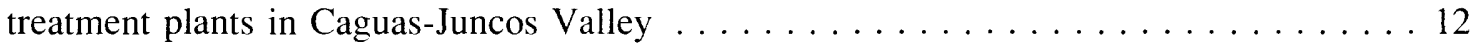

8. Graph showing flow-duration curves for Río Grande de Loíza at Caguas,

Río Gurabo at Gurabo, and Río Valenciano at Juncos $\ldots \ldots \ldots \ldots \ldots$

9. Map showing thickness of alluvial deposits and location of hydrogeologic sections $\mathrm{A}-\mathrm{A}^{\prime}$ and $\mathrm{B}-\mathrm{B}^{\prime}$

10. Hydrogeologic sections (a) A-A' along Caguas subarea, and (b) B-B'

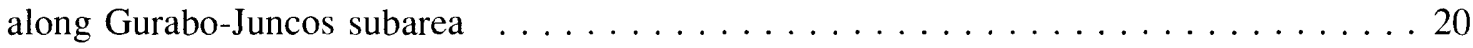

11-13. Map showing:

11. Transmissivity distribution in the alluvial aquifer $\ldots \ldots \ldots \ldots \ldots \ldots \ldots$

12. Potentiometric surface of the alluvial aquifer for July $27-29,1987 \ldots \ldots \ldots$

13. Potentiometric surface and stream discharges within the alluvial

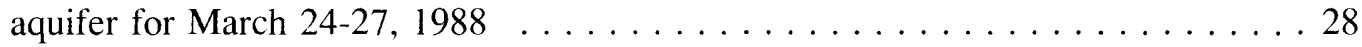

14. Graph showing relation between land surface and the potentiometric surface along regional flow paths in the Caguas and Gurabo-Juncos subareas, March 24-27, 1988

15. Hydrographs of water levels in wells 90 CJ-TW 09 and 36 Manrique 1, $1987-1988$

16. Hydrographs of water levels in wells 84 CJ-TW 3B and 17 Gurabo-USGS,

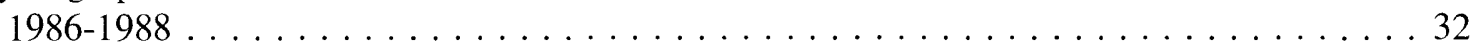

17. Hydrographs of water levels in shallow and deep wells along hydrogeologic section B-B' near Juncos showing the direction of vertical movement of water . . . . . 33 


\section{ILLUSTRATIONS}

\section{Figure}

18-21. Hydrographs of periodic ground-water levels for:

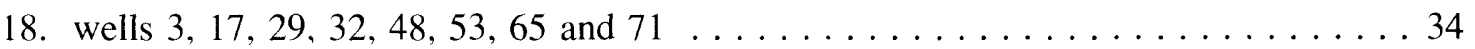

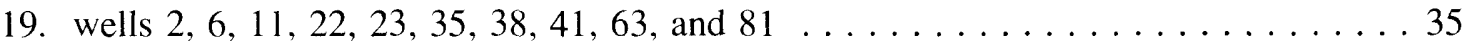

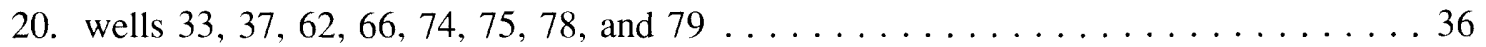

21. wells $49,82,83,84,85,86,87,88,90$, and $91 \ldots \ldots \ldots \ldots \ldots \ldots \ldots \ldots \ldots$

22. Diagram showing major ion composition of ground water and surface

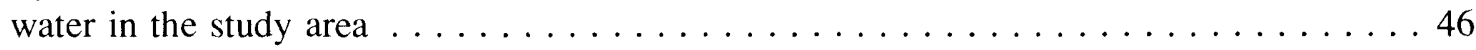

\section{TABLES}

Table

1. Description of wells inventoried in the Caguas-Juncos Valley $\ldots \ldots \ldots \ldots \ldots \ldots$

2. Ground-water withdrawals from the alluvial aquifer in the Caguas-Juncos

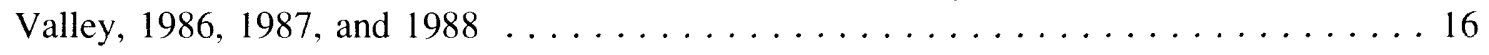

3. Transmissivity estimates from specific-capacity data in the Caguas-Juncos Valley . . . . 22

4. Transmissivity estimates from slug-test data in the Caguas-Juncos Valley . . . . . . . . 22

5. Estimated average interstitial velocities at selected locations throughout

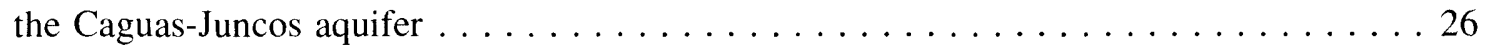

6. Results of the seepage study conducted along the principal streams flowing through the Caguas-Juncos Valley, March 25, $1988 \ldots \ldots \ldots \ldots$

7. Estimated steady-state water budgets of the Caguas and the Gurabo-Juncos

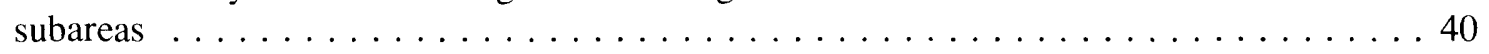

8. Physical and chemical characteristics of ground water at selected wells

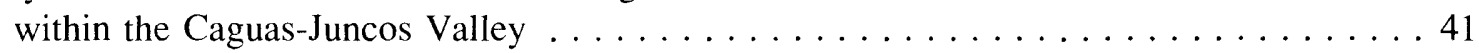

9. Biological characteristics of ground water at selected wells within the Caguas-

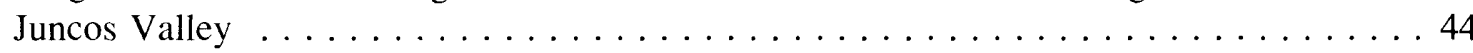

10. Physical, chemical, and biological characteristics of water at selected streams within the Caguas-Juncos Valley 


\section{Multiply}

inch (in.)

foot (ft)

mile (mi)

square mile $\left(\mathrm{mi}^{2}\right)$

acre-foot (acre-ft)

cubic foot per second $\left(\mathrm{ft}^{3} / \mathrm{s}\right)$

gallon per minute ( $\mathrm{gal} / \mathrm{min}$ )

million gallons per day $(\mathrm{Mgal} / \mathrm{d})$

gallon per minute per foot (gal/min)/ft

foot per day (ft/d)

foot squared per day $\left(\mathrm{ft}^{2} / \mathrm{d}\right)$
By

To obtain

\section{Length}

25.4

0.3048

1.609

$\underline{\text { Area }}$

2.590

\section{Volume}

1233

\section{Flow}

0.02832

0.06308

3.785

\section{Specific capacity}

0.2070

liter per second per meter

\section{Hydraulic conductivity}

0.3048

meter per day

\section{Transmissivity}

0.09290 millimeter

meter

kilometer

square kilometer

cubic meter

cubic meter per second

liter per second

million liters per day

Temperature: Temperature in degrees Celsius $\left({ }^{\circ} \mathrm{C}\right)$ may be converted to degrees Fahrenheit $\left({ }^{\circ} \mathrm{F}\right)$ as follows:

$$
{ }^{\circ} \mathrm{F}=1.8 \times{ }^{\circ} \mathrm{C}+32
$$

\section{Abbreviated water-quality units used in report:}

colonies per 100 milliliters (col./100 ml)

micrograms per liter $(\mu \mathrm{g} / \mathrm{L})$

milligrams per liter $(\mathrm{mg} / \mathrm{L})$

microsiemens per centimeter at $25^{\circ} \mathrm{C}(\mu \mathrm{S} / \mathrm{cm})$

\section{Acronyms used in report:}

Puerto Rico Aqueduct and Sewer Authority (PRASA)

Puerto Rico Department of Natural Resources (PRDNR)

U.S. Environmental Protection Agency (EPA)

U.S. Geological Survey (USGS) 


\title{
GROUND-WATER RESOURCES OF THE CAGUAS-JUNCOS VALLEY, PUERTO RICO
}

\author{
By Juan C. Puig and José M. Rodríguez
}

\section{ABSTRACT}

The Caguas-Juncos Valley, which occupies an area of about 35 square miles in east-central Puerto Rico, is underlain by a largely unconfined alluvial aquifer that consists of clay, silt, fine-grained sand, and subordinate amounts of coarse-grained sand and gravel. The alluvial aquifer has a maximum thickness of about 200 feet. Ground water in the alluvial aquifer flows from the recharge areas toward the principal streams. The direction of regional flow is primarily from east to west in the Gurabo-Juncos subarea and southwest to northeast in the Caguas subarea toward Lago Loiza along the main flood plains. Ground-water withdrawals from the alluvial aquifer totaled about 3.0 million gallons per day in 1988. Some wells completed in the alluvial aquifer near Gurabo and Juncos yield as much as 310 gallons per minute. The average well yield in Gurabo and Juncos is about 85 gallons per minute, and the average yield in Caguas is about 28 gallons per minute. The potentiometric surface of the alluvial aquifer varies seasonally, with the highest levels generally occurring near the end of December and the lowest levels occurring in April.

Transmissivity of the alluvial aquifer ranges from 66 to 4,770 feet squared per day near stream channels. The estimated specific yield of the water-table aquifer is about 10 to 15 percent. The amount of water stored in the aquifer was estimated to be about 122,000 acre-feet.

Ground-water quality analyses revealed the presence of two distinct problems: high natural concentrations of iron and manganese, and localized areas of human-related contamination. The problems were not widespread and many areas had ground water of suitable quality for most uses. Analysis of both ground water and surface water shows that, in general, the waters are of the calcium-bicarbonate type and typically have dissolved solids concentrations of less than 500 milligrams per liter.

\section{INTRODUCTION}

The Caguas-Juncos Valley, located in the east-central part of Puerto Rico (fig. 1), is the largest interior valley on the island and covers approximately $35 \mathrm{mi}^{2}$. The valley is part of the upper Río Grande de Loíza basin, which covers an area of about $210 \mathrm{mi}^{2}$.

The Caguas-Juncos Valley has a natural abundance of water, but a great deal of the water is exported to San Juan. Because of the rapid population growth in the valley, there is concern that additional water supplies may be needed to meet future demand. Even though relatively little is known about the ground-water resources of the valley, they are adequate to supplement the existing surface-water supplies. In order to assess the potential for ground-water development in the valley, the U.S. Geological Survey (USGS), in cooperation with the Puerto Rico Department of Natural Resources (PRDNR) and the Puerto Rico Aqueduct and Sewer Authority (PRASA), conducted an investigation of the ground-water resources of the Caguas-Juncos Valley from 1986 through 1989.

\section{Purpose and Scope}

The purpose of this report is to document the occurrence, availability, and quality of the ground water in the Caguas-Juncos Valley. The report also discusses management considerations for ground-water development and the protection of water quality. Because of the lack of historical hydrologic data, this investigation is based mainly on hydrologic data collected between 1986 and 1988. The hydrologic data collected included ground-water levels and withdrawal rates, aquifer hydraulic characteristics and physical boundaries, precipitation, streamflow, and surface-water use. Water-quality data also were collected from public-supply wells. These data included physical characteristics and concentrations of major anions and cations, nutrients, and organic compounds. This report evaluates the potential for ground-water development within the Caguas-Juncos Valley. The study represents the first effort to conceptualize and quantify the ground-water resources of the area. The hydrologic information presented here will serve as a basis upon which a ground-water flow model could be calibrated in the future.

\section{Physical Setting}

The Caguas-Juncos Valley is divided into two distinct subareas: (1) the approximately circular Caguas subarea to the west, which covers an area of about $16.6 \mathrm{mi}^{2}$, and (2) the elongate Gurabo-Juncos 


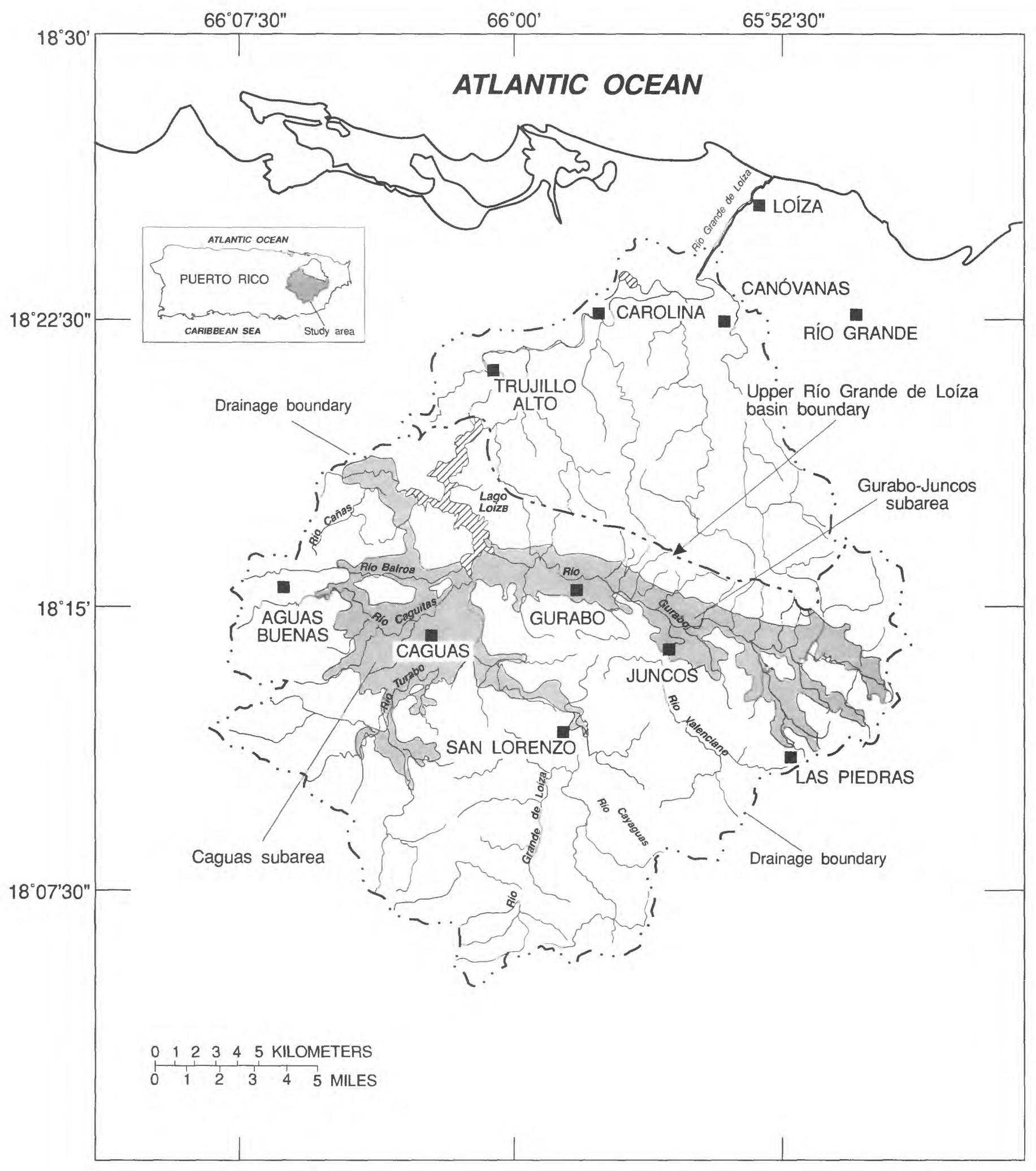

Figure 1.--Location of study area. 
subarea to the east, which covers an area of about 18.5 $\mathrm{mi}^{2}$ (fig. 1). The subareas are connected along a 3,500 ft reach of Río Grande de Loíza just upstream of Lago Loíza. The Caguas subarea has an average diameter of about $4 \mathrm{mi}$. The Caguas subarea floor is steep and irregular with a maximum altitude of about $490 \mathrm{ft}$ above mean sea level south of Caguas, and a minimum altitude of about $145 \mathrm{ft}$ near Lago Loíza. In contrast, the Gurabo-Juncos subarea floor is a flat. narrow plain about $12 \mathrm{mi}$ long and from 0.5 to $1.5 \mathrm{mi}$ wide. The Gurabo-Juncos subarea has a maximum altitude of about $360 \mathrm{ft}$ at the eastern boundary and a minimum altitude of about $145 \mathrm{ft}$ near Lago Loíza.

The principal streams traversing the Caguas subarea are the: Río Grande de Loíza from south to north along the eastern edge; Río Turabo from west to east in the southern part of the valley; Río Caguiitas from west to east in the central part of the valley; and Río Bairoa from west to east along the northern part of the valley. Two main streams flow through the Gurabo-Juncos subarea: (1) the Río Gurabo flows from east to west along the center of the valley, and (2) the lower reach of the Río Valenciano, which flows from south to north near Juncos.

The Caguas-Juncos Valley, as described in this report, includes the cities of Caguas, Gurabo, and Juncos, which had a combined population of about 167,000 in 1980 and an estimated population of 192,000 in 1990 (Puerto Rico Planning Board, 1984). The main urban area, Caguas, is built on alluvial deposits, while the cities of Gurabo and Juncos are located at the southern limits of the alluvial plain, outside the flood plain. The Caguas subarea is about 40 percent urbanized, while the Gurabo-Juncos subarea is mostly pasture land (90 percent).

\section{Acknowledgments}

The authors are grateful to personnel of the Puerto Rico Aqueduct and Sewer Authority at Humacao, Caguas, and Guaynabo who assisted with the water-quality sampling of the public water-supply wells, and who provided valuable information about water use and access to the well construction files. The authors also acknowledge the assistance of land owners in Caguas, Gurabo, and Juncos who permitted the installation of piezometers on their property.

\section{GENERAL GEOLOGY}

by Jesús Rodríguez-Martínez

The geology of the Caguas-Juncos Valley has been described by Pease (1968), Rogers (1979), Seiders (1971), Broedel (1961), and McGonigle (1978). The rocks constituting the basement and flanks of this valley are composed largely of volcaniclastics, lavas, intrusives, minor amounts of metamorphic rock of Late Cretaceous to early Tertiary age, and locally minor amounts of limestone of early Tertiary age. These rocks are overlain by surface deposits, mainly of alluvial origin during the Holocene (fig. 2).

The volcanic rocks (volcaniclastics and lavas) are the most abundant rocks in the study area. The volcaniclastic rocks (tuffaceous sandstone, siltstone, volcanic conglomerate, and breccia) formed from the deposition of primary or reworked volcanogenic material in marine waters. The intercalation of the volcaniclastic rocks with lavas represents changes in the type of volcanism and irregular periods of volcanic quiescence.

The volcaniclastic rock group is complex and has been differentiated into numerous units by some investigators. The stratigraphic relations among the different units of this group and with the other rock types have not been completely established, due to the intensity of faulting and variations in the different facies. These rocks will be described in this section as a group and no attempt will be made to differentiate among them.

Irregularly distributed intrusive rocks in the form of stocks, dikes, and sills of varying mineralogical composition have intruded the volcanic rocks. Part of the San Lorenzo Batholith crops out near Caguas. A minor body of limestone, the Aguas Buenas Limestone Member of the Torrecilla Breccia (Early Cretaceous age), is found in the study area. The areal extent of the Aguas Buenas Limestone Member is too small to be included in figure 2. Metamorphic rocks are associated with the emplacement of the intrusive bodies. These metamorphic rocks are quantitatively insignificant, although some enriched mineral zones of unknown importance are encountered adjacent to them.

The Late Cretaceous and early Tertiary rocks are highly faulted, and locally folded. The faults are mostly normal, although a significant strike-slip component has been observed in some of them. The 


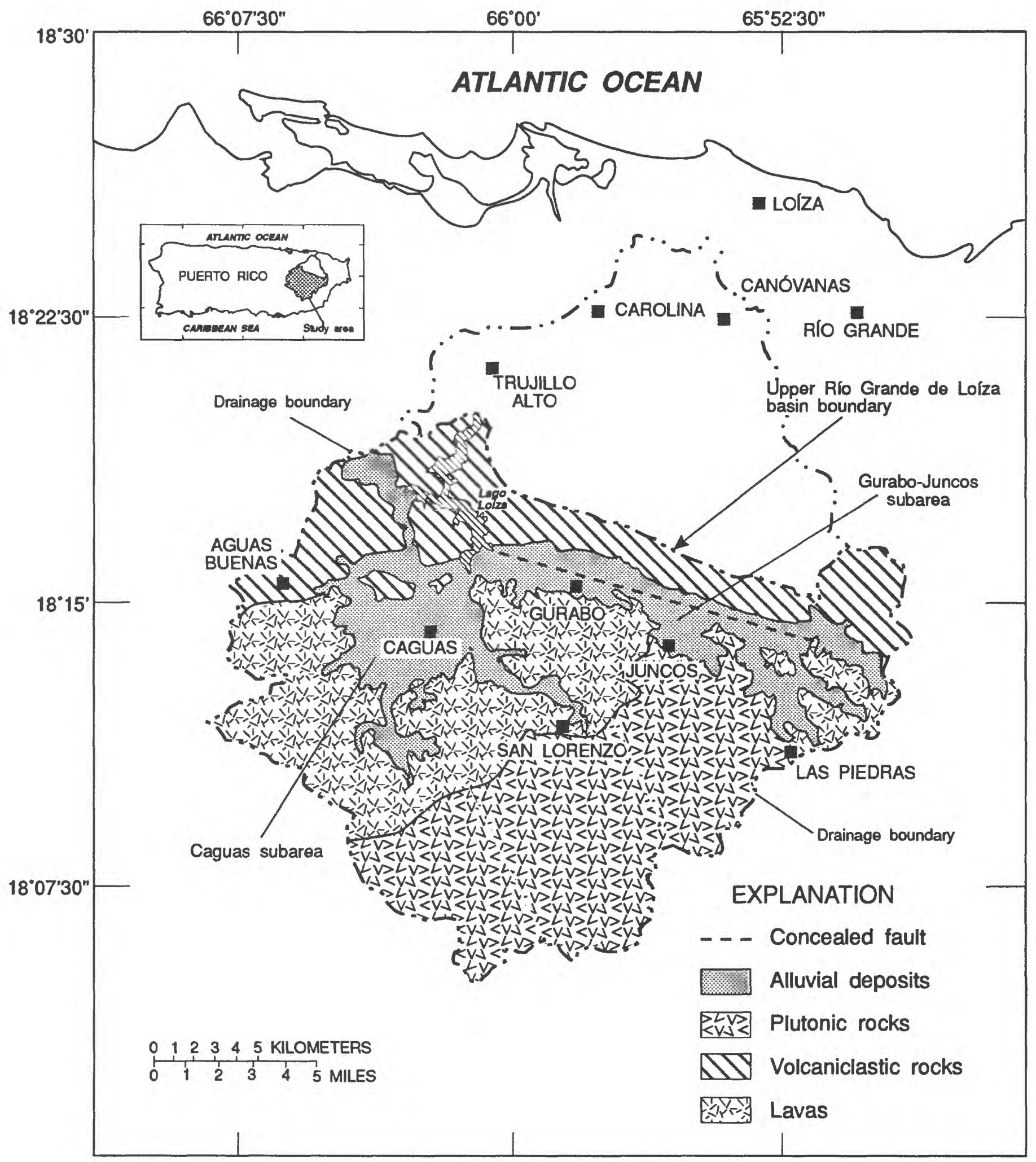

Figure 2.--Generalized surficial geology of the study area. 
strike of the faults is predominantly southeast to north-northwest. Locally, the development of the faults is presumed to have been closely related to the emplacement of intrusive rocks. Extensive systems of fractures and joints, regularly filled with calcite, quartz, and sericite that were emplaced by both faulting and precipitation from hydrothermal solutions associated with the late stages of volcanism, are present.

The surface material is of a predominantly alluvial origin and consists of varying lithologies, which reflect the changing nature of the source material and the dynamics of the fluvial history of the rivers that drain the enclosing basin. In the area of Caguas, the alluvium is mostly composed of silt, clay, and fine-grained sand, with subordinate amounts of gravel and coarse-grained sand. The amount of gravel and coarse sand in the alluvium is greater in the Gurabo-Juncos area than in the Caguas area.

The volcaniclastic and other consolidated rock groups are of little hydrologic importance, because they have insufficient permeability to be major waterbearing units. Where they are fractured, however, perennial and ephemeral low-flow springs have been encountered.

\section{HYDROLOGIC SETTING}

Ground-water flow constitutes an important component in the hydrologic cycle. The importance of the ground-water flow component is best appraised if it is compared with other hydrologic cycle components, such as rainfall, evapotranspiration, and runoff. A brief overview of these hydrologic components follows in order to provide data for such a comparison.

In the Caguas-Juncos Valley, hydrologic parameters, such as rainfall and surface runoff, can change dramatically within a short period of time; however, temperatures fluctuate little. Between 1986 and 1988 , monthly average temperature varied only about $8{ }^{\circ} \mathrm{F}$ from a minimum of $72.9^{\circ} \mathrm{F}$ (January to February) to a maximum of $80.6^{\circ} \mathrm{F}$ (August).

\section{Rainfall and Evapotranspiration}

Precipitation in the upper Río Grande de Loíza basin, during the 1986 water year (October 1985 through September 1986), ranged from about 65 to 80 inches in the valley and from 80 to 110 inches in the highlands (fig. 3). Although there is no definite pattern of rainfall distribution in time, November is typically a wet month and April is typically a dry month. Precipitation during 1987 was above normal, totalling about 74 inches in Caguas, 85 inches in Gurabo, and 98 inches in Juncos (fig. 4). However, annual precipitation totals from 1986 through 1988 averaged about 62 inches in Caguas, 70 inches in Gurabo, and 76 inches in Juncos (fig. 4). The lowest monthly precipitation recorded from 1986 through 1988 was 0.22 inches during July 1987 at Caguas. The highest monthly value recorded for the same period was 25.16 inches at Juncos during November of 1987.

Water, as soil moisture, is absorbed by vegetation and transpired from plant surfaces, and escapes to the atmosphere through direct evaporation from exposed soil surfaces. The combined effect of both processes is known as evapotranspiration.

The evapotranspiration (ET) rate is difficult to quantify; however, it can be estimated. Potential ET, that is, evapotranspiration from an extensive, closed, homogeneous cover of vegetation that has a sufficient amount of water, is usually assumed to be about 70 percent of the recorded pan evaporation (Bennet, 1976). The average annual pan evaporation recorded at the Gurabo experimental station from 1986 through 1988 was 68 inches (fig. 5); therefore, potential ET may be about 48 in/yr near Gurabo.

The evapotranspiration was also computed as the residual of weighted mean-annual rainfall volume minus the runoff volume from basins within the volcanic interior of Puerto Rico (Giusti and López, 1967). This analysis was based on surface water gaging stations throughout Puerto Rico in basins where the change in aquifer storage can be considered negligible. Giusti and López used this relationship to estimate the average annual runoff from basins within the volcanic interior to be about 45 percent of the mean-annual rainfall, and the average evapotranspiration is the remaining 55 percent.

Using this method, ET is estimated to be from about 34 to $42 \mathrm{in} / \mathrm{yr}$ in the Caguas-Juncos Valley based on 1986 through 1988 precipitation levels of 62 to 76 in/yr. To provide a more precise ET value would require the application of equations based on an energy balance, and such an approach is beyond the scope of this study.

\section{Surface Water}

Surface water in the Caguas-Juncos Valley is abundant, and numerous streams and creeks traverse 


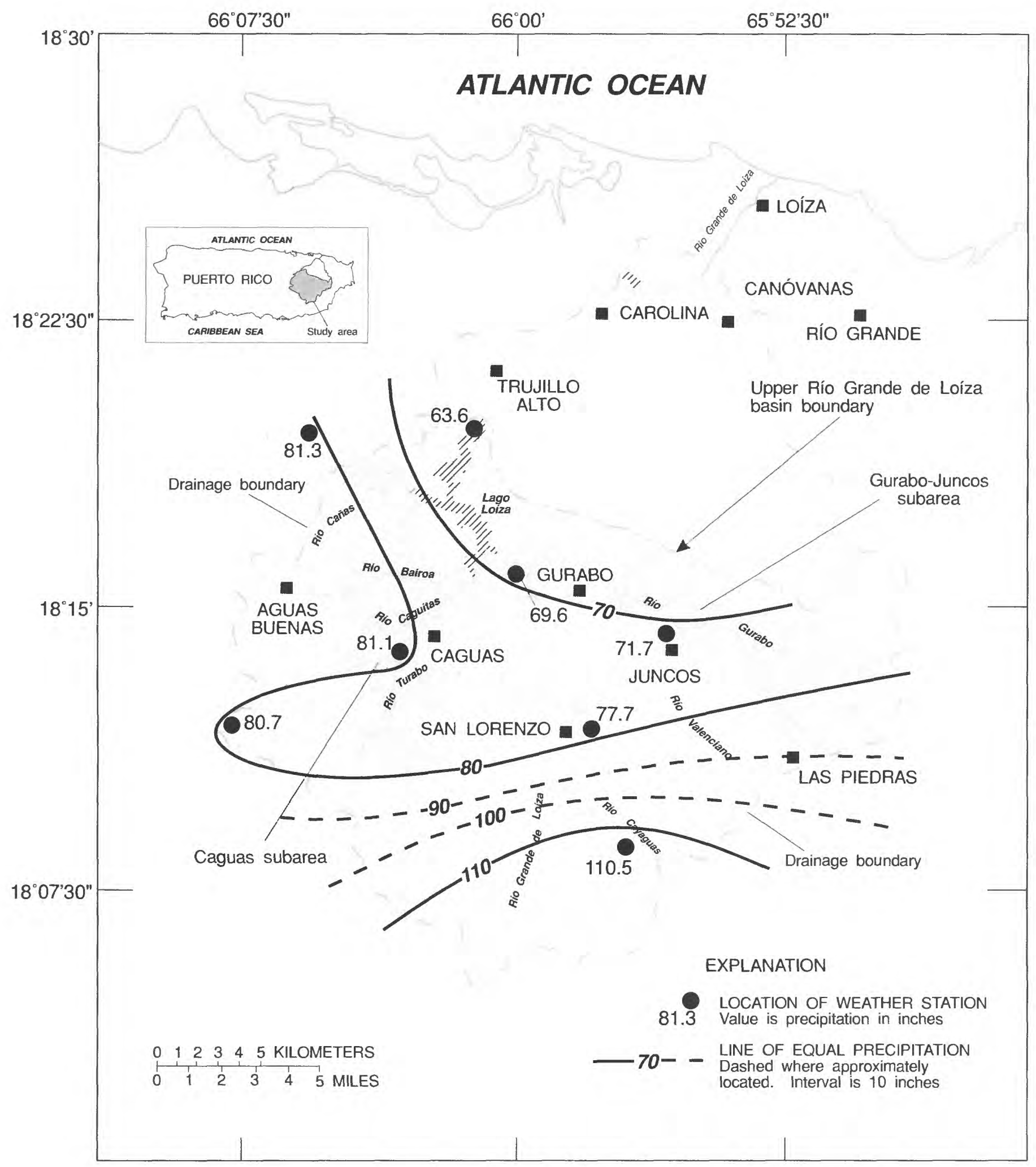

Figure 3.--Precipitation for the upper Río Grande de Loíza basin during the 1986 water year. 


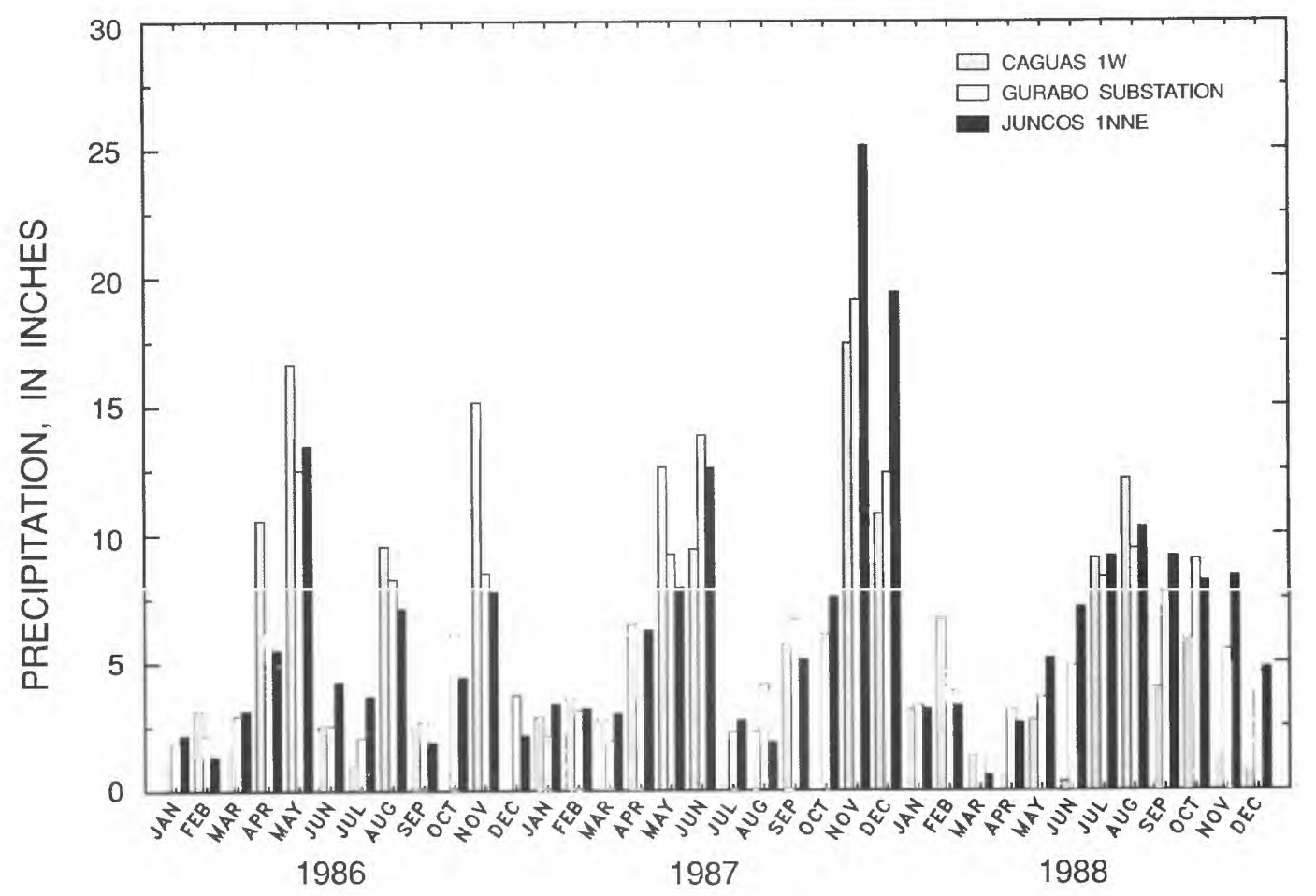

Figure 4.--Monthly precipitation at Caguas 1W, Gurabo Substation, and Juncos 1NNE, 1986-1988.

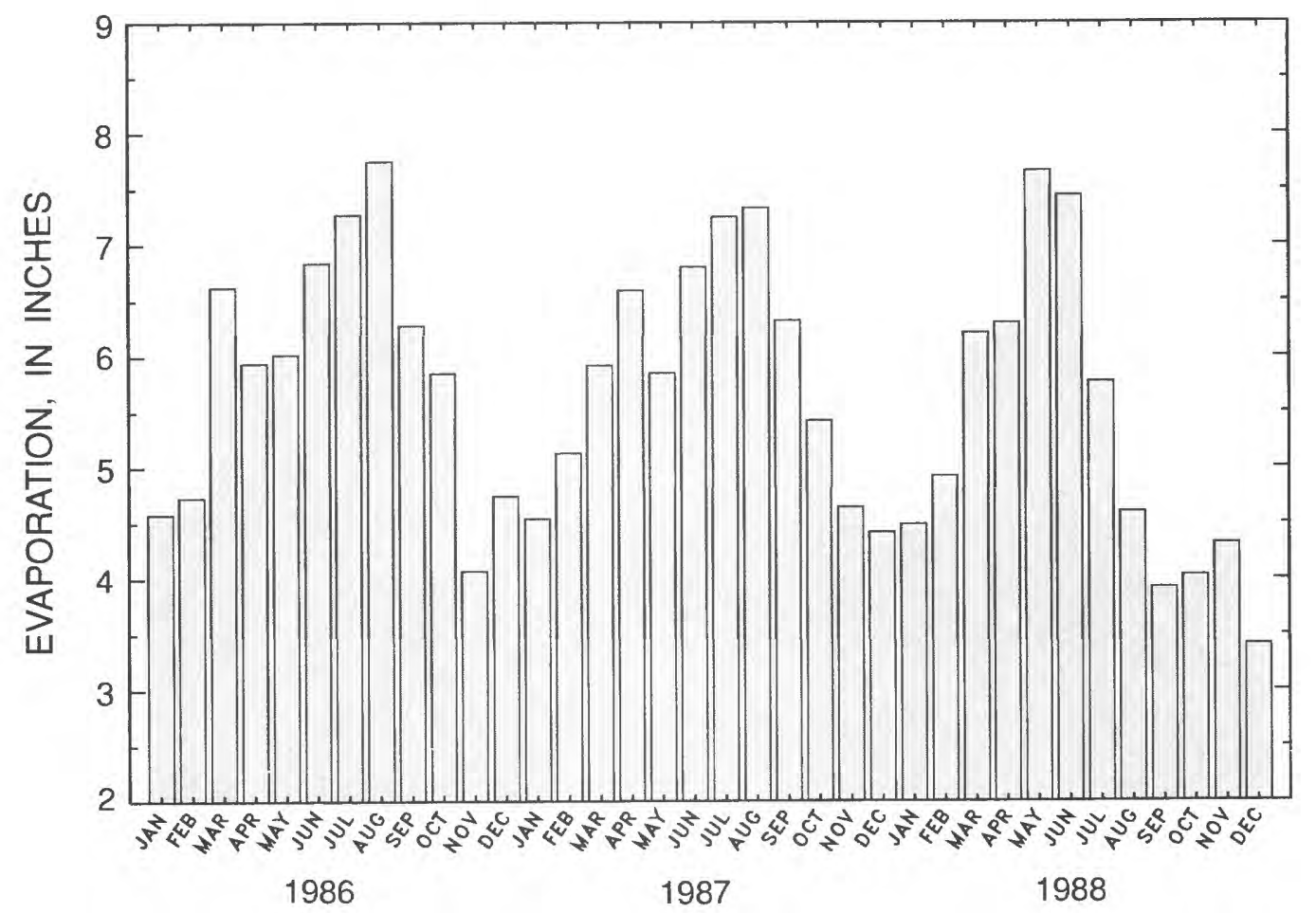

Figure 5.--Monthly pan evaporation at Gurabo Agricultural Experimental Station, 1986-1988. 
the valley. All of the streams and most of the creeks are perennial. A few creeks are ephemeral, particularly along the eastern boundary of the Gurabo-Juncos subarea.

The largest streams flowing through the valley are the Río Grande de Loíza, the Río Gurabo, the Río Valenciano, the Río Turabo, the Río Caguiitas, and the Río Bairoa. Streamflow hydrographs recorded by the USGS at sites on the Río Grande de Loíza, the Río Gurabo, the Río Turabo, and the Río Valenciano are shown in figure 6. Gaging stations on the Río Grande de Loíza at Caguas (station number 50055000) and the Río Gurabo at Gurabo (50057000) are located in the lower part of the valley, in an area where the stream is in direct connection with the alluvial aquifer. However, stations on the Río Turabo at Borinquen (50053050) and the Río Valenciano near Juncos (50056400) are located just upstream from the bedrock alluvium contact (fig. 7).

Dry and rainy seasons may be discerned by inspection of the hydrographs in figure 6. A simple statistical time series analysis of the streamflow based on the mean and the standard deviation parameters was performed to identify wet and dry periods (fig. 6). The average flows for the years 1985 through 1988 were $268,178,28$, and $69 \mathrm{ft}^{3} / \mathrm{s}$ in the Río Grande de Loíza, the Rín Gurabo, the Río Turabo, and the Río Valenciano, respectively (Curtis and others, 1989). Periods of below-average streamflow were most common from January through April. The analysis indicated two rainy or wet seasons, one during May and the other from September to November. In his study of the limnology of the Lago Loíza, Quiñones-Márquez (1980) identified the dry season as January to July and the rainy season as August to December.

Other important streams flowing through the Caguas subarea are the Río Bairoa and the Río Cagüitas. These streams are only measured periodically during low-flow studies or as part of the USGS island-wide water-quality monitoring program. These two streams are tributaries of the larger Río Grande de Loíza downstream of the Río Grande de Loíza gaging station (50055000) (fig. 7).

Annual and monthly flow duration curves have been developed by Quiñones-Márquez and others (1984) for streamflow gaging stations on the Río Grande de Loíza, the Río Gurabo, and the Río Valenciano (fig. 8). Flow duration curves provide a measure of the frequency of stream discharge. A discharge that is equalled or exceeded 90 percent of the time is considered representative of low-flow conditions. During low flow, a quasi-steady-state condition is approached, because the slope of the streamflow recession curve diminishes to nearly zero. The change in storage of the aquifer during low-flow conditions generally is small; that is, inflow minus outflow approaches zero. Steady-state conditions are rarely attainable in relatively small aquifers, such as this alluvial aquifer, because the main source of water (rainfall recharge) is highly variable and occurs throughout the entire year. Nonetheless, from the analysis of the flow duration curves in figure 8 , the discharge that was equalled or exceeded 90 percent of the time for the Río Grande de Loíza, the Río Gurabo, and the Río Valenciano was 40.0, 18.0, and $7.2 \mathrm{ft}^{3} / \mathrm{s}$, respectively. Therefore, discharges less than these were considered to represent quasi-steady-state conditions.

Estimates of 7-day, 10-year low flow at selected sites throughout the Island are presented in a report by Colón-Dieppa and Quiñones-Aponte (1985). This statistical parameter is defined as the discharge at a 10 -year recurrence interval taken from a frequency curve of annual values of the lowest mean discharge for seven consecutive days. Estimates of the 7-day, 10-year low flow help to establish the dependable flow of streams that is available for dilution of wastewater effluent and for water supply. In the study area, the 7-day, 10-year low flow was estimated at three gaged sites: the Río Grande de Loíza at Caguas $\left(14 \mathrm{ft}^{3} / \mathrm{s}\right)$, the Río Gurabo at Gurabo $\left(6.8 \mathrm{ft}^{3} / \mathrm{s}\right)$, and the Río Valenciano near Juncos $\left(3.3 \mathrm{ft}^{3} / \mathrm{s}\right)$. Estimates were also obtained for three ungaged sites: the Río Grande de Loíza at San Lorenzo $\left(6.8 \mathrm{ft}^{3} / \mathrm{s}\right)$, the Río Gurabo about 6 mi upstream from the gaging station $\left(2.8 \mathrm{ft}^{3} / \mathrm{s}\right)$, and the Río Gurabo just below Highway 943 at Gurabo $\left(6.9 \mathrm{ft}^{3} / \mathrm{s}\right)$. Although precipitation in the upper Río Grande de Loíza is considerable, long-term droughts do occur. The last significant drought in the basin was during 1976. The mean-annual discharges at the Río Grande de Loíza at Caguas and the Río Gurabo at Gurabo stations for 1976 were 148 and $78 \mathrm{ft}^{3} / \mathrm{s}$, respectively, compared to the mean discharge of 268 and $178 \mathrm{ft}^{3} / \mathrm{s}$ at the two stations for the years 1985 through 1988.

Two major floods have been recorded at the Río Grande de Loíza in the last 50 years: the flood of August 4, 1945 and that of September 6, 1960. The flood of September 6, 1960, reported by Fields (1972), was the highest measured discharge for the Río Grande de Loíza, the Río Gurabo, and their tributaries. Flood 
frequency analysis for selected sites on the Río Grande de Loiza and the Río Gurabo indicated that the recurrence interval of the September 6, 1960 flood at the Río Grande de Loíza at Caguas gaging station, which had a peak discharge of $71,500 \mathrm{ft}^{3} / \mathrm{s}$, was about 35 years. The peak discharge of $60,000 \mathrm{ft}^{3} / \mathrm{s}$ at the Río Gurabo at Gurabo gaging station also had a recurrence interval of about 35 years (Fields, 1972).

Although no major flood has occurred in the Caguas-Juncos Valley since 1960, concern about flooding has grown as a result of increased urban development in the valley. Of special importance is the city of Caguas, which has grown from a population of 95,661 in 1970 to 117,959 in 1980 and has a projected population of 158,113 in 2005 (Puerto Rico Planning Board, 1984). A consequence of urban development along the principal flood plains in the valley is the large percentage of paved areas that increase runoff, and therefore, the probability of floods.

The Lago Loíza, a man-made reservoir constructed in 1953 to provide public water to the San Juan metropolitan area, is located in the lower part of the valley (fig. 1). The main inflows to the Lago Loíza are the Río Grande de Loíza and the Río Gurabo. The capacity of Lago Loíza has been reduced by the enormous amount of sediments that have been transported to the reservoir by the streams. The reservoir's capacity has declined from 21,700 acre-ft in 1953 to about 10,000 acre-ft in 1985. Based on these capacities, the average rate of capacity depletion is estimated at about 1.8 percent annually. At this rate, the useable life of the reservoir is about 23 years (Quiñones and others, 1989).

\section{GROUND-WATER HYDROLOGY}

Ground water in the Caguas-Juncos Valley is an important part of the hydrologic cycle. Some human activities in the valley today depend on ground water, but as the population increases, the stress on the water resources will also increase. To quantitatively evaluate the potential for ground-water development in the valley, information is needed concerning wells and pumpage, the physical limits of the aquifer, hydraulic characteristics of the aquifer, the occurrence and movement of ground water, stream-aquifer relations, and ground-water storage.

\section{Water Wells and Use}

The saturated sands, gravels, silts, and clays of the Caguas-Juncos alluvium furnish water to 49 active wells scattered throughout the valley. A comprehensive inventory of these and other wells in the valley was conducted as part of this investigation. General information on each of the inventoried wells is provided in table 1. This table also contains information on about 15 observation wells drilled by the USGS during this investigation. The total number of active and inactive wells inventoried, including observation wells, was 119.

Most wells in the valley are screened in the alluvial deposits, which constitutes the principal aquifer in the area (fig. 7). The depth of wells in these deposits is variable. Well depths average $134 \mathrm{ft}$ and generally ( 67 percent) are between 38 and $230 \mathrm{ft}$. The few wells that have been completed in the bedrock formations generally yield less than $20 \mathrm{gal} / \mathrm{min}$.

Yields of wells in the Caguas-Juncos Valley are variable, but pumping rates up to $310 \mathrm{gal} / \mathrm{min}$ have been recorded. PRASA operates four pumping centers (Bairoa and Las Carolinas, in Caguas; Gurabo Agricultural Experimental Station in Gurabo; and Central Juncos in Juncos; table 1 and fig. 7) and a few other scattered wells of low yield (less than 50 $\mathrm{gal} / \mathrm{min}$ ). The public-supply wells at the Bairoa well battery (wells 41, 42, 43, 44, and 45) yielded about $140 \mathrm{gal} / \mathrm{min}$ per well, but these wells were inactivated by PRASA during 1985, because of contamination problems. In 1987, PRASA also inactivated wells 6 , 7 , and 8 at Las Carolinas. The yield of these wells averaged about $55 \mathrm{gal} / \mathrm{min}$ per well. PRASA wells in Gurabo (wells 51 and 52) and in Juncos (wells 56, 57. $58,59,60$, and 61 ) are still in use and yield about 275 and $270 \mathrm{gal} / \mathrm{min}$ per well, respectively. Ground-water withdrawal for public-water supply has been reduced from about $3.5 \mathrm{Mgal} / \mathrm{d}$ in 1986 to about $2.6 \mathrm{Mgal} / \mathrm{d}$ in 1988 (table 2).

Dairy farms throughout the valley use ground water in their day-to-day operations, but generally do not use it for potable water supply. Dairy wells commonly yield about $30 \mathrm{gal} / \mathrm{min}$ and are used mainly during daylight hours. A total of 22 active dairy wells have been identified throughout the valley (table 1). The estimated total ground-water pumpage at dairy farms averaged about $0.26 \mathrm{Mgal} / \mathrm{d}$ from 1986 to 1988 (table 2).

Another category of ground-water use in the valley is industrial use. There are eight active industrial wells in the valley with yields ranging from 17 to about $40 \mathrm{gal} / \mathrm{min}$. Total withdrawal from these wells averaged about $0.15 \mathrm{Mgal} / \mathrm{d}$ from 1986 to 1988 

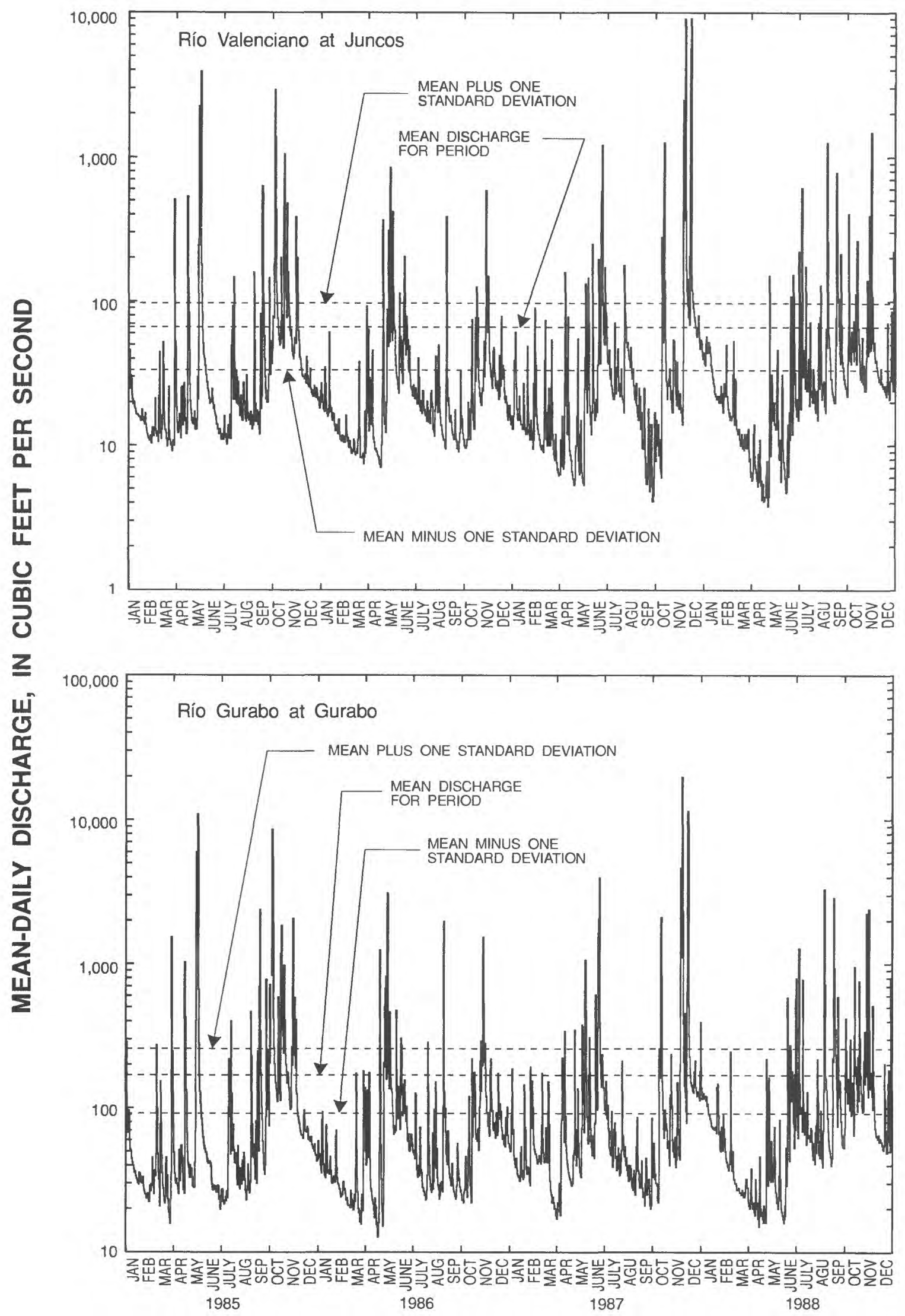

Figure 6a.--Streamflow hydrographs for Río Valenciano at Juncos (50053050) and Río Gurabo at Gurabo (50055000), 1985-1988. 

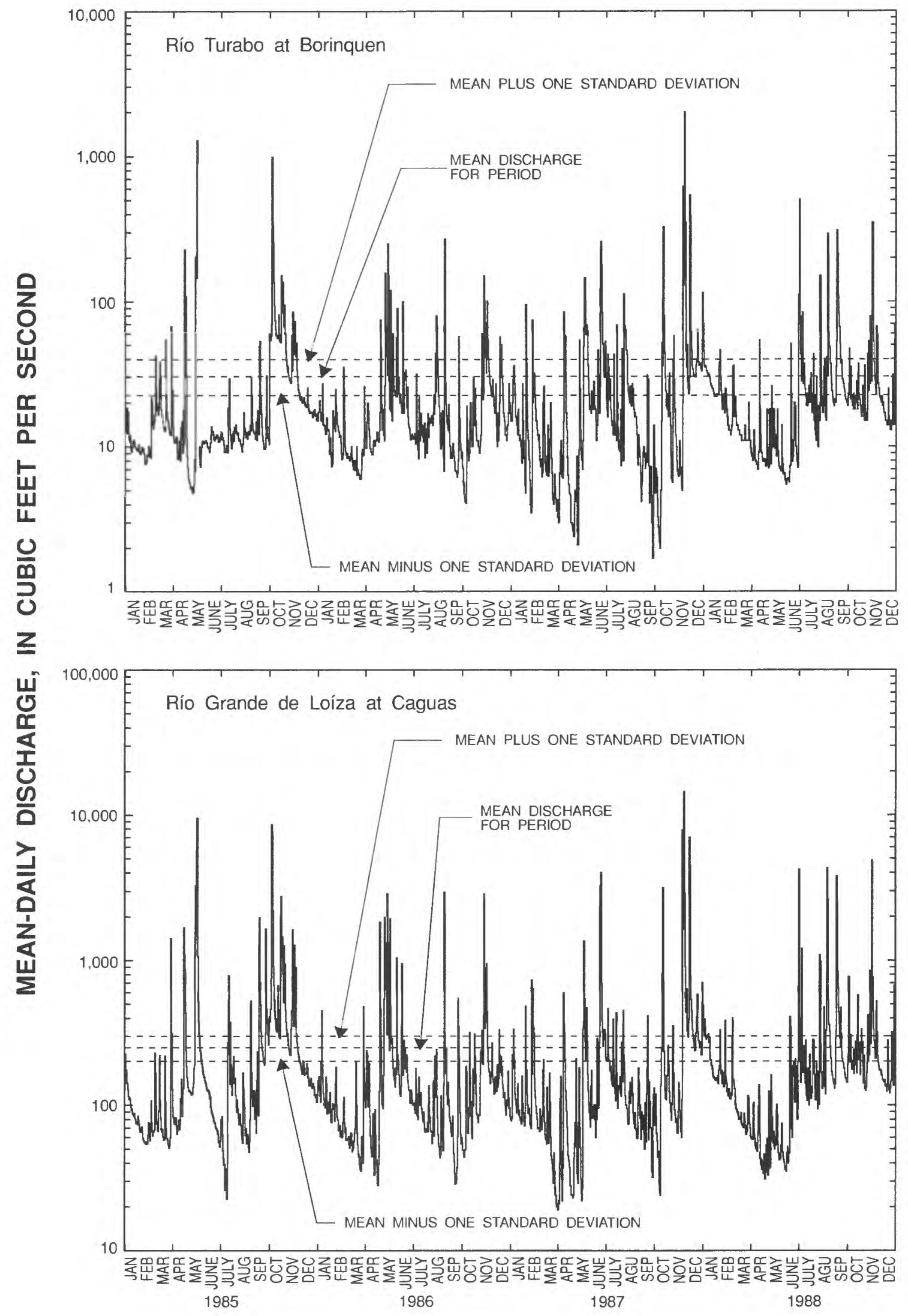

Figure 6b.--Streamflow hydrographs for Río Turabo at Borinquen (50053050) and Río Grande de Loíza at Caguas (50055000), 1985-1988. 


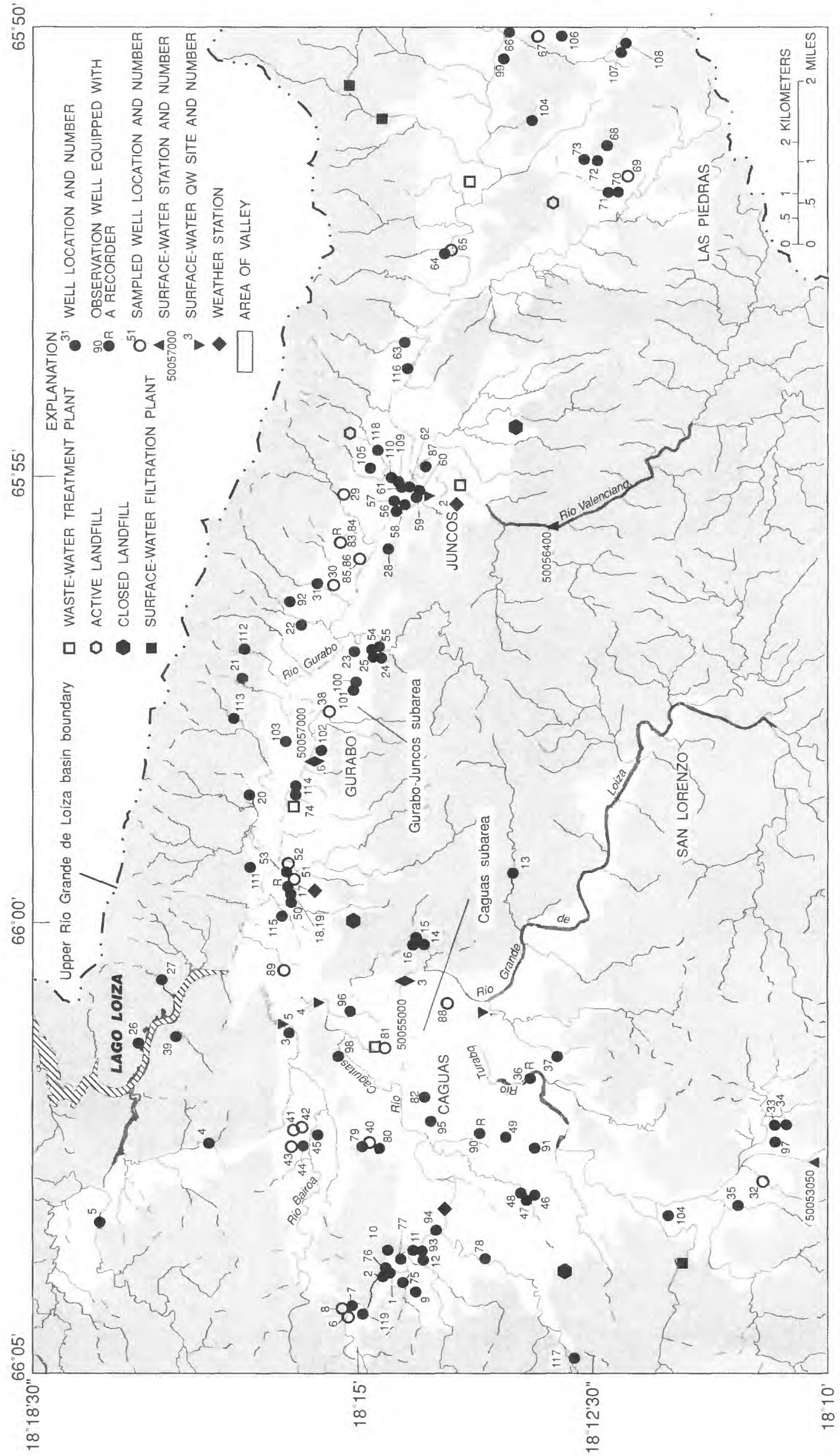

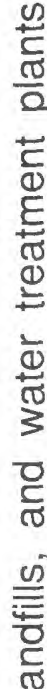

$\stackrel{\infty}{ \pm}$

응

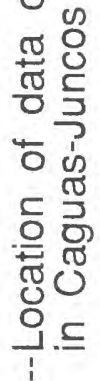

$N$

는 


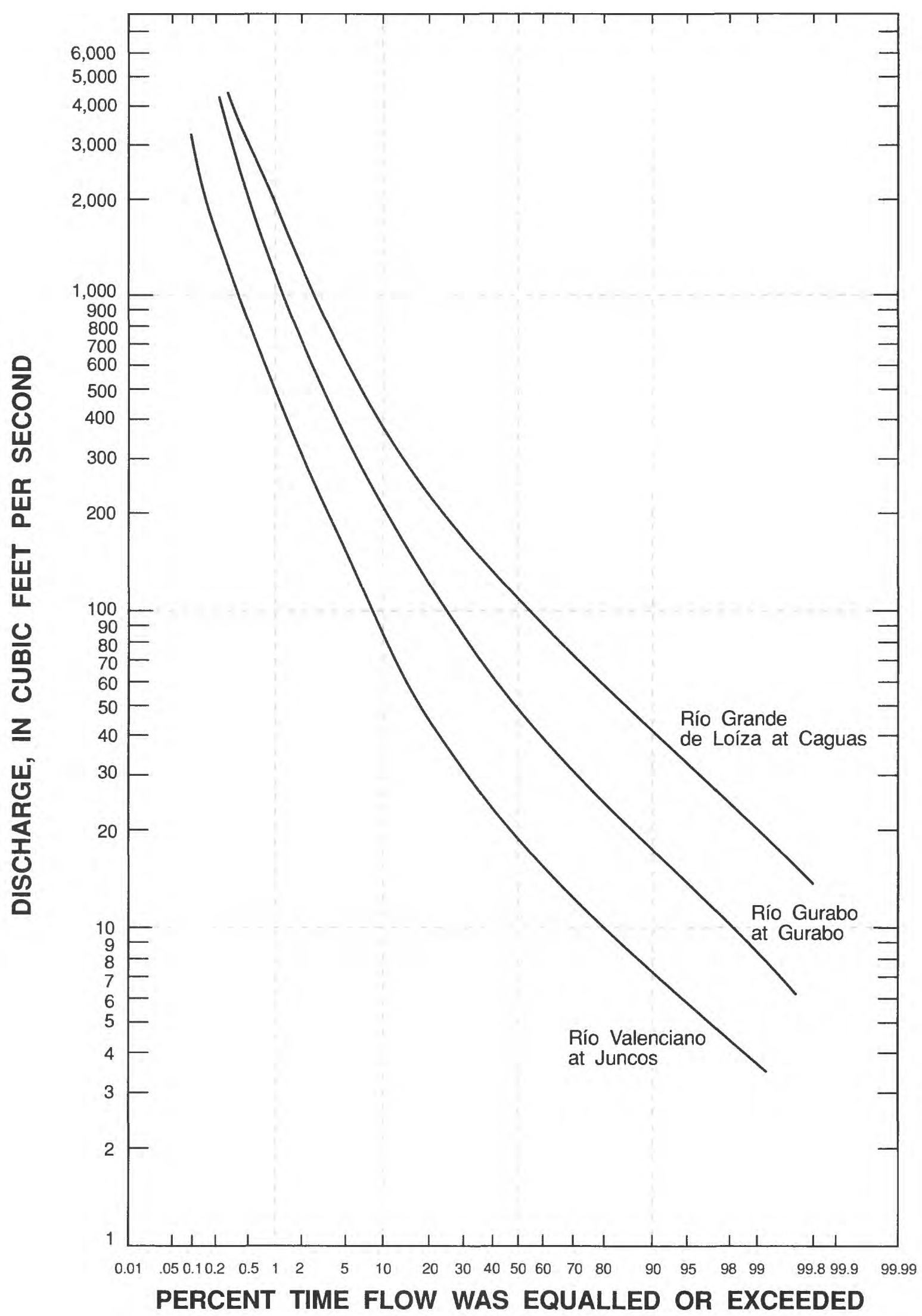

Figure 8.--Flow-duration curves for Río Grande de Loíza at Caguas, Río Gurabo at Gurabo, and Río Valenciano at Juncos. 
Table 1.--Description of wells inventoried in the Caguas-Juncos Valley

[leaders (--), no data]

\begin{tabular}{|c|c|c|c|c|c|c|}
\hline $\begin{array}{l}\text { Well number } \\
\text { and name }\end{array}$ & $\begin{array}{c}\text { Site } \\
\text { identification }\end{array}$ & $\begin{array}{l}\text { Well } \\
\text { depth } \\
\text { (feet) }\end{array}$ & status & $\begin{array}{c}\text { Yield } \\
\text { (gallons } \\
\text { per } \\
\text { minute) }\end{array}$ & $\begin{array}{c}\text { Depth to } \\
\text { bedrock } \\
\text { (feet) }\end{array}$ & Use of water \\
\hline 1. Cartagena 1 & 181448066034500 & 90 & active & 30 & -- & domestic/dairy \\
\hline 2. Cartagena 2 & 181444066035000 & 270 & active & 30 & 80 & domestic/dairy \\
\hline 3. Emilio López & 181544066011500 & 110 & active & 30 & $\ldots$ & domestic/dairy \\
\hline 5. Las Palmas & 181745066031000 & 110 & active & 17 & -- & industrial \\
\hline 6. Las Carolinas AAA 4A & 181508066041800 & 287 & inactive & 50 & 60 & public \\
\hline 7. Las Carolinas AAA $3 \mathrm{~A}$ & 181512066041100 & 350 & inactive & -- & 56 & public \\
\hline 8. Las Carolinas AAA B & 181508066041801 & - & inactive & 60 & 38 & public \\
\hline 9. Robinson 1 & 181428066040200 & 50 & inactive & -- & -- & domestic \\
\hline 10. Autopista & 181445066033600 & 47 & destroyed & -- & -- & - \\
\hline 11. Chevron Gas & 181427066033300 & 190 & active & 20 & -- & commercial \\
\hline 14. Dr. Quiñones 1 & 181422066001000 & 80 & destroyed & -- & -- & -- \\
\hline 15. Dr. Quiñones 2 & 181425066000400 & 70 & destroyed & -- & -- & -- \\
\hline 16. Dr. Quiñones 3 & 181427066000600 & -- & destroyed & -- & -- & -- \\
\hline 17. Gurabo 50 - USGS & 181550065593201 & 145 & abandoned & -- & -- & observation \\
\hline 18. UPR-EEA 1 & 181548065594000 & 125 & abandoned & -- & -- & -- \\
\hline 19. UPR-EEA 2 & 181548065593800 & 340 & abandoned & -- & -- & -- \\
\hline 20. Finca Toqui & 181615065573200 & & inactive & -- & -- & dairy \\
\hline 21. El Bambú AAA & 181622065571500 & 300 & active & -- & 10 & public \\
\hline 22. César Rivera & 181539065564300 & 130 & inactive & -- & $\ldots$ & dairy \\
\hline 23. Carlos Sánchez & 181503065565600 & 110 & active & 30 & -- & dairy \\
\hline 24. Johnson \& Johnson 3 & 181447065570200 & - & inactive & -- & -- & industrial \\
\hline 25. Johnson \& Johnson 2 & 181449065570200 & -- & active & 30 & -- & industrial \\
\hline 26. Juan Carrasquillo & 182119066041200 & -- & active & -- & -- & dosmestic \\
\hline 27. Jaguas AAA & 181708066003700 & -- & abandoned & -- & -- & public \\
\hline 28. Zoillo Méndez 1 & 181443065553700 & 80 & active & 20 & -- & dairy \\
\hline 29. Zoilo Méndez 2 & 181514065661100 & 250 & active & 30 & -- & dairy \\
\hline 31. Eneido Mendoza 2 & 181530065561000 & 150 & active & 20 & -- & dairy/domestic \\
\hline 32. Evaristo Delgado & 181046066024700 & -- & active & 20 & -- & dairy \\
\hline 33. Ubarri 1 & 181035066021100 & 75 & abandoned & -- & -- & dairy \\
\hline 34. Ubarri 2 & 181029066021500 & 60 & active & 20 & -- & dairy/domestic \\
\hline 35. Angel Zapata & 181058066030600 & 200 & inactive & -- & -- & domestic \\
\hline 36. Manrique 1 & 181311066013900 & 250 & inactive & -- & -- & observation \\
\hline 37. Manrique 2 & 181301066012200 & -- & active & 30 & -- & dairy \\
\hline 38. Pedro Morales & 181524065573900 & 40 & active & 20 & -- & industrial \\
\hline 39. Lake View AAA & 181659066012000 & 200 & active & 18 & 77 & public \\
\hline 40. Quality Elec. & 181454066022400 & 81 & active & 40 & -- & industrial \\
\hline 41. Bairoa AAA 9 & 181544066021500 & 140 & inactive & -- & $>118$ & public \\
\hline 42. Pozo Viejo AAA & 181538066021300 & 116 & inactive & 142 & 110 & public \\
\hline 43. Bairoa AAA 11 & 181547066022500 & 140 & inactive & 135 & 127 & public \\
\hline 44. Bairoa AAA 13 & 181538066022500 & 135 & abandoned & -- & -- & -- \\
\hline 45. Bairoa AAA 10 & 181528066021600 & 125 & inactive & -- & $>85$ & public \\
\hline 46. Hospital Regional 1 & 181314066025600 & 64 & inactive & -- & 60 & public \\
\hline 47. Hospital Regional 3 & 181318066025800 & 95 & inactive & -- & 87 & public \\
\hline 48. Hospital Regional 2 & 181318066025500 & 43 & abandoned & $-\infty$ & 40 & -- \\
\hline 49. R. Diez & 181332066022100 & 80 & active & 30 & - & commercial \\
\hline 50. Gurabo AAA 5 & 181550065594500 & 150 & abandoned & -- & 115 & -- \\
\hline 51. Gurabo AAA 6 & 181548065592900 & 123 & active & 240 & -- & public \\
\hline 52. Gurabo AAA 7 & 181549065592100 & 160 & active & 310 & -- & public \\
\hline 53. Gurabo AAA 8 & 181550065592700 & 145 & abandoned & -- & 140 & - \\
\hline 54. Janssen 1 & 175655065565500 & 90 & active & 20 & 50 & industrial \\
\hline 55. Janssen 2 & 175649065565400 & 90 & inactive & -- & 50 & industrial \\
\hline 56. Juncos AAA 1 & 181437065552200 & 140 & active & 300 & 100 & public \\
\hline 57. Juncos AAA 2 & 181437065551900 & 120 & active & 300 & 115 & public \\
\hline 58. Juncos AAA 7 & 181432065550500 & 90 & active & 250 & 93 & public \\
\hline 59. Juncos AAA 4 & 181424065550700 & 110 & active & 275 & 102 & public \\
\hline 60. Juncos AAA 5 & 181424065551400 & 88 & active & 250 & -- & public \\
\hline
\end{tabular}


Table 1.--Description of wells inventoried in the Caguas-Juncos Valley--Continued [leaders (--), no data]

\begin{tabular}{|c|c|c|c|c|c|c|}
\hline $\begin{array}{l}\text { Well number } \\
\text { and name }\end{array}$ & $\begin{array}{c}\text { Site } \\
\text { identification }\end{array}$ & $\begin{array}{l}\text { Well } \\
\text { depth } \\
\text { (feet) }\end{array}$ & Status & $\begin{array}{l}\text { Yield } \\
\text { (gallons } \\
\text { per } \\
\text { minute) }\end{array}$ & $\begin{array}{c}\text { Depth to } \\
\text { bedrock } \\
\text { (feet) }\end{array}$ & Use of water \\
\hline 61. Juncos AAA 3 & 181432065551400 & 90 & active & 250 & 88 & public \\
\hline 62. FESA Juncos 3 & 181429065550600 & 117 & abandoned & - & 93 & - \\
\hline 63. Cardona & 181435065533000 & 75 & abandoned & -- & -- & -- \\
\hline 65. Eduardo Berríos 2 & 181402065523200 & 60 & active & 30 & -- & dairy \\
\hline 66. Boquerón AAA 1 & 181322065500300 & 180 & abandoned & -- & -- & - \\
\hline 67. Boquerón AAA 2 & 181309065501300 & 98 & active & 80 & -- & public \\
\hline 68. Hermosura 1 & 181219065512400 & -- & active & 20 & -- & dairy \\
\hline 69. Hermosura 2 & 181206065514400 & -- & active & 20 & -- & dairy \\
\hline 70. Hermosura 3 & 181213065515700 & -- & inactive & -- & -- & dairy \\
\hline 71. Hermosura 4 & 181218065515300 & 55 & abandoned & -- & -- & -- \\
\hline 73. Hermosura 6 & 181234065513200 & 83 & active & 21 & -- & dairy \\
\hline 74. Oscar Dávila & 181544065583200 & 52 & inactive & - & -- & domestic \\
\hline 75. Robinson 2 & 181430066040100 & 17 & inactive & -- & -- & dairy \\
\hline 76. Cartagena 3 & 181449066034500 & 75 & inactive & -- & -- & dairy \\
\hline 77. Robinson 3 & 181438066034200 & -- & active & 10 & -- & domestic \\
\hline 78. Vicente Santos & 181346066033700 & 70 & active & 10 & -- & domestic \\
\hline 79. Old Savoy & 181459066022600 & 66 & abandoned & - & -- & industrial \\
\hline 80. Century Packing & 181452066022500 & 81 & active & 32 & -- & industrial \\
\hline 81. $\mathrm{CJ}-\mathrm{TW} 1$ & 181445066011500 & 32.5 & inactive & -- & 33 & observation \\
\hline 82. $\mathrm{CJ}-\mathrm{TW} 2$ & 181422066015300 & 45 & inactive & -- & 45 & observation \\
\hline 83. $\mathrm{CJ}-\mathrm{TW} 3 \mathrm{~A}$ & 181513065554600 & 87.5 & inactive & -- & 99 & observation \\
\hline 84. CJ-TW $3 B$ & 181513065554601 & 38 & inactive & -- & 38 & observation \\
\hline 85. CJ-TW4A & 181501065555500 & 34.5 & inactive & -- & 39 & observation \\
\hline 86. $\mathrm{CJ}-\mathrm{TW} 4 \mathrm{~B}$ & 181501065555501 & 144 & inactive & -- & 160 & observation \\
\hline 87. CJ-TW6 & 181415065545400 & 102 & inactive & -- & 102 & observation \\
\hline 88. $\mathrm{CJ}-\mathrm{TW} 7$ & 181406066004600 & 76 & inactive & $\ldots$ & 79 & observation \\
\hline 91. CJ-TW11 & 181311066022500 & 110 & inactive & -- & 117 & observation \\
\hline 92. César Rivera 2 & 181548065562600 & $>495$ & abandoned & -- & - & -- \\
\hline 93. Luis ortiz 2 & 181422066033300 & 49 & inactive & -- & -- & dairy \\
\hline 94. Ernesto Jiménez & 181410066031300 & 50 & active & 10 & -- & domestic \\
\hline 95. Hosp. San Rafael & 181418066020600 & -- & active & 36 & -- & industrial \\
\hline 96. Caguas Sugar Growers & 181511066005700 & -- & active & 18 & -- & industrial \\
\hline 97. Villa Borinquen & 181041066022500 & -- & abandoned & -- & -- & - \\
\hline 98. Hwy. 30 & 181514066012700 & -- & abandoned & -- & -- & -- \\
\hline 99. Pedro Ochoa & 181325065502300 & -- & active & -- & -- & dairy \\
\hline 100. Berríos 1 & 181504065571700 & -- & active & -- & -- & domestic \\
\hline 101. Berrios 2 & 181504065571701 & -- & active & -- & -- & domestic \\
\hline 102. Jardines de Gurabo & 181529065580500 & 130 & abandoned & $\ldots$ & -- & -- \\
\hline 103. Buenavista & 181550065575700 & - & active & -- & -- & dairy \\
\hline 104. Tropigas & 181158066030500 & 40 & inactive & -- & -- & industrial \\
\hline 105. Finca Batey & 181453065545400 & $\ldots$ & active & -- & -- & dairy \\
\hline 106. Hacienda Cuco 1 & 181250065501200 & -- & active & 20 & -- & dairy \\
\hline 107. Hacienda Cuco 2 & 181219065502000 & -- & active & 20 & -- & dairy \\
\hline 108. Hacienda Cuco 3 & 181220065502200 & -- & active & 20 & -- & dairy \\
\hline 109. FESA Juncos 1 & 181438065550500 & 150 & abandoned & -- & 75 & -- \\
\hline 110. FESA Juncos 4 & 181441065550400 & 90 & abandoned & -- & -- & -- \\
\hline 111. Quintero & 181613065592000 & -- & abandoned & -- & -- & -- \\
\hline 112. Hato Nuevo $A A A$ & 181618065565900 & 300 & abandoned & -- & 20 & public \\
\hline 113. Celada AAA & 181620065574400 & 400 & abandoned & -- & 60 & public \\
\hline 114. Celada (F. Cueros) & 181544065582700 & 360 & abandoned & -- & 0 & public \\
\hline 115. Gurabo AAA & 181552065595100 & 140 & abandoned & -- & 122 & public \\
\hline 116. Placita AAA & 181430065535200 & 100 & destroyed & -- & 89 & public \\
\hline 117. Canaboncito $A A A$ & 181247066044600 & 230 & inactive & -- & 60 & public \\
\hline 118. CJ-TW 16 & 181448065544200 & 95 & inactive & -- & 130 & observation \\
\hline 119. Las Carolinas AAA 2A & 191510066042100 & 360 & abandoned & -- & 50 & public \\
\hline
\end{tabular}


Table 2.--Ground-water withdrawals from the alluvial aquifer in the Caguas-Juncos Valley, 1986, 1987, and 1988

\begin{tabular}{|c|c|c|c|c|}
\hline Well number and name & $\begin{array}{l}\text { Yield } \\
\text { (gallons per } \\
\text { minute) }\end{array}$ & \multicolumn{3}{|c|}{$\begin{array}{c}\text { Withdrawals by year } \\
\text { (million gallons per day) } \\
1986 \\
1987\end{array}$} \\
\hline \multicolumn{5}{|l|}{ DAIRY WELLS } \\
\hline $\begin{array}{l}\text { 1. Cartagena } 1 \\
\text { 2. Cartagena } 2 \\
\text { 3. Emilio López } \\
\text { 23. Carlos Sánchez } \\
\text { 28. Zoilo Méndez } 1\end{array}$ & $\begin{array}{l}30 \\
30 \\
30 \\
30 \\
20\end{array}$ & $\begin{array}{r}0.015 \\
.015 \\
.015 \\
.021 \\
.001\end{array}$ & $\begin{array}{r}0.015 \\
.015 \\
.015 \\
.021 \\
.001\end{array}$ & $\begin{array}{l}0.015 \\
.0 \\
.015 \\
.021 \\
.001\end{array}$ \\
\hline $\begin{array}{l}\text { 29. Zoilo Méndez } 2 \\
\text { 30. Eneido Mendoza } 1 \\
\text { 31. Eneido Mendoza } 2 \\
\text { 32. Evaristo Delgado } \\
\text { 34. Ubarri } 2\end{array}$ & $\begin{array}{l}30 \\
20 \\
20 \\
20 \\
20\end{array}$ & $\begin{array}{l}.015 \\
.007 \\
.010 \\
.010 \\
.012\end{array}$ & $\begin{array}{l}.015 \\
.007 \\
.010 \\
.010 \\
.012\end{array}$ & $\begin{array}{l}.015 \\
.007 \\
.010 \\
.010 \\
.012\end{array}$ \\
\hline $\begin{array}{l}\text { 37. Manrique } 2 \\
\text { 64. Eduardo Berríos } 1 \\
\text { 65. Eduardo Berríos } 2 \\
\text { 68. Hermosura } 1 \\
\text { 69. Hermosura } 2\end{array}$ & $\begin{array}{l}30 \\
30 \\
30 \\
20 \\
20\end{array}$ & $\begin{array}{l}.015 \\
.015 \\
.015 \\
.010 \\
.019\end{array}$ & $\begin{array}{l}.015 \\
.015 \\
.015 \\
.010 \\
.019\end{array}$ & $\begin{array}{l}.015 \\
.015 \\
.015 \\
.010 \\
.019\end{array}$ \\
\hline $\begin{array}{l}\text { 73. Hermosura } 6 \\
\text { 99. Pedro Ochoa } \\
\text { 103. Buenavista } \\
\text { 105. Finca Batey } \\
\text { 106. Hacienda Cuco } 1\end{array}$ & $\begin{array}{l}21 \\
20 \\
30 \\
25 \\
20\end{array}$ & $\begin{array}{l}.010 \\
.0 \\
.015 \\
.012 \\
.010\end{array}$ & $\begin{array}{l}.010 \\
.0 \\
.015 \\
.012 \\
.010\end{array}$ & $\begin{array}{l}.010 \\
.010 \\
.015 \\
.012 \\
.010\end{array}$ \\
\hline $\begin{array}{l}\text { 107. Hacienda Cuco } 2 \\
\text { 108. Hacienda Cuco } 3\end{array}$ & $\begin{array}{l}20 \\
20\end{array}$ & $\begin{array}{l}.010 \\
.010\end{array}$ & $\begin{array}{l}.010 \\
.010\end{array}$ & $\begin{array}{l}.010 \\
.010\end{array}$ \\
\hline & & 0.262 & 0.262 & 0.257 \\
\hline
\end{tabular}

INDUSTRIAL WELLS

5. Las Palmas

25. Johnson \& Johnson 2

38. Pedro Morales

40. Quality Elect.

54. Janssen 1

80. Century Packing

95. Hosp. San Rafael

96. Caguas Sugar Growers

1
3
2
4

Subtotal $=$

PUBLIC WELLS

50
50
60
25
18

240
310
300
300
250

0.024

.022

.010

.0

.014

.015

.035

.007

0.127

0.165

0.165

0.024

.022

.010

.038

.014

.015

.035

.007

.035

.007
0.0

.072

.088

.036

.026

0.0

.072

.088

.036

.026

.346

.446

.432

.432

.360
.0

.0

.026

.346

.446

.432

.432

.360 
Table 2.--Ground-water withdrawals from the alluvial aquifer in the Caguas-Juncos Valley, 1986, 1987, and 1988--Continued

\begin{tabular}{|c|c|c|c|c|c|}
\hline Well number and name & & $\begin{array}{c}\text { Yield } \\
\text { (gallons per } \\
\text { minute) }\end{array}$ & $\begin{array}{l}\text { Withdr } \\
\text { (million } \\
1986\end{array}$ & $\begin{array}{l}\text { rawals by yed } \\
\text { gallons per } \\
1987\end{array}$ & $\begin{array}{l}\text { day) } \\
1988\end{array}$ \\
\hline \multirow[t]{2}{*}{$\begin{array}{l}\text { 59. Juncos AAA } 4 \\
\text { 60. Juncos AAA } 5 \\
\text { 61. Juncos AAA } 3 \\
\text { 67. Boquerón AAA } 2\end{array}$} & & $\begin{array}{r}275 \\
250 \\
250 \\
80\end{array}$ & $\begin{array}{l}.396 \\
.360 \\
.360 \\
.115\end{array}$ & $\begin{array}{l}.396 \\
.360 \\
.360 \\
.115\end{array}$ & $\begin{array}{l}.0 \\
.360 \\
.0 \\
.115\end{array}$ \\
\hline & Subtotal & $=$ & 3.541 & 3.469 & 2.553 \\
\hline \multicolumn{6}{|c|}{ DOMESTIC/COMMERCIAL WELLS } \\
\hline $\begin{array}{l}\text { 11. Chevron Gas } \\
\text { 22. César Rivera } \\
\text { 26. Juan Carrasquillo } \\
\text { 49. R. Diez } \\
\text { 77. Robinson } 3\end{array}$ & & $\begin{array}{l}20 \\
20 \\
10 \\
30 \\
10\end{array}$ & $\begin{array}{r}0.010 \\
.002 \\
.004 \\
.015 \\
.002\end{array}$ & $\begin{array}{r}0.010 \\
.002 \\
.005 \\
.015 \\
.002\end{array}$ & $\begin{array}{l}0.010 \\
.0 \\
.004 \\
.015 \\
.002\end{array}$ \\
\hline \multirow[t]{3}{*}{$\begin{array}{l}\text { 78. Vicente Santos } \\
\text { 94. Ernesto Jiménez } \\
\text { 100. Berrios } 1 \\
\text { 101. Berrios } 2\end{array}$} & & $\begin{array}{l}10 \\
10 \\
30 \\
30\end{array}$ & $\begin{array}{l}.002 \\
.007 \\
.010 \\
.010\end{array}$ & $\begin{array}{l}.002 \\
.007 \\
.010 \\
.010\end{array}$ & $\begin{array}{l}.0 \\
.007 \\
.010 \\
.010\end{array}$ \\
\hline & Subtotal & $=$ & 0.062 & 0.063 & 0.058 \\
\hline & Total & $=$ & 3.992 & 3.959 & 3.033 \\
\hline
\end{tabular}

(table 2). Withdrawals from domestic and commercial wells for the same period averaged about $0.06 \mathrm{Mgal} / \mathrm{d}$. The total ground-water withdrawal for all water use categories was about $4 \mathrm{Mgal} / \mathrm{d}$ in 1986 and 1987, but decreased to $3 \mathrm{Mgal} / \mathrm{d}$ in 1988 (table 2).

Water-use data from PRASA indicated that the source of water for public supply within the upper Río Grande de Loíza basin are local surface water (10.6 $\mathrm{Mgal} / \mathrm{d}$ ), local ground water (3.5 Mgal/d), and imported water from outside the basin (4.5 Mgal/d from Guaynabo and $2.8 \mathrm{Mgal} / \mathrm{d}$ from Humacao). Ground water constituted about 16 percent of the total publicwater supply (about $21 \mathrm{Mgal} / \mathrm{d}$ ) in the area.

\section{Alluvial Aquifer}

The aquifer, which underlies the Caguas-Juncos Valley, consists of alluvial deposits (mostly gravel, sand, silt, and clay). Based on drillers logs and surface electrical resistivity surveys conducted during this investigation, the thickness of these alluvial deposits is variable, but averages about $80 \mathrm{ft}$ (fig. 9). The inactive Bairoa wells (wells 41, 42, 43, 44, and 45, in figure 7) are located in an area where the thickness of the alluvial deposits ranges from 85 to $127 \mathrm{ft}$. Apparently, the thickness of alluvial deposits in the Caguas subarea increases southwest of well 82 , and decreases to the north. The areas of greatest alluvial thickness are not necessarily the most productive, because aquifer productivity also depends on the hydraulic conductivity and depth to water. A section drawn for the Caguas subarea, from southwest to northeast (fig. 10), depicts layers of the different aquifer materials and the inferred location of the alluvium/bedrock contact.

The composition and thickness of the alluvial aquifer in the Gurabo-Juncos subarea is quite different from that in the Caguas subarea (fig. 10). In the Gurabo-Juncos subarea, the principal stream flows through the center of the valley creating the thicker lens along this axis. The aquifer thins toward the western and eastern ends of the valley and toward the northern and southern edges of the valley. In general, the mantle of alluvial deposits is thicker over a wider area in Gurabo and Juncos than in Caguas. Depths to bedrock average $126 \mathrm{ft}$ near the PRASA well battery in Gurabo (wells 50, 53, and 115), and $100 \mathrm{ft}$ in Juncos (wells 56, 57, 58, 59, 61, 62, and 109). 
Maximum thickness of the deposits in this area is about $160 \mathrm{ft}$ near Juncos (well 86) and $140 \mathrm{ft}$ near Gurabo (well 53; fig. 7).

From the interpretation and comparison of the representative cross-sections in figure 10 , the composition of the alluvial deposits seems to be dominated by clay and silt in Caguas and by sand and gravel in Gurabo-Juncos. The difference in the composition and thickness of the alluvial aquifer in both areas is related to their respective structural and depositional origins.

\section{Aquifer Hydraulic Characteristics}

The potential for ground-water development is directly related to the aquifer's hydraulic characteristics, namely, the transmissivity and the storage coefficient. Transmissivity is defined as the rate at which water is transmitted through a unit width of the aquifer under a unit hydraulic gradient (Lohman and others, 1972). These parameters are used in ground-water flow equations to evaluate how the aquifer system transmits and releases water to wells.

Transmissivity values in the alluvial aquifer were estimated from specific capacity data obtained from PRASA and from private well owners, and slug-tests. The specific capacity (discharge/drawdown) data is expressed as the well discharge per foot of drawdown at the pumping well after a specific time. The method used to estimate transmissivity values from the specific capacity data is described in detail by Theis and others (1963). Basically, this method solves the following equation for unconfined conditions:

$$
\mathrm{T}^{\prime}=\mathrm{Q} / \mathrm{s}\left(\mathrm{K}-264 \log _{10} 5 \mathrm{~S}+264 \log _{10} \mathrm{t}\right),
$$

where

$\mathrm{T}^{\prime}$ is transmissivity ((gal/min)/ft),

$\mathrm{Q}$ is pumping rate (gal/min),

$\mathrm{S}$ is total drawdown in the pumping well (ft),

$\mathrm{K}$ is an empirical constant which is a function of $\mathrm{r}$,

$r$ is the well radius ( $\mathrm{ft}$ ),

$S$ is storage coefficient,

$\mathrm{t}$ is total pumping time (days).

The following equation is then solved by iteration:

where

$$
\mathrm{T}=\mathrm{T}^{\prime}-(264 \mathrm{Q} / \mathrm{s}) \log _{10}\left(\mathrm{~T} 10^{-5}\right),
$$

$\mathrm{T}$ is computed transmissivity $((\mathrm{gal} / \mathrm{min}) / \mathrm{ft})$.
The transmissivity value computed by this method is representative of the length of screen open to the saturated thickness of the aquifer, and is converted to $\mathrm{ft}^{2} / \mathrm{d}$ units when multiplied by the corresponding conversion factors. A better estimate of the transmissivity value is found by computing hydraulic conductivity and then multiplying it by the entire aquifer thickness.

The slug-test is a method used to estimate the hydraulic conductivity and transmissivity from data obtained by measuring water level change inside a well when a predetermined volume or "slug" of water is suddenly removed from or injected into the well (Bouwer and Rice, 1976). The hydraulic conductivity can be computed by matching theoretical curves with field data. Then, the hydraulic conductivity is multiplied by the saturated thickness to obtain the transmissivity.

Specific-capacity data from 29 wells were analyzed to estimate transmissivity values, and slug tests were conducted at four piezometers. Transmissivity values estimated from specific-capacity data are shown in table 3 and those derived from slug tests are shown in table 4. Estimates of apparent transmissivity ranged from a low of $66 \mathrm{ft}^{2} / \mathrm{d}$ in well 66 (Boquerón AAA 1) located at the eastern boundary of the Gurabo-Juncos subarea, to a high of $4,770 \mathrm{ft}^{2} / \mathrm{d}$ in well 53 (Gurabo AAA 8), one of the PRASA wells at the western edge of the Gurabo-Juncos subarea. The average transmissivity was $1,350 \mathrm{ft}^{2} / \mathrm{d}$, and 67 percent of the values were within the range of 77 to 2,630 $\mathrm{ft}^{2} / \mathrm{d}$. Estimated values of transmissivity generally were higher in the Gurabo-Juncos subarea than in the Caguas subarea. The alluvial deposits contain a greater percentage of relatively permeable sand and gravel in the Gurabo-Juncos subarea as opposed to the Caguas subarea, where the alluvial deposits contain a large percentage of low permeability silt and clay.

A transmissivity distribution map, constructed from the estimated values (fig. 11), indicates higher transmissivities along the main stream channels. The Bairoa zone, near wells 41, 42, 43, 44, and 45 (fig. 7), appears to be the most productive (highest transmissivities) in the Caguas subarea. Within the Gurabo-Juncos subarea, larger transmissivity values occur in the areas near the Gurabo PRASA wells, near the Juncos PRASA wells, and along the Río Gurabo downstream from the confluence of the Río Valenciano and the Río Gurabo. High transmissivity values in the Caguas and Gurabo-Juncos subareas are associated with large saturated thicknesses of the aquifer. 


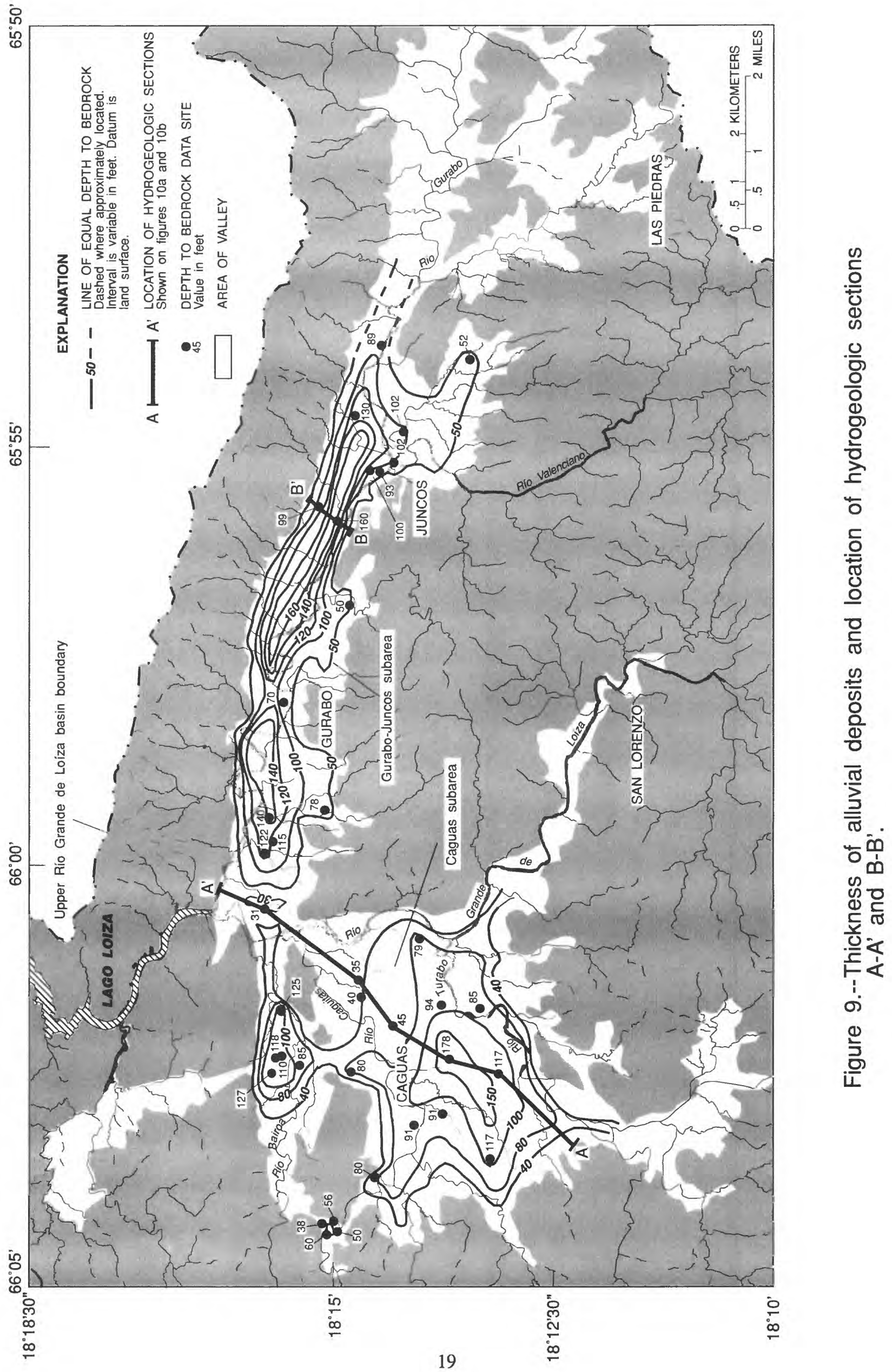




\section{EXPLANATION}

\begin{tabular}{|c|c|}
\hline & MOSTLY CLAYEY \\
\hline & MOSTLY GRAVEL \\
\hline & SAND AND SILT \\
\hline & WEATHERED ROCK? \\
\hline & BEDROCK \\
\hline & WATER TABLE \\
\hline & WELL AND NUMBER \\
\hline
\end{tabular}

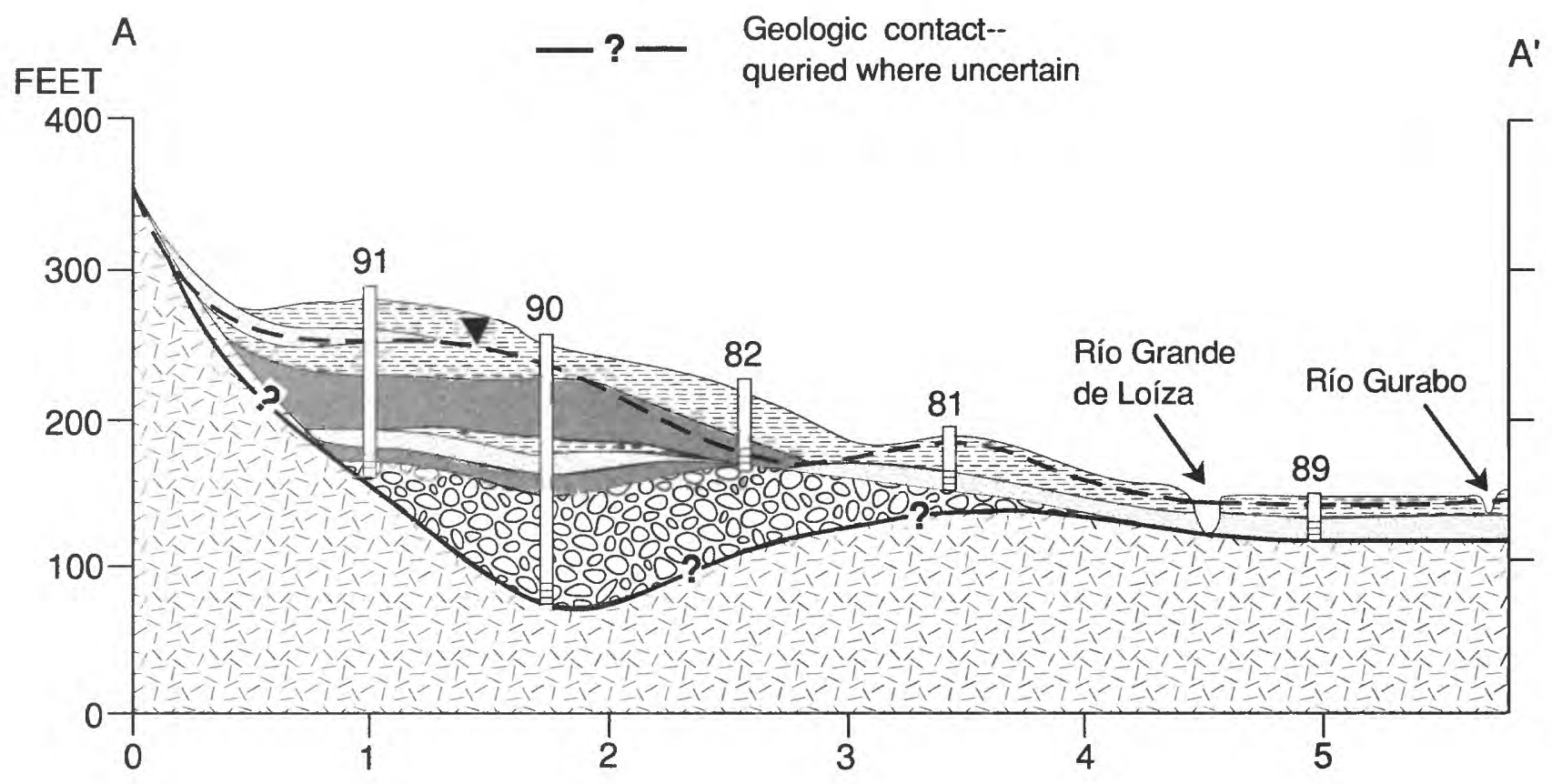

DISTANCE, IN MILES

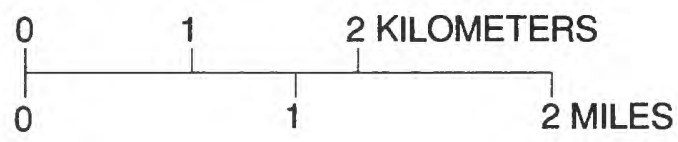

Figure 10a.--Hydrogeologic section A-A' along Caguas subarea (refer to figure 9 for location). 


\section{EXPLANATION}

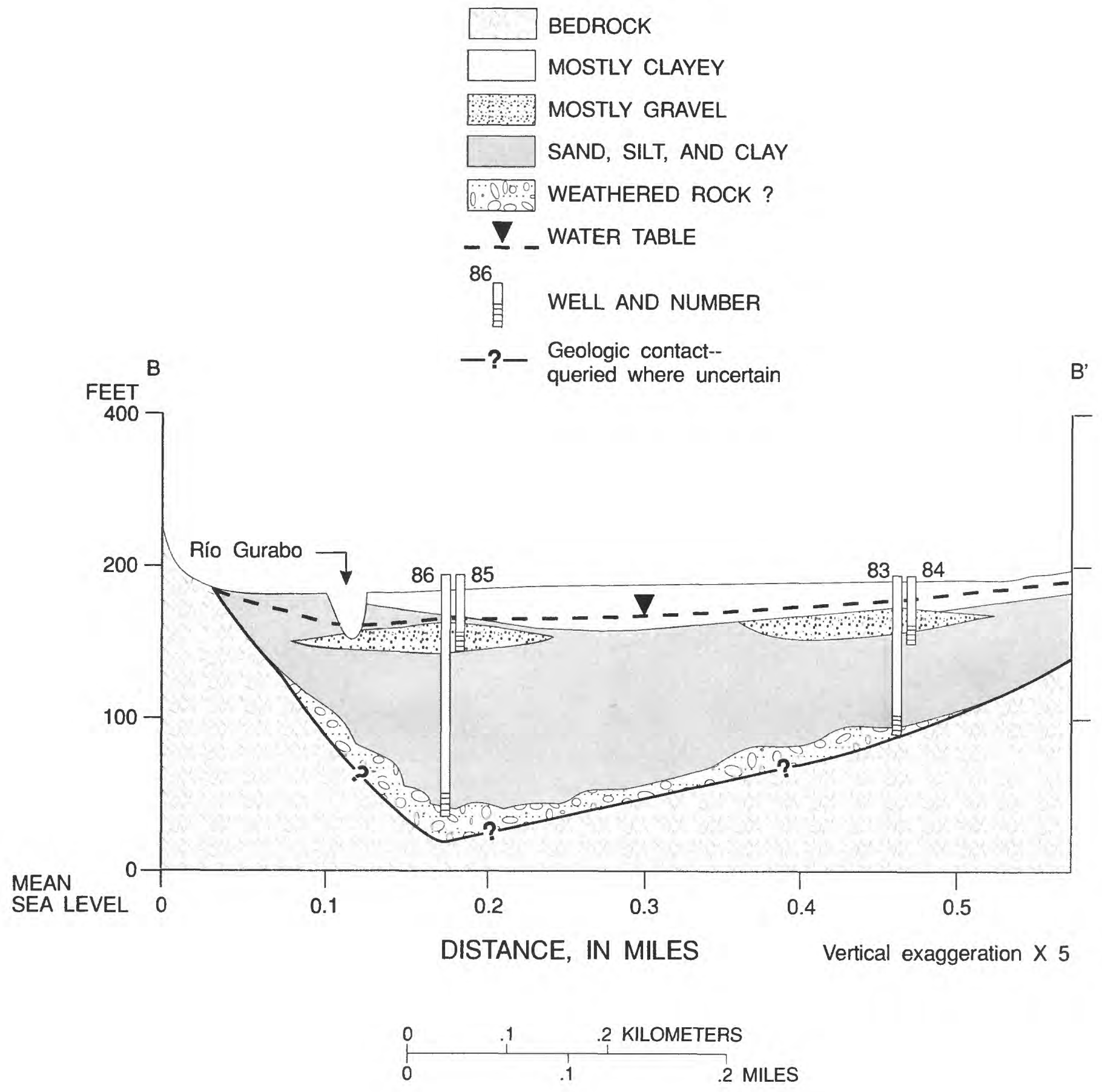

Figure 10b.--Hydrogeologic section B-B' along Gurabo-Juncos subarea (refer to figure 9 for location). 
Table 3. --Transmissivity estimates from specific-capacity data in the Caguas-Juncos Valley [gal/min, gallons per minute; ft, feet; ft/d, feet per day; $\mathrm{ft}^{2} / \mathrm{d}$, feet squared per day]

\begin{tabular}{|c|c|c|c|c|c|c|c|c|}
\hline $\begin{array}{c}\text { Well } \\
\text { number }\end{array}$ & $\begin{array}{l}\text { Pumping } \\
\text { rate } \\
\text { (gal/min) }\end{array}$ & $\begin{array}{c}\text { Drawdown } \\
\text { in well } \\
\text { (feet) }\end{array}$ & $\begin{array}{l}\text { Pumping } \\
\text { time } \\
\text { (days) }\end{array}$ & $\begin{array}{l}\text { Calculated } \\
\text { transmissivity } \\
\text { from specific } \\
\text { capacity data } \\
\left(\mathrm{ft}^{2} / \mathrm{d}\right)\end{array}$ & $\begin{array}{l}\text { Aquifers } \\
\text { saturated } \\
\text { thickness } \\
\text { (ft) }\end{array}$ & $\begin{array}{l}\text { Screen } \\
\text { length } \\
(\mathrm{ft})\end{array}$ & $\begin{array}{l}\text { Hydraulic } \\
\text { conductivity } \\
(\mathrm{ft} / \mathrm{d})\end{array}$ & $\begin{array}{l}\text { Apparent } \\
\text { transmissivity } \\
\left.\text { (ft } \mathrm{t}^{2} / \mathrm{d}\right)\end{array}$ \\
\hline $\begin{array}{r}65 \\
30 \\
3 \\
23 \\
2\end{array}$ & $\begin{array}{l}30.0 \\
30.0 \\
55.0 \\
30.0 \\
30.0\end{array}$ & $\begin{array}{r}23.0 \\
6.0 \\
9.0 \\
14.0 \\
8.0\end{array}$ & $\begin{array}{l}0.3 \\
0.3 \\
0.3 \\
0.3 \\
0.3\end{array}$ & $\begin{array}{r}201.4 \\
887.1 \\
1104.8 \\
349.4 \\
646.8\end{array}$ & $\begin{array}{r}38 \\
100 \\
39 \\
20 \\
10\end{array}$ & $\begin{array}{r}29^{\circ} \\
75^{\circ} \\
30^{\circ} \\
15^{\circ} \\
8^{\circ}\end{array}$ & $\begin{array}{r}6.9 \\
11.8 \\
36.8 \\
23.3 \\
80.9\end{array}$ & $\begin{array}{r}264 \\
1183 \\
1436 \\
466 \\
809\end{array}$ \\
\hline $\begin{array}{l}37 \\
22 \\
45 \\
44 \\
43\end{array}$ & $\begin{array}{r}30.0 \\
30.0 \\
150.0 \\
100.0 \\
150.0\end{array}$ & $\begin{array}{r}26.0 \\
7.0 \\
26.0 \\
32.0 \\
18.0\end{array}$ & $\begin{array}{l}0.3 \\
0.3 \\
0.5 \\
0.5 \\
0.6\end{array}$ & $\begin{array}{r}175.4 \\
749.3 \\
942.5 \\
478.1 \\
1433.6\end{array}$ & $\begin{array}{r}12 \\
45 \\
>73 \\
92 \\
87\end{array}$ & $\begin{array}{l}9^{\circ} \\
34^{\circ} \\
55^{\circ} \\
69^{\circ} \\
87\end{array}$ & $\begin{array}{r}19.5 \\
22.0 \\
17.1 \\
6.9 \\
16.5\end{array}$ & $\begin{array}{r}234 \\
992 \\
1250 \\
638 \\
1434\end{array}$ \\
\hline $\begin{array}{l}42 \\
46 \\
47 \\
17 \\
18\end{array}$ & $\begin{array}{r}200.0 \\
40.0 \\
40.0 \\
400.0 \\
250.0\end{array}$ & $\begin{array}{l}36.0 \\
17.0 \\
20.0 \\
30.4 \\
26.0\end{array}$ & $\begin{array}{l}0.5 \\
1.0 \\
0.5 \\
0.5 \\
1.0\end{array}$ & $\begin{array}{r}937.2 \\
389.8 \\
302.1 \\
2331.6 \\
1635.7\end{array}$ & $\begin{array}{r}102 \\
45 \\
52 \\
95 \\
72\end{array}$ & $\begin{array}{l}41 \\
18 \\
52 \\
60 \\
50\end{array}$ & $\begin{array}{r}22.9 \\
21.7 \\
5.8 \\
38.9 \\
32.7\end{array}$ & $\begin{array}{r}2330 \\
975 \\
302 \\
3690 \\
2360\end{array}$ \\
\hline $\begin{array}{r}66 \\
39 \\
54 \\
123 \\
121\end{array}$ & $\begin{array}{r}34.0 \\
138.0 \\
40.0 \\
22.0 \\
133.0\end{array}$ & $\begin{array}{l}72.8 \\
88.0 \\
24.0 \\
82.1 \\
31.0\end{array}$ & $\begin{array}{l}0.3 \\
0.3 \\
0.3 \\
0.3 \\
0.3\end{array}$ & $\begin{array}{r}63.8 \\
209.4 \\
235.0 \\
37.2 \\
649.0\end{array}$ & $\begin{array}{r}35 \\
19 \\
17 \\
120 \\
74\end{array}$ & $\begin{array}{l}34 \\
19 \\
20 \\
20 \\
69\end{array}$ & $\begin{array}{r}1.9 \\
11.0 \\
11.8 \\
1.9 \\
9.4\end{array}$ & $\begin{array}{r}66 \\
209 \\
200 \\
223 \\
696\end{array}$ \\
\hline $\begin{array}{l}40 \\
50 \\
51 \\
53 \\
67\end{array}$ & $\begin{array}{r}41.0 \\
400.0 \\
350.0 \\
420.0 \\
33.0\end{array}$ & $\begin{array}{l}26.0 \\
30.4 \\
32.0 \\
23.0 \\
77.2\end{array}$ & $\begin{array}{l}0.3 \\
0.5 \\
0.5 \\
0.5 \\
0.8\end{array}$ & $\begin{array}{r}220.0 \\
2200.0 \\
1800.0 \\
3100.0 \\
53.9\end{array}$ & $\begin{array}{r}53 \\
95 \\
109 \\
100 \\
68\end{array}$ & $\begin{array}{l}40^{*} \\
60 \\
72 \\
65 \\
53\end{array}$ & $\begin{array}{r}5.5 \\
36.7 \\
25.0 \\
47.7 \\
1.0\end{array}$ & $\begin{array}{r}292 \\
3480 \\
2730 \\
4770 \\
69\end{array}$ \\
\hline $\begin{array}{l}59 \\
61 \\
57 \\
58\end{array}$ & $\begin{array}{l}140.0 \\
327.0 \\
212.0 \\
300.0\end{array}$ & $\begin{array}{l}12.0 \\
23.7 \\
20.0 \\
27.0\end{array}$ & $\begin{array}{l}0.3 \\
0.5 \\
1.0 \\
0.2\end{array}$ & $\begin{array}{l}1963.3 \\
2437.2 \\
1962.3 \\
1774.6\end{array}$ & $\begin{array}{r}90 \\
76 \\
103 \\
81\end{array}$ & $\begin{array}{l}73 \\
57 \\
77 \\
61\end{array}$ & $\begin{array}{l}26.9 \\
42.8 \\
25.5 \\
29.1\end{array}$ & $\begin{array}{l}2420 \\
3250 \\
2630 \\
2360\end{array}$ \\
\hline
\end{tabular}

* Assumed to be 75 percent of saturated thickness.

Table 4. --Transmissivity estimates from slug-test data in the Caguas-Juncos Valley [ft, feet; $\mathrm{ft}^{2} / \mathrm{d}$, feet squared per day]

\begin{tabular}{cccc}
\hline $\begin{array}{c}\text { Well } \\
\text { number }\end{array}$ & $\begin{array}{c}\text { Hydraulic } \\
\text { conductivity } \\
\text { calculated } \\
\text { from slug-tests } \\
\text { (ft/d) }\end{array}$ & $\begin{array}{c}\text { Aquifer } \\
\text { saturated } \\
\text { thickness } \\
(\mathrm{ft})\end{array}$ & $\begin{array}{c}\text { Apparent } \\
\text { transmissivity } \\
\left(\mathrm{ft}^{2} / \mathrm{d}\right)\end{array}$ \\
\hline 81 & 5.8 & 31.2 & \\
85 & 17.9 & 126.9 & 181 \\
88 & 4.9 & 60.8 & 2270 \\
91 & 1.1 & 96.7 & 106 \\
\hline
\end{tabular}




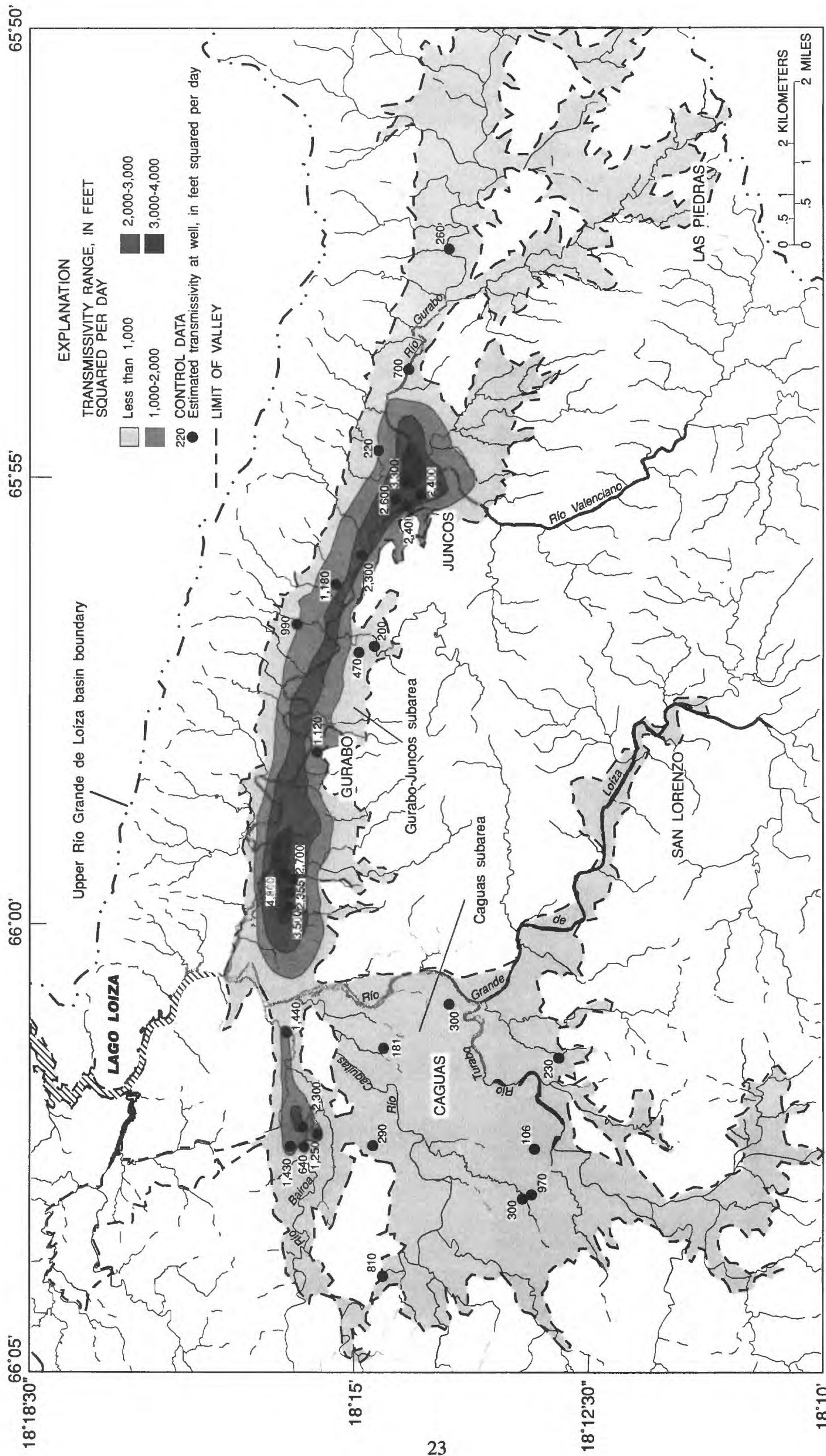

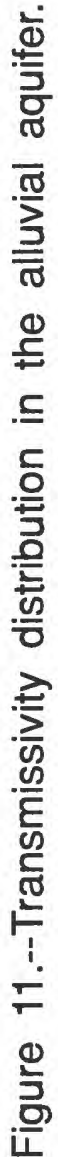


However, there are zones in Caguas, enclosing wells $46,47,48,49,90$, and 91 (fig. 7, 9, and 11), where the aquifer is thick (100 to $170 \mathrm{ft}$ ) and the transmissivity is low (less than $500 \mathrm{ft}^{2} / \mathrm{d}$ ).

The specific yield (Sy) of a formation is the ratio of the volume of water that, after saturation, can be drained by gravity to its own volume (Todd, 1980, p. 37). Although values for $\mathrm{Sy}$ of the aquifer have not been derived from aquifer tests in the study area, estimates can be obtained on the basis of porosity and specific retention inferred from the lithology. For instance, clay materials have low Sy values of about 0.03 , while medium sand has higher Sy values of about 0.28 (Todd, 1980). The aquifer in the Caguas subarea has a high clay content, but in the Gurabo-Juncos subarea, the aquifer contains a higher percentage of sand. Based on available lithologic data, the Sy for the aquifer was inferred to be about 0.10 in the Caguas subarea and about 0.15 in the Gurabo-Juncos subarea. Other authors have reported Sy values of the same order for comparable alluvial aquifers in the island. For instance, Díaz and Jordan (1987) reported 20 percent $(0.20)$ for the lower the Río Grande de Añasco Valley, Quiñones-Aponte (1986) mentioned a Sy of 0.15 for the Río Yauco alluvial valley, and Crooks and others (1968) stated a value of 0.05 for the Guayanilla-Yauco area.

\section{Water Occurrence and Movement}

In the Caguas-Juncos Valley, ground water occurs mostly under unconfined conditions, but semi-confined conditions do occur, especially away from the principal stream channels in the Caguas subarea. However, the aquifer is in hydraulic connection with most surface water bodies.

A potentiometric surface map constructed using ground-water levels measured in wells and piezometers reveals the generalized direction of ground-water flow at the time of the measurements. Potentiometric maps showing the elevation of the water table or pressure surface in the study area during July 27-29, 1987 and March 24-27, 1988 are shown on figures 12 and 13. The ground-water system was assumed to be in a steady-state condition during these dates, because streamflow was approaching base-flow conditions.

Regional ground-water movement in the Caguas subarea differs from that of the Gurabo-Juncos subarea. In the Caguas subarea, ground water moves mostly from southwest to northeast in the southern part of the valley and from west to east in the northern part (figs.
12 and 13). Ground water also moves parallel to the Río Grande de Loíza, along the eastern edge of the Caguas subarea. In the Gurabo-Juncos subarea, the regional ground-water movement is along the main channel of the Río Gurabo, but it is also characterized by relatively shorter flow paths moving from the northern and southern boundaries of the aquifer toward the Río Gurabo (figs. 12 and 13).

The hydraulic gradient in the alluvial aquifer in the Caguas subarea ranged from 16 to $96 \mathrm{ft} / \mathrm{mi}$ and averaged about $49 \mathrm{ft} / \mathrm{mi}$, as interpreted from both potentiometric surfaces of the area (figs. 12 and 13). In the Gurabo-Juncos subarea, the hydraulic gradient is less, averaging about $28 \mathrm{ft} / \mathrm{mi}$. Figure 14 shows a comparison of the potentiometric surface along a regional flow line in each valley, estimated from the potentiometric surface of March 24-27, 1988.

Average interstitial velocity may be approximated from aquifer porosity, the hydraulic gradient shown on the potentiometric map of March 24-27, 1988 (fig. 13), and the hydraulic conductivity determined from specific capacity and slug tests (tables 3 and 4). Aquifer porosity for the Caguas and Gurabo-Juncos subareas, was estimated from lithologic information and values of porosity for unconsolidated deposits reported by Freeze and Cherry (1979). Velocities were then estimated at selected sites within the aquifer using the following equation:

$$
v=k / n(\Delta h / \Delta l),
$$

where

$v \quad$ is the average interstitial velocity $(\mathrm{ft} / \mathrm{d})$,

$k \quad$ is the hydraulic conductivity ( $\mathrm{ft} / \mathrm{d}$ ),

$n \quad$ is the porosity, and

$\Delta h / \Delta l$ is the hydraulic gradient ( $\mathrm{ft} / \mathrm{ft})$.

Calculated velocities ranged between 0.04 to $1.48 \mathrm{ft} / \mathrm{d}$ (table 5).

The potentiometric surface of an aquifer is continuously changing owing to variable recharge and discharge. The wells and piezometers were monitored, either continuously or on a periodic basis, in order to observe changes in the water table. Representative hydrographs of selected wells are shown in figures 15 through 21. Low water levels for the year usually occurred in the spring and fall, and normally correspond to the end of a period of little or no rainfall. Highest ground-water levels usually occurred during the winter, at the end of the wet season. 


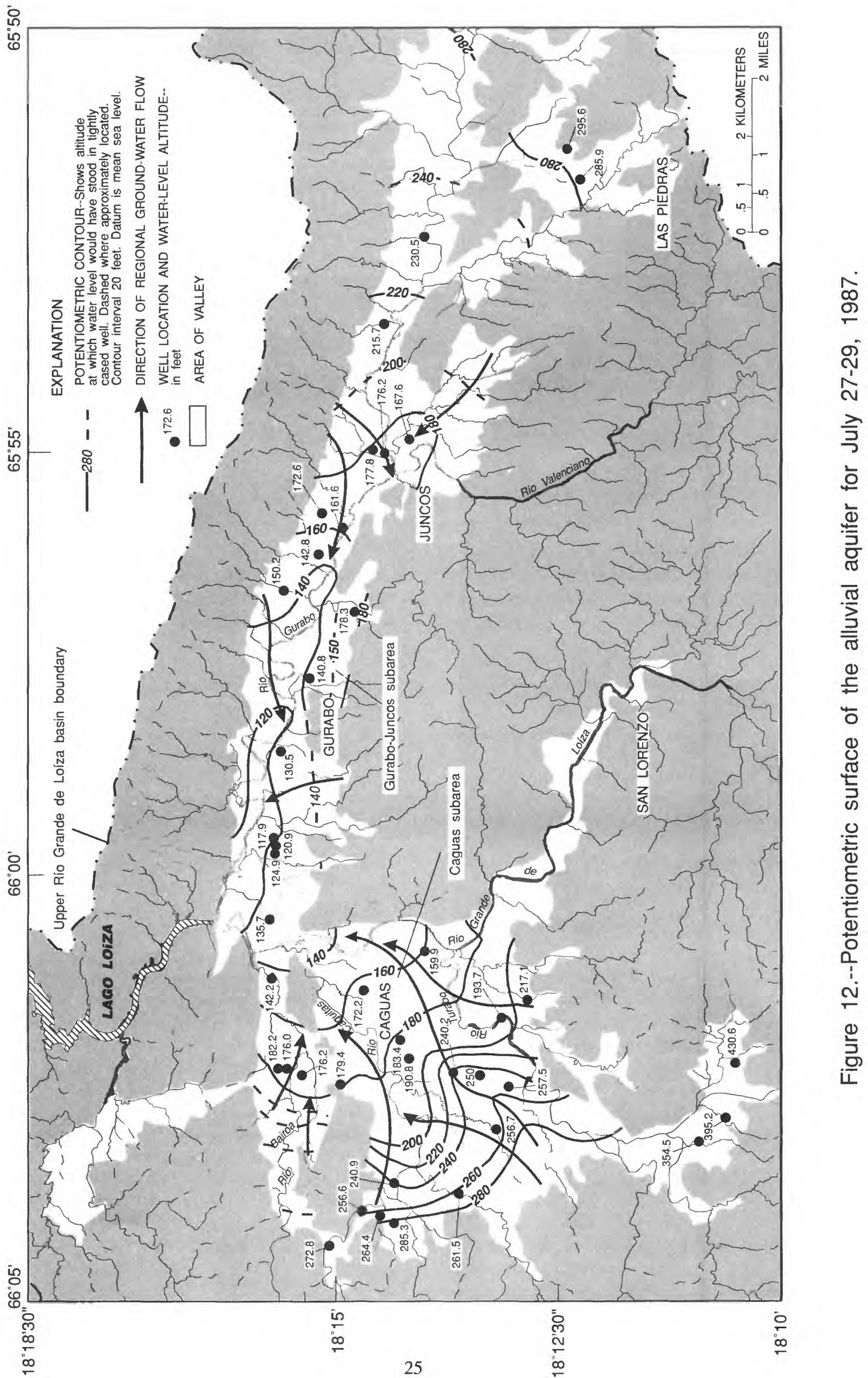


Table 5.--Estimated average interstitial velocities at selected locations throughout the Caguas-Juncos aquifer [ft/d, feet per day; ft/ft, feet per foot; \%, percent]

\begin{tabular}{|c|c|c|c|c|}
\hline $\begin{array}{l}\text { Number of } \\
\text { nearby well }\end{array}$ & $\begin{array}{l}\text { Hydraulic } \\
\text { conductivity } \\
(\mathrm{ft} / \mathrm{d})\end{array}$ & $\begin{array}{l}\text { Hydraulic } \\
\text { gradient } \\
\text { (ft/ft) }\end{array}$ & $\begin{array}{l}\text { Assumed } \\
\text { porosity } \\
\text { range (\%) }\end{array}$ & $\begin{array}{c}\text { Velocity } \\
\text { range } \\
(\mathrm{ft} / \mathrm{d})\end{array}$ \\
\hline $\begin{array}{ll}42, & 44 \\
40 & \\
46, & 47 \\
88 & \\
17, & 18\end{array}$ & $\begin{array}{r}14.9 \\
5.5 \\
13.8 \\
4.9 \\
35.8\end{array}$ & $\begin{array}{r}0.0048 \\
.0040 \\
.0076 \\
.0042 \\
.0131\end{array}$ & $\begin{array}{l}30-51^{1} \\
30-51 \\
30-51 \\
30-51 \\
33-54^{2}\end{array}$ & $\begin{array}{r}0.14-0.24 \\
.04-.07 \\
.21-.35 \\
.04-.07 \\
.87-1.48\end{array}$ \\
\hline $\begin{array}{l}54 \\
85 \\
61 \\
30 \\
65\end{array}$ & $\begin{array}{r}11.8 \\
17.9 \\
42.8 \\
11.8 \\
6.9\end{array}$ & $\begin{array}{l}.0137 \\
.0103 \\
.0075 \\
.0117 \\
.0089\end{array}$ & $\begin{array}{l}33-54 \\
33-54 \\
33-54 \\
33-54 \\
33-54\end{array}$ & $\begin{array}{l}.30-.42 \\
.34-.56 \\
.59-.97 \\
.26-.42 \\
.11-.19\end{array}$ \\
\hline
\end{tabular}

Of the four continuous record ground-water stations, two were located in the Caguas subarea, and two were located in the Gurabo-Juncos subarea, one near Gurabo and one near Juncos (fig. 7). Well 90 (CJ-TW 09), located in the center of the Caguas subarea, recorded fluctuations in ground-water levels until it was discontinued in October 1987. The observed water-level change during the time the station was in service was about $7.5 \mathrm{ft}$ (fig. 15). Well 36 (Manrique 1), is located near the Río Turabo in the Caguas subarea. The water-level record for this well showed minor fluctuations within a long-term trend caused by a stage change in the stream. The net change in water level in well 36 from 1986 to 1988 was about $2.0 \mathrm{ft}$ (fig. 15).

The water-level record at the two ground-water stations located in the Gurabo-Juncos subarea (wells 84 and 17) showed fluctuations of about $10 \mathrm{ft}$ from the beginning of 1986 to the end of 1988 (fig. 16). Observation well 84 (CJ-TW 3B) is located near Juncos far from any pumping center, but in an area where rainfall infiltration recharges the aquifer. Well 17 (Gurabo-USGS) is located near the Gurabo PRASA well battery, and water levels in this well are influenced by the pumping of nearby production wells.

The direction of vertical ground-water movement at selected wells along section B-B' near Juncos is shown in figure 17. The ground-water gradient is downward near wells 83 and 84 and upward near wells 85 and 86 , due to the effect of recharge and discharge in the aquifer.
Hydrographs based on water levels measured periodically (at about 2-month intervals) during 1987 to 1988 in 20 wells are presented in figures 18 through 21. Some active wells were measured during pumping and static conditions. The minimum observed change in static water levels for the period of record was 1.5 $\mathrm{ft}$ in well 48 (Hospital Regional 2) (fig. 18); the maximum observed change was $11 \mathrm{ft}$ in well 63 (Cardona) (fig. 19); but, the average change was about $4 \mathrm{ft}$.

\section{Stream-Aquifer Relations}

If an aquifer is in hydraulic connection with a stream, the aquifer may either gain water from the stream or lose water to the stream. The amount of water exchanged is a function of the head difference between the aquifer and the stream and the hydraulic characteristics of the streambed and aquifer materials. Seepage studies, which consist of simultaneous discharge measurements made along river sections, are sometimes used to determine the net gain or loss of water along a stream reach. This net gain or loss of water represents the exchange of water between the aquifer and the stream. The seepage data have to be interpreted with caution, however, because the amount of water gained or lost along a reach may be small in comparison to the error in the discharge measurement. If the error in the discharge measurement is greater than the estimated gain or loss, then nothing can be concluded about water exchanged between the stream and the aquifer. 
A seepage study was conducted in the Caguas-Juncos Valley on March 25, 1988 (fig. 13). The discharges of principal streams were measured where they enter the alluvial valley and at sites where they join with other streams, or discharge into the Lago Loíza. The results of this seepage study are summarized in table 6 . The table includes the following: the measured discharge at the stream site (for site location refer to fig. 13); tributary contribution to the stream, which is not computed as stream gain; stream net gain or loss from or to the aquifer, which is calculated as the difference between two contiguous measurement sites minus the tributary contribution; the percent range of stream discharge gain or loss, which is calculated as the ratio of stream net gain or loss to measured discharge at the stream; the distance between sites; and the width and average depth of the measurement site.

The error associated with the discharge measurements was estimated to range from 5 to 8 percent. However, taking into consideration these errors, some stream reaches did show a significant gain or loss. A gain or loss was considered significant if it exceeded 8 percent of the stream discharge and small changes (less than 8 percent) were considered as being within the range of possible measurement error (table 6). For instance, along the Río Gurabo, two of the 10 measured reaches, the uppermost reach and the next to the most downstream reach, showed a gain greater than 8 percent. Río Valenciano, with two measured reaches, did not show a gain or loss greater than 8 percent of the stream discharge. The Río Grande de Loíza, the largest stream in the valley, had a significant gain $\left(9.0 \mathrm{ft}^{3} / \mathrm{s}\right)$ only in the uppermost reach. For the two measured reaches along the Río Turabo, the upper reach had a gain of $+1.50 \mathrm{ft}^{3} / \mathrm{s}$, and the lower reach had a loss of $-1.18 \mathrm{ft}^{3} / \mathrm{s}$. The Río Cagüitas had significant gains at the two measured reaches. Only the uppermost reach of the Río Bairoa, the smallest of the measured streams, showed a significant gain. Most gaining reaches of the streams measured were in the upper parts of the stream where the stream enters the valley. The amount of water exchanged in the lowermost reaches generally was small.

In humid Puerto Rico, some streams discharge to aquifers but out of nine measured reaches with significant gains or losses in the study area, seven showed stream gains (table 6). The largest stream gain was observed along the uppermost reach of the Río Grande de Loíza $\left(9.0 \mathrm{ft}^{3} / \mathrm{s}\right)$. The largest significant stream loss was $-2.18 \mathrm{ft}^{3} / \mathrm{s}$, observed at a middle reach of the Río Gurabo. Some streams lose water to the aquifer where the water table may be lower than the stream stage, but may regain water in a downstream reach.

When all measured stream inflows to the valley were added together and the sum of all measured stream outflows are subtracted, the resulting value provides a quantitative estimate of the net gain or loss of the streams traversing the valley. For the seepage study of March 25, 1988, a time of low-flow conditions, the net stream gain in the Caguas-Juncos Valley was about $23 \mathrm{ft}^{3} / \mathrm{s}$. Stream seepage data for high-flow conditions are not available, but it is expected that during high-flow periods, the streams will recharge the aquifer.

\section{Water Storage and Balance}

The amount of water in storage in the alluvial aquifer is a function of the volume of saturated alluvial material. The water in storage in the aquifer was estimated from the generalized thickness of the alluvium (fig. 9), the percent of saturated alluvium based on the potentiometric surface in March 1988 (fig. 13), the estimated specific yield of the aquifer, and the aquifer surface area.

In the Caguas subarea, the average thickness of saturated alluvial material is about $44 \mathrm{ft}$, the total area underlain by the alluvial aquifer is about 10,620 acres, and the specific yield of the aquifer is assumed to be 10 percent; thus, about 46,700 acre-ft of water was in storage in March 1988. Based on the same type of calculation, the aquifer in the Gurabo-Juncos subarea contained about 75,200 acre- $\mathrm{ft}$ of water. The amount of water in storage in the alluvial aquifer totaled about 122,000 acre-ft. A 5- $\mathrm{ft}$ change in the water levels of these alluvial aquifers would change the volume of ground water in storage by about 5,300 acre- $\mathrm{ft}$ in the Caguas subarea and about 8,900 acre- $\mathrm{ft}$ in Gurabo-Juncos subarea.

The components of a water budget for the Caguas-Juncos Valley are classified either as sources or sinks. The sources to the system include total rainfall over the aquifer surface area, bedrock seepage along the aquifer boundaries, and water flowing into the aquifer from the streams. The sinks include well pumpage, net seepage from the aquifer to the streams, overland runoff, and evapotranspiration. Depending on location, the streams can be both sources and sinks. 
$66^{\circ} 05^{\prime} \quad 66^{\circ} 00^{\prime}$

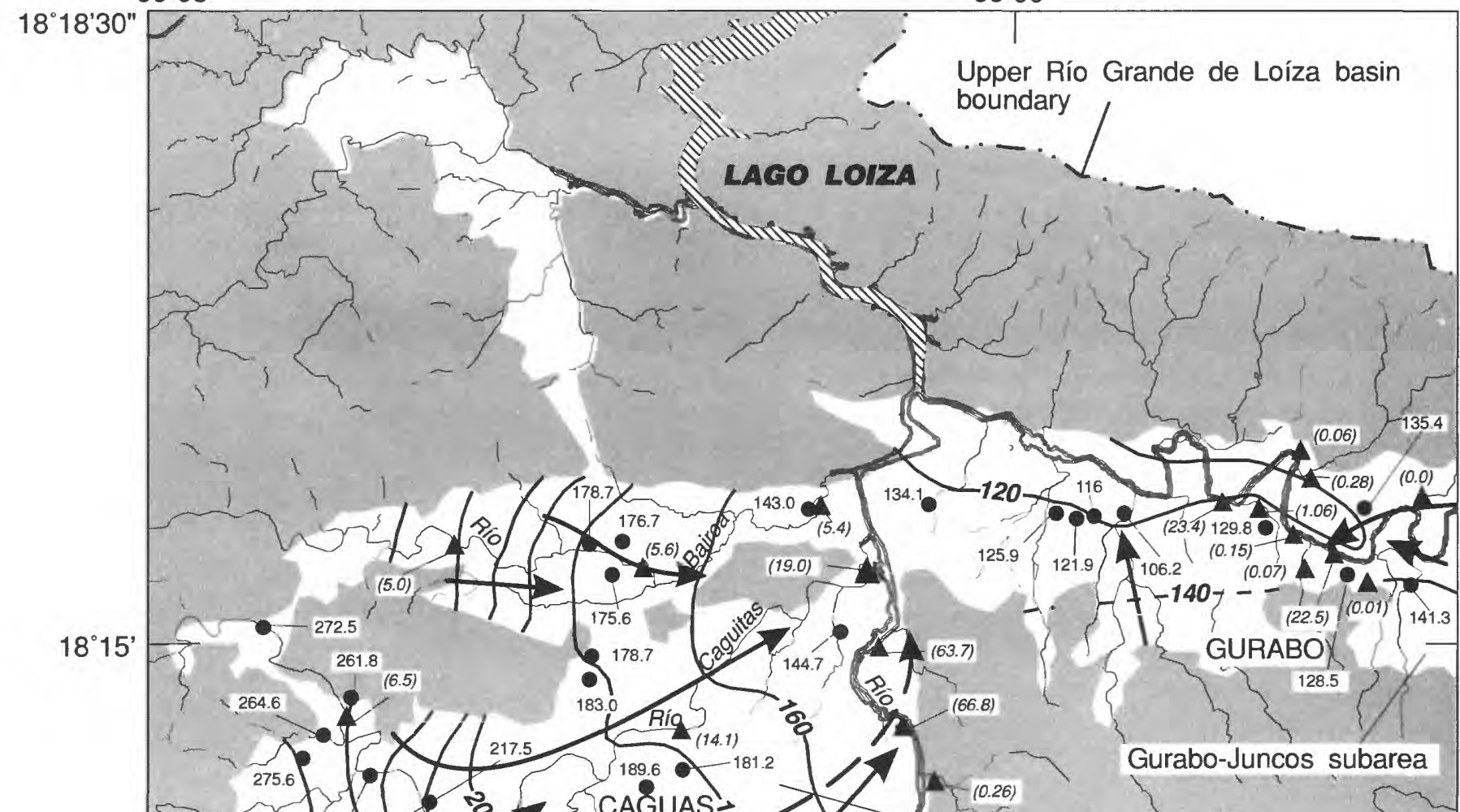

$18^{\circ} 12^{\prime} 30^{\prime \prime}$

$18^{\circ} 10^{\prime}$

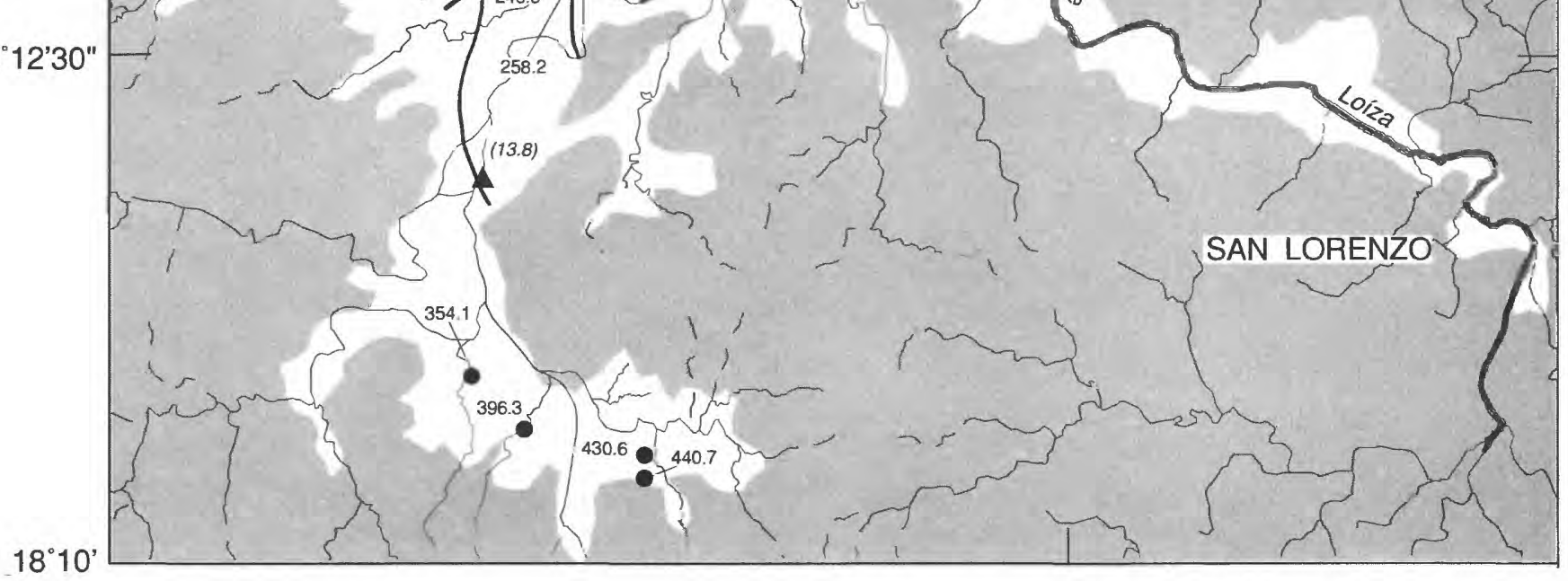

Figure 13.--Potentiometric surface and stream discharges within the alluvial aquifer for March 24-27, 1988. 


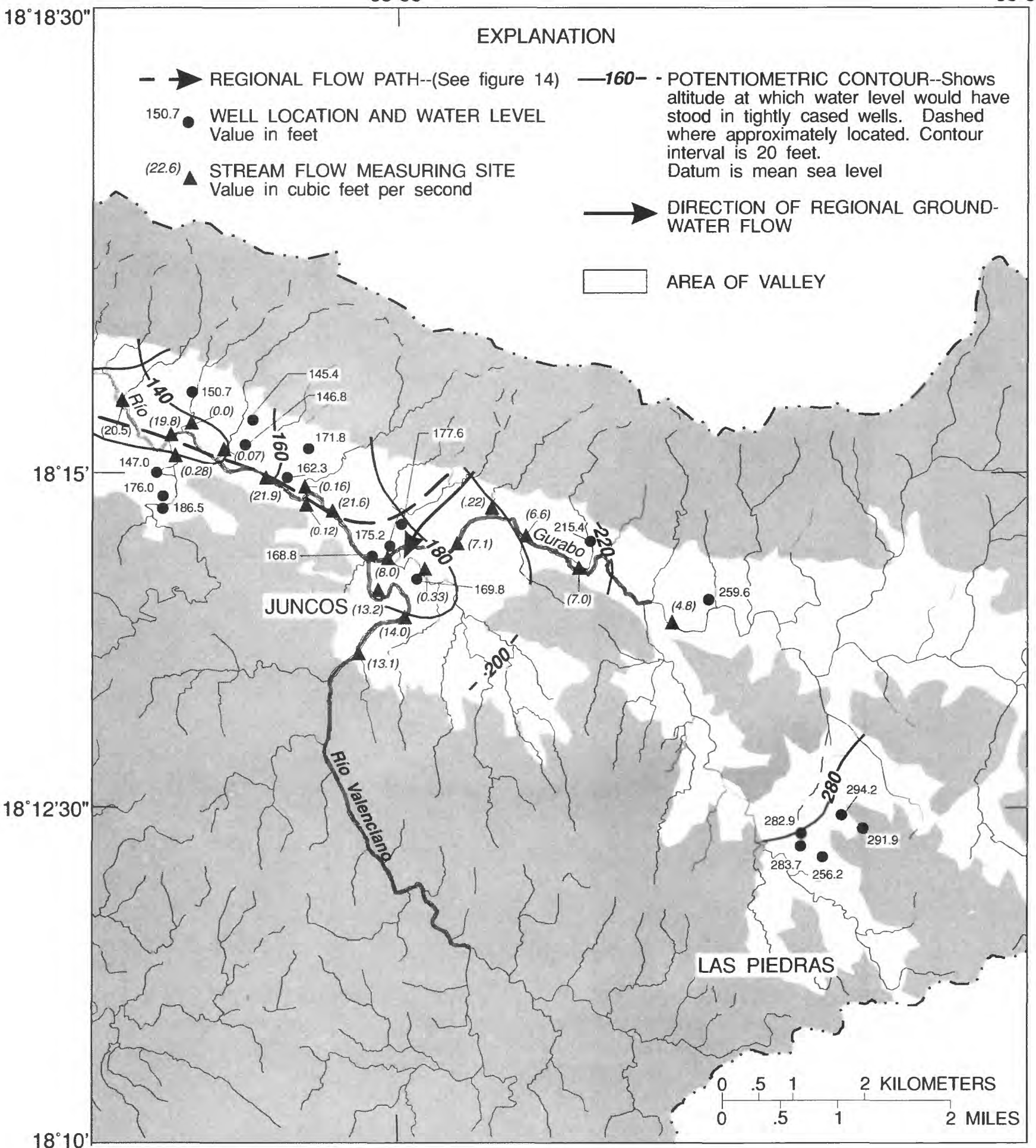

Figure 13.--Potentiometric surface and stream discharges within the alluvial aquifer for March 24-27, 1988--(Continued). 


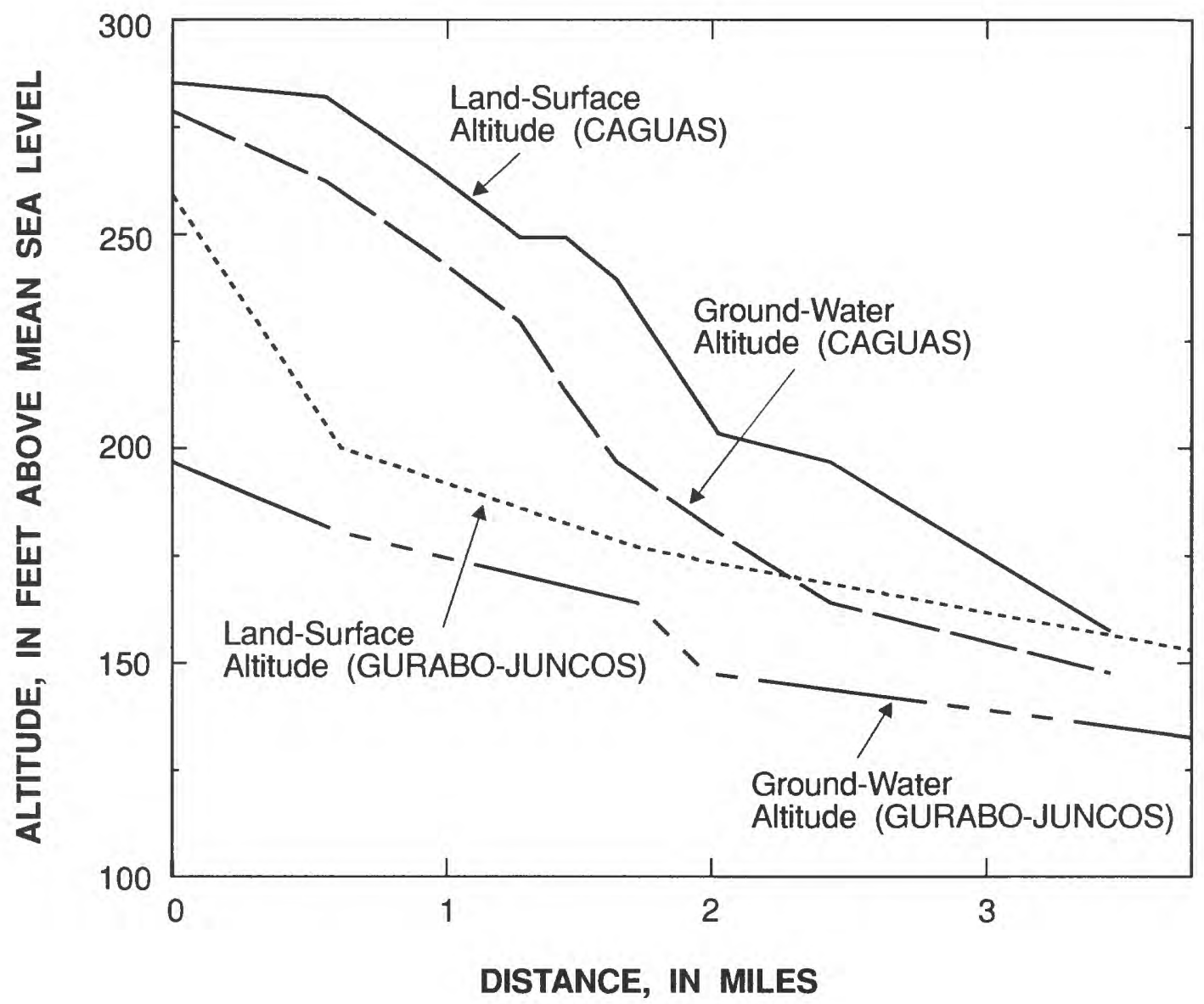

Figure 14.--Relation between land surface and the potentiometric surface along regional flow paths in the Caguas and Gurabo-Juncos subareas, March 24-27, 1988 (Location of flow paths are shown in figure 13). 


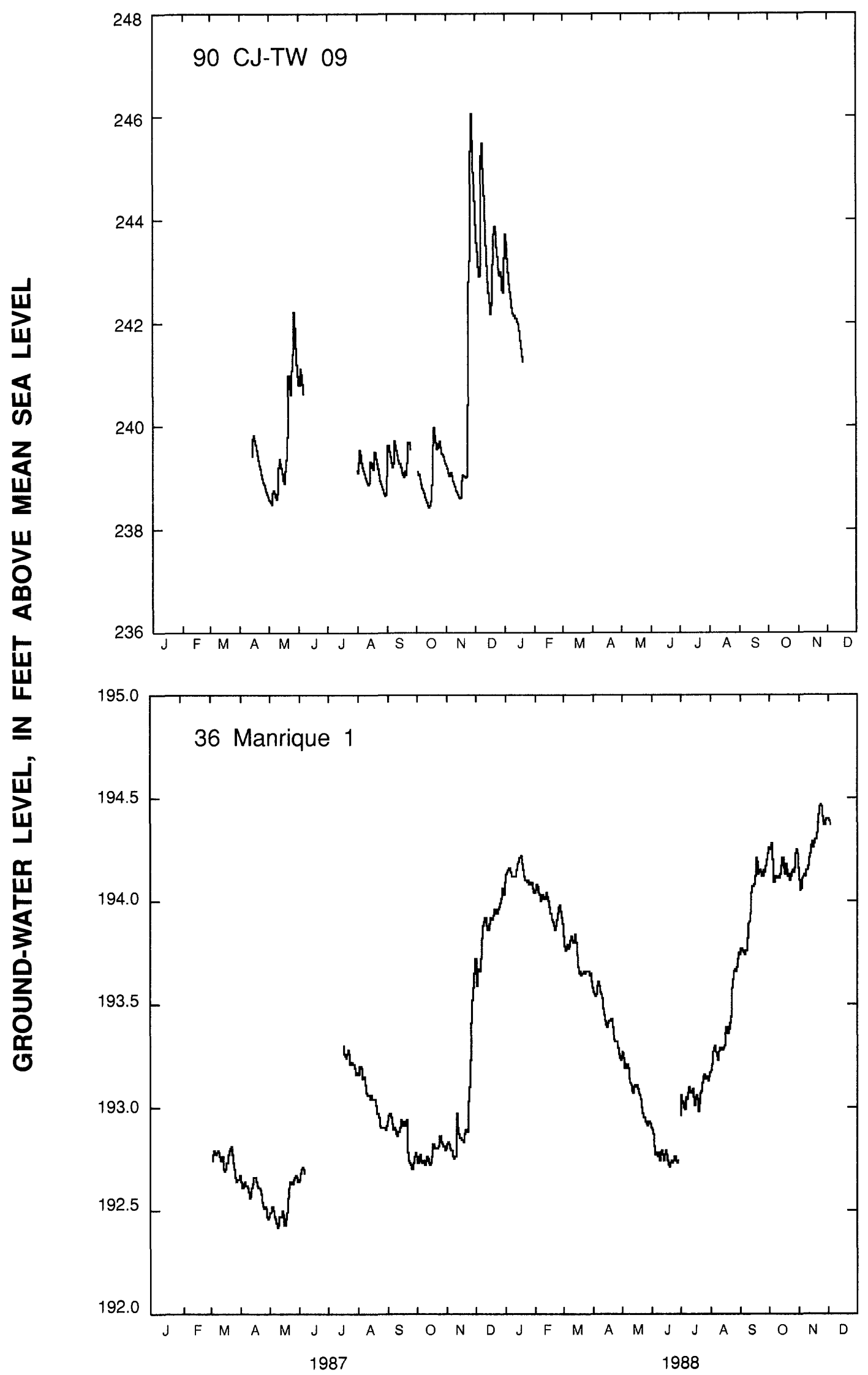

Figure 15.--Water levels in wells $90 \mathrm{CJ}-\mathrm{TW} 09$ and 36 Manrique 1, 1987-1988. 

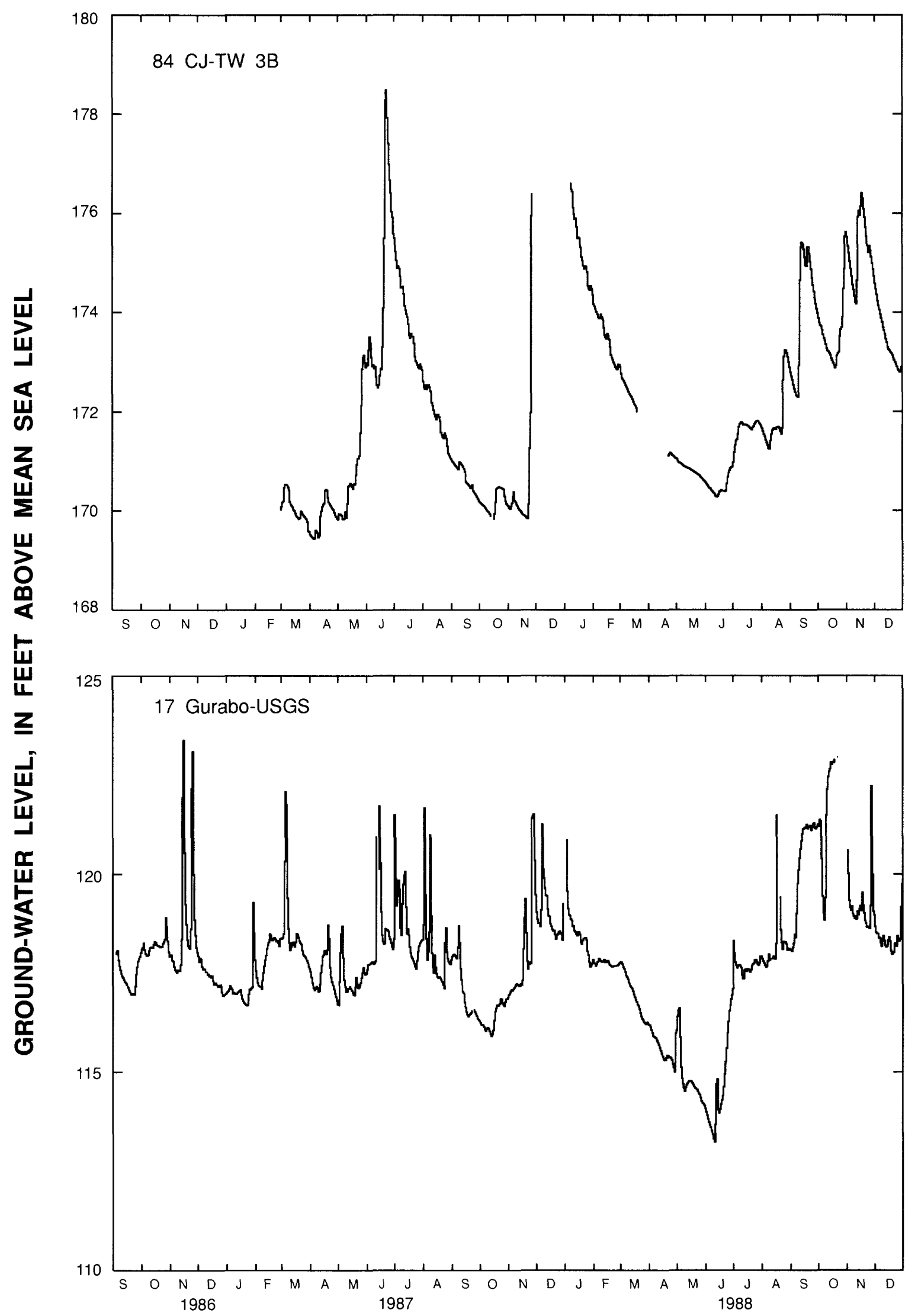

Figure 16.--Water levels in wells 84 CJ-TW 3B and 17 Gurabo-USGS, 1986-1988. 


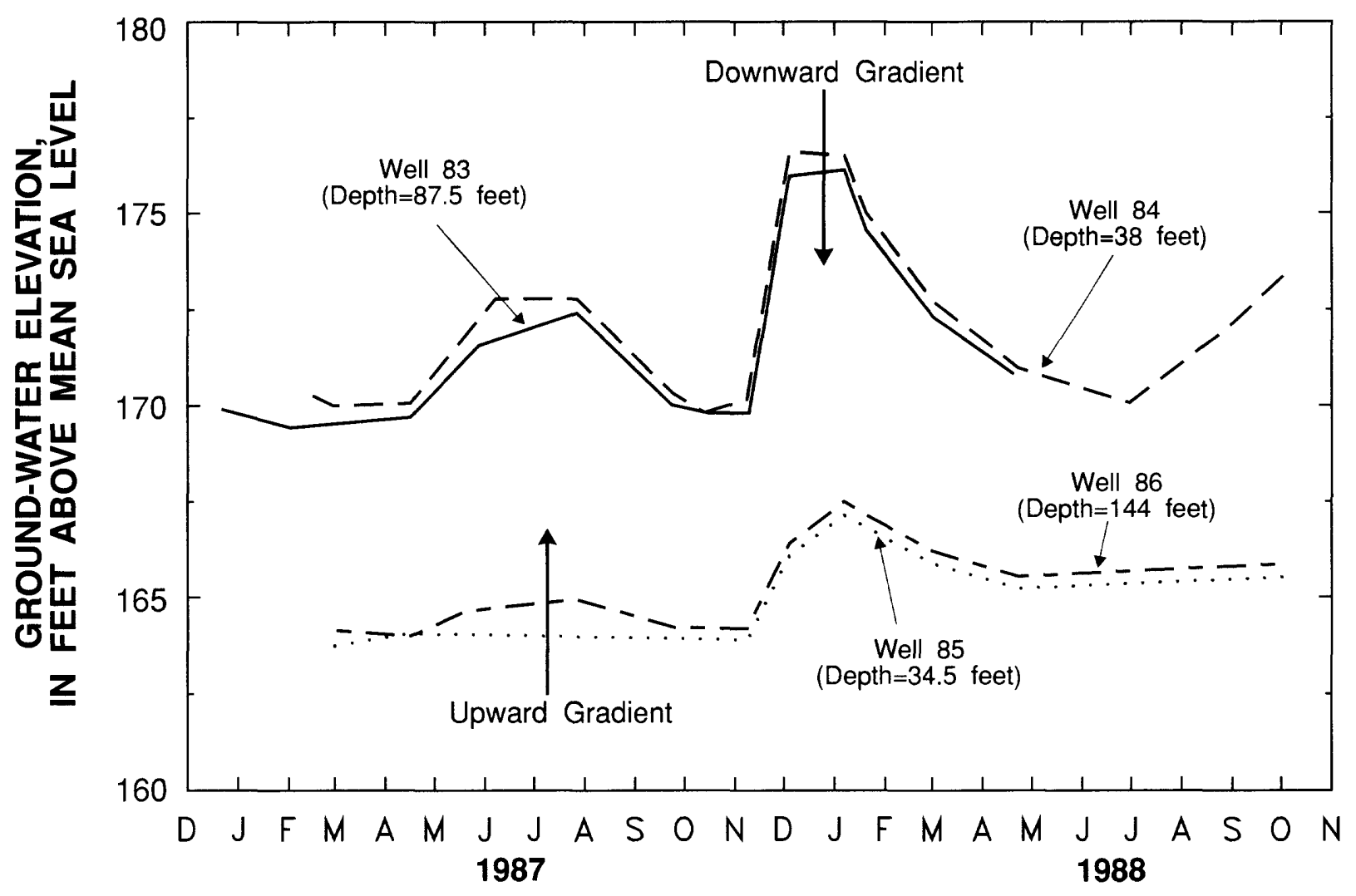

Figure 17.- Water levels in shallow and deep wells along hydrogeologic section B-B' near Juncos showing the direction of vertical movement of water (Refer to figure 7 for relative location of wells). 

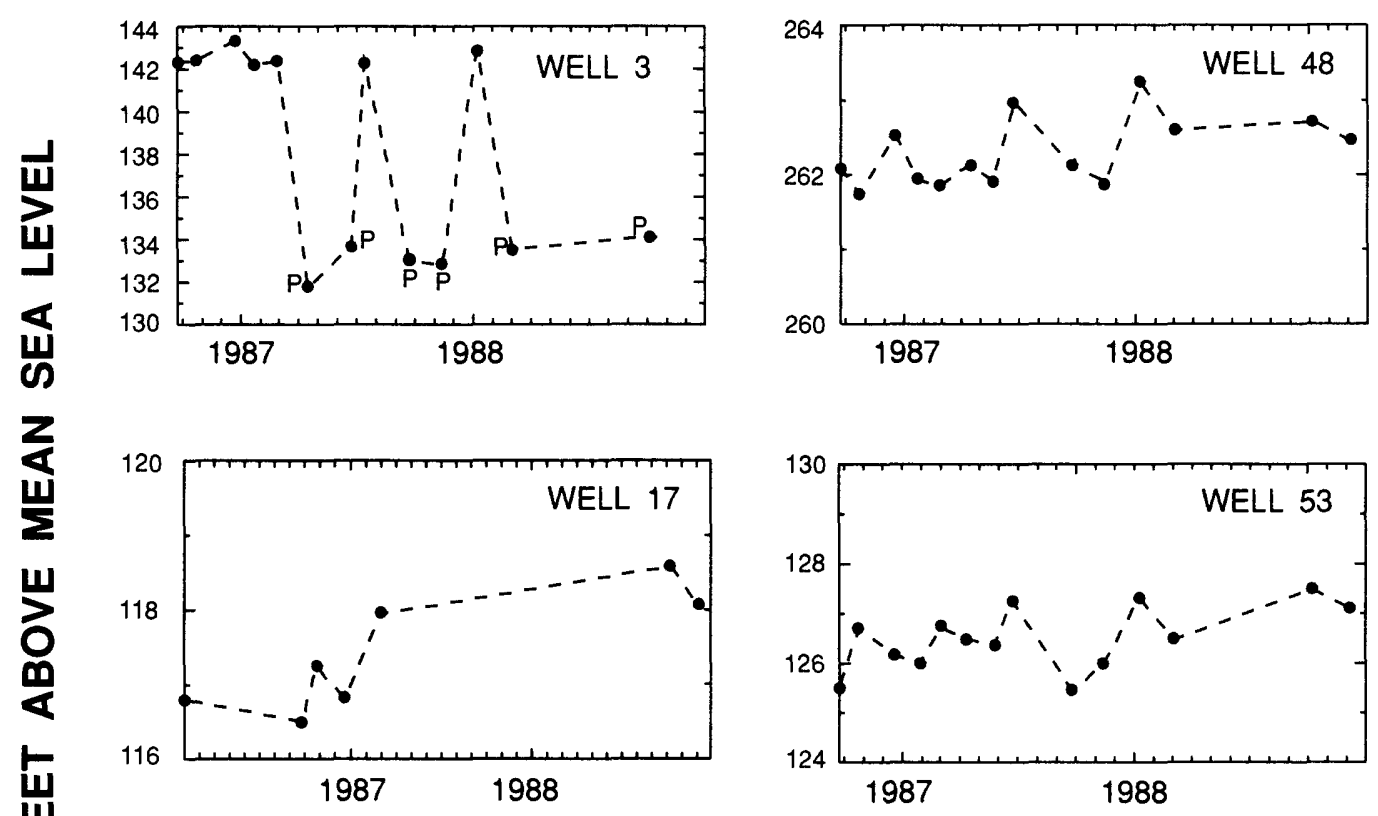

L.
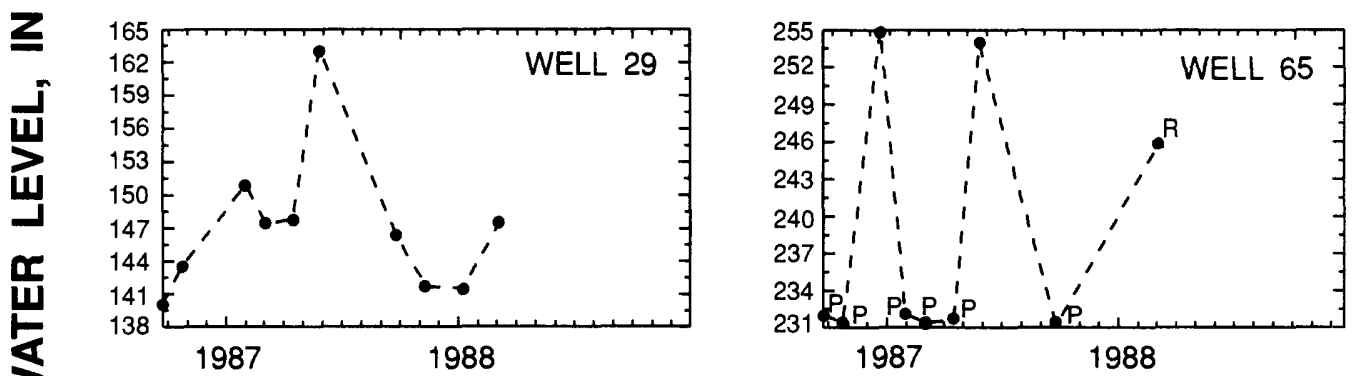

$\frac{2}{2}$
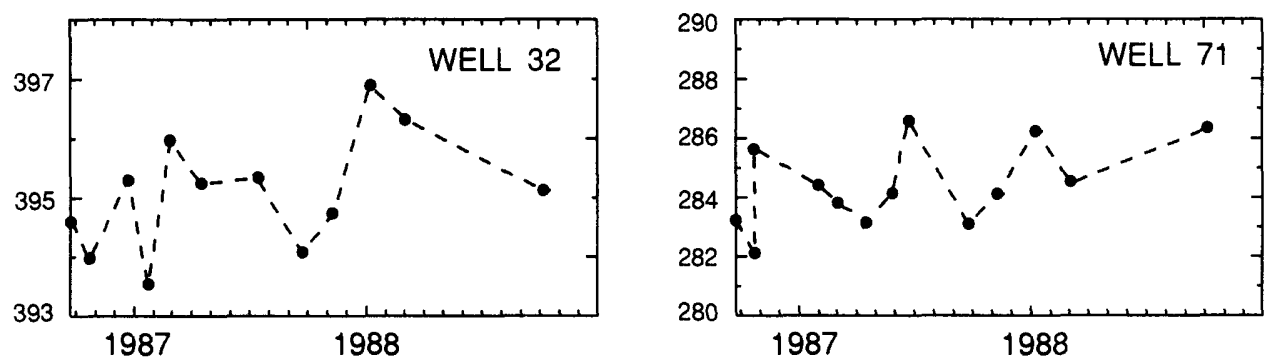

\section{EXPLANATION}

- Static water level

P. Pumping water level

R. Recovery water level

Figure 18.--Periodic ground-water levels for wells $3,17,29,32,48,53$, 65 , and 71. 

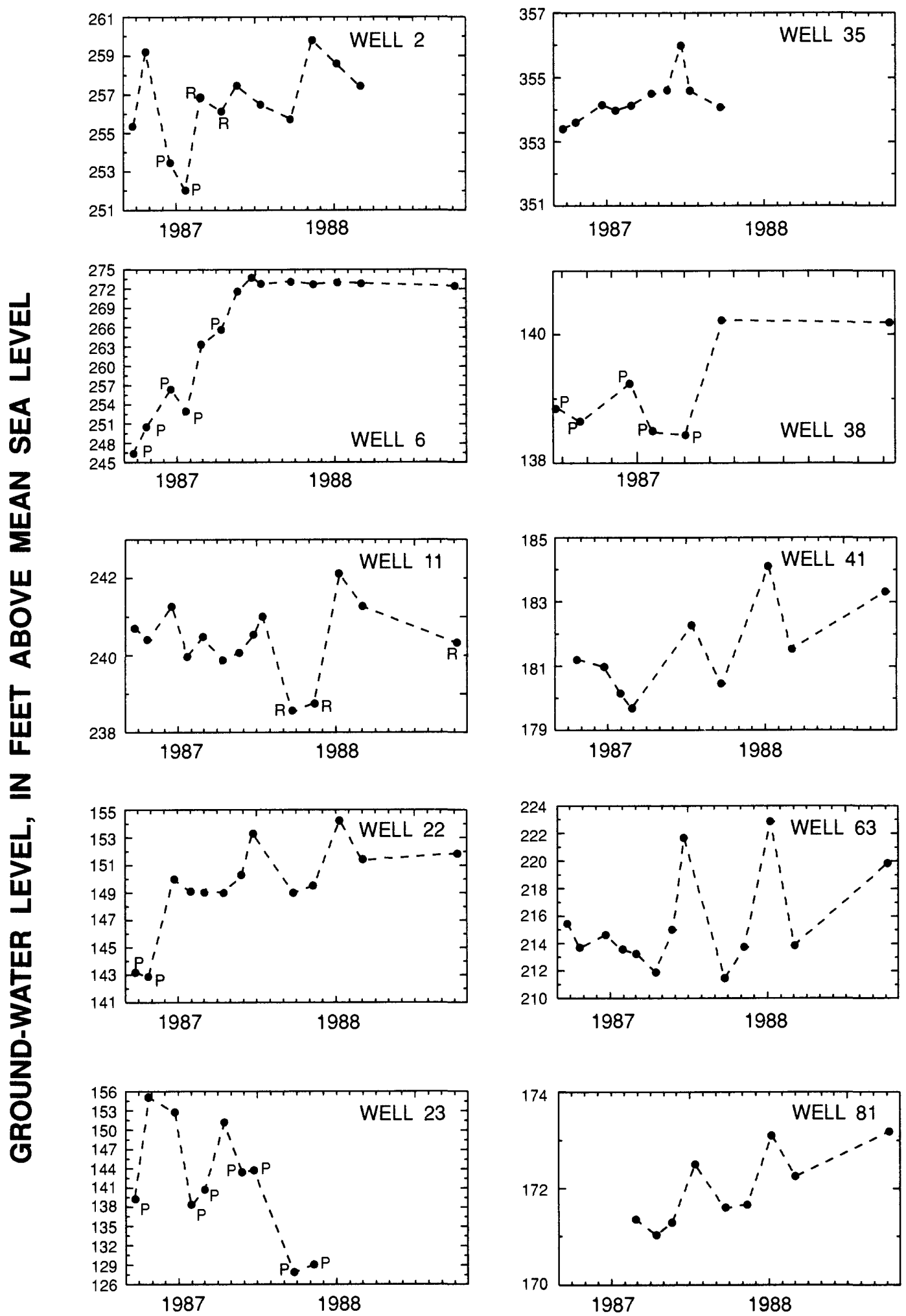

\section{EXPLANATION}

- Static water level

P. Pumping water level

R. Recovery water level

Figure 19.--Periodic ground-water levels for wells $2,6,11,22,23,35,38,41$, 63 , and 81. 

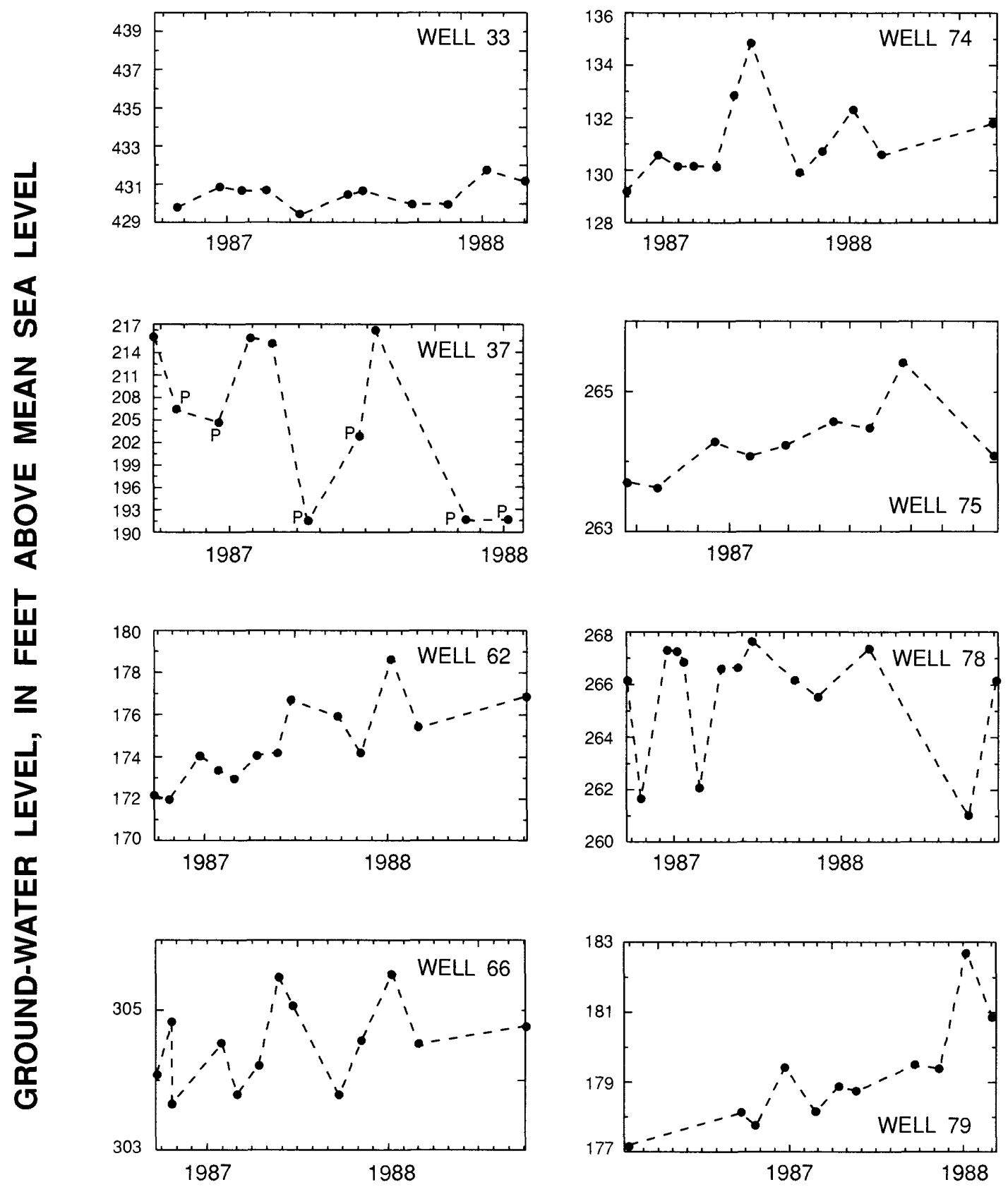

\section{EXPLANATION}

- Static water level

P. Pumping water level

Figure 20.--Periodic ground-water level for wells $33,37,62,66,74,75$, 78 , and 79. 

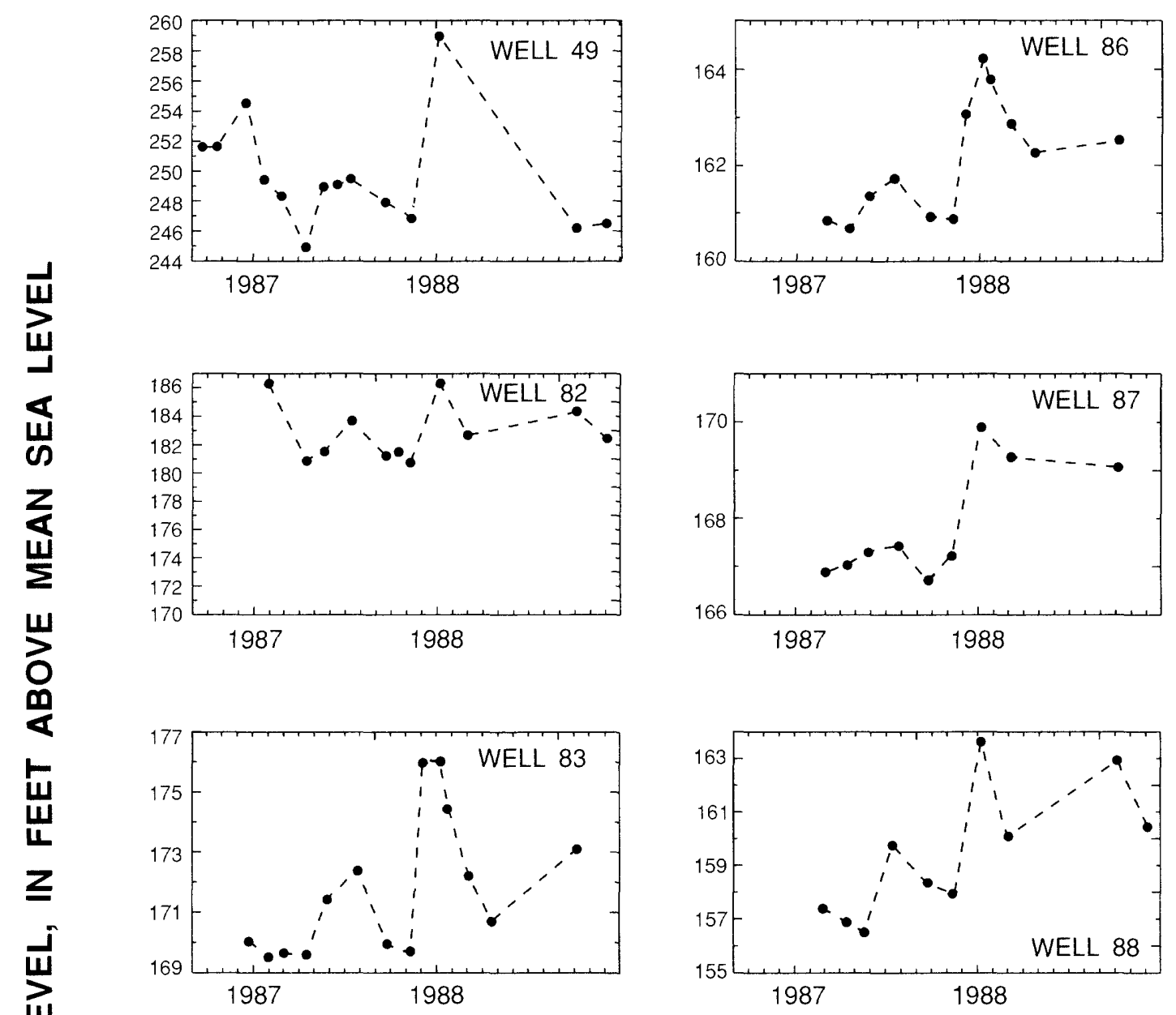

䍃
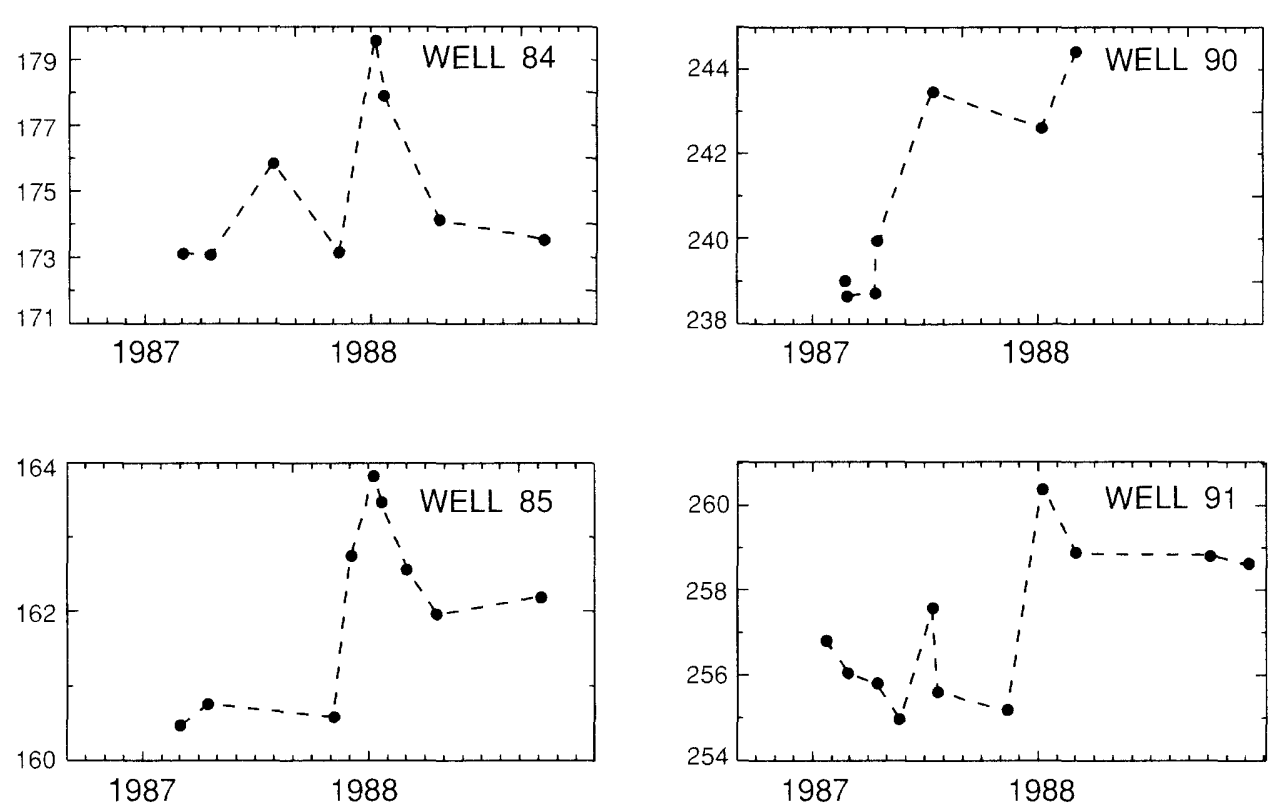

\section{EXPLANATION}

- Static water level

Figure 21.--Periodic ground-water levels for wells 49, 82, 83, 84, 85, 86, 87, 88, 90 , and 91. 
Table 6.--Results of the seepage study conducted along the principal streams flowing through the Caguas-Juncos Valley, March 25, 1988

[ $\mathrm{ft}^{3} / \mathrm{s}$, cubic feet per second]

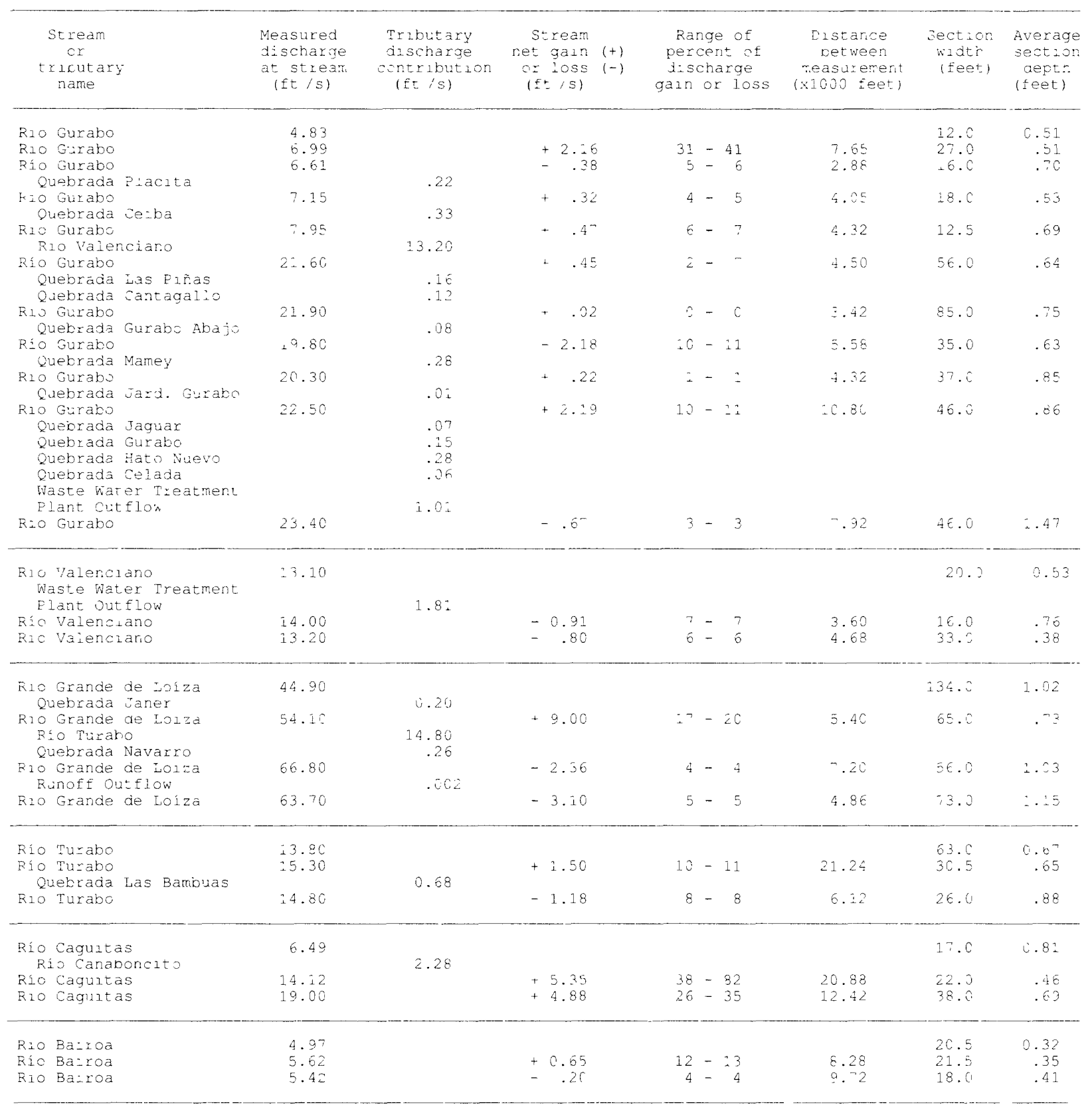


Thus, the net change in ground-water storage in the Caguas-Juncos Valley is given by the following water balance equations:

$$
\begin{gathered}
\Delta \mathrm{S}=\mathrm{BRS}+\mathrm{INF}-\mathrm{GWQ}-\mathrm{NSS}, \\
\text { and INF = RNF - ET - ROF, }
\end{gathered}
$$

where

$\triangle S$ is net change in storage,
BRS is bedrock seepage contribution,
INF is net rainfall infiltration (recharge),
GWQ is total pumpage,
NSS is net stream seepage loss,
RNF is total rainfall over the valley,
ET is evapotranspiration, and
ROF is overland runoff in the valley.

If a steady-state condition is assumed, the net change in storage is zero.

A separate water budget analysis was performed for the Caguas and the Gurabo-Juncos subareas for steady-state conditions. Total ground-water pumpage was estimated from field inspection (refer to table 2). The net steady-state stream seepage was assumed to be that measured during the seepage study conducted on March 25, 1988. Bedrock seepage contribution was estimated as 3 percent of the average precipitation over the bedrock drainage area that surrounds the alluvial aquifer (Jordan and Fisher, 1977). Overland runoff was based on average long-term streamflow at gaging stations in the valley and on seepage data of March 1988.

In the Caguas subarea, the bedrock seepage contribution (BRS) was calculated assuming an average rainfall of $100 \mathrm{in} / \mathrm{yr}$ over the surrounding drainage area of $33.8 \mathrm{mi}^{2}$, which multiplied by 3 percent gives a steady-state volume rate of 5,400 acre-ft/yr. Average rainfall (RNF) over the valley area $\left(16.6 \mathrm{mi}^{2}\right)$ was assumed to be about $60 \mathrm{in} / \mathrm{yr}$, which produced a volume rate of about 53,000 acre-ft/yr. Ground-water withdrawals (GWQ) amount to $200 \mathrm{acre}-\mathrm{ft} / \mathrm{yr}$, and the net stream seepage (NSS) from the aquifer to the stream is about $11,300 \mathrm{acre}-\mathrm{ft} / \mathrm{yr}$. An overland runoff (ROF) of about 40,500 acre-ft/yr was calculated as average stream inflows into the valley, minus outflows from the valley, minus the net stream seepage. This high rate of runoff (76 percent of rainfall) would be expected for the Caguas subarea, which is highly urbanized. If equations (4) and (5) are combined and solved for ET, and the values of all other elements of the water balance equation are substituted, the calculated ET rate in the Caguas subarea is about 6,400 acre- $\mathrm{ft} / \mathrm{yr}$ or $7 \mathrm{in} / \mathrm{yr}$. This value assumes ET only within the valley area.

In the same manner, ET was calculated for the Gurabo-Juncos subarea to be about 30,600 acre-ft/yr or $31 \mathrm{in} / \mathrm{yr}$. In the Gurabo-Juncos subarea, ET is higher than in the Caguas subarea, but runoff is lower (45 percent of rainfall). This was expected because the Gurabo-Juncos subarea has a 90 percent cover of vegetation.

Net rainfall infiltration into the aquifer can be computed by applying equation (5). Rainfall infiltration calculated by this method is about 6,100 acre- $\mathrm{ft} / \mathrm{yr}$, or 12 percent of rainfall, in the Caguas aquifer, and about 5,600 acre-ft/yr, or 8 percent of rainfall, in the Gurabo-Juncos aquifer. Evidently, ET is a more significant mechanism of water loss in the Gurabo-Juncos subarea, than in the Caguas subarea. Yet, the amount of net rainfall infiltration in both subareas is about the same: high runoff and less rainfall in the Caguas subarea compensates for the excess of ET in the Gurabo-Juncos subarea. The estimated steady-state budget for the Caguas and Gurabo-Juncos subareas, and for the overall Caguas-Juncos Valley are summarized in table 7.

The ET values ( 7 and $31 \mathrm{in} / \mathrm{yr}$ in the Caguas and Gurabo-Juncos subareas, respectively) calculated for the valley subareas were lower than the ET values (34$48 \mathrm{in} / \mathrm{yr}$ ) estimated for the whole basin, including the area outside the valley, for which the pan evaporation and the streamflow methods were used (see "Rainfall and Evapotranspiration" subsection). ET values presented in this section were based on a steady-state water balance equation and applied only to the valley area. The other ET values, based on more indirect methods, correspond to a more general or macroscopic analysis of the whole drainage basin area and do not consider land-use effects.

An independent value of rainfall infiltration into the aquifer could be obtained as a percent of total rainfall over the aquifer area, 8 percent for pasture land and 2 percent for urban area, based on studies at other aquifers in the Island (Bennett, 1976; QuiñonesAponte, 1986). In the Caguas subarea, for an average rainfall of about $60 \mathrm{in} / \mathrm{yr}$ and assuming that 60 percent of the area is covered by vegetation and that 40 percent is urban area, infiltration into the aquifer is about 3,000 acre-ft/yr. For the Gurabo-Juncos subarea, assuming that 90 percent is pasture land and 10 percent is urban area, infiltration is calculated to be about 
Table 7.--Estimated steady-state water budgets for the Caguas and the Gurabo-Juncos subareas [values are in acre-feet per year]

\begin{tabular}{|c|c|c|c|}
\hline & $\begin{array}{l}\text { Caguas } \\
\text { subarea }\end{array}$ & $\begin{array}{c}\text { Gurabo-Juncos } \\
\text { subarea }\end{array}$ & $\begin{array}{c}\text { Caguas-Juncos } \\
\text { Valley }\end{array}$ \\
\hline \multicolumn{4}{|l|}{ INELOWS: } \\
\hline $\begin{array}{l}\text { Bebrock seepage (BRS) } \\
\text { Rainfall (RNF) }\end{array}$ & $\begin{array}{l}+5,400 \\
+53,000\end{array}$ & $\begin{array}{l}+5,000 \\
+69,000\end{array}$ & $\begin{array}{l}+10,400 \\
+122,000\end{array}$ \\
\hline \multicolumn{4}{|l|}{ OUTFLOWS : } \\
\hline $\begin{array}{l}\text { Pumpage (GWQ) } \\
\text { Stream seepage (NSS) } \\
\text { Evapotranspiration (ET) } \\
\text { Runoff (ROF) }\end{array}$ & $\begin{array}{l}-\quad 200 \\
-11,300 \\
-6,400 \\
-40,500\end{array}$ & $\begin{array}{l}-3,100 \\
-7,500 \\
-30,600 \\
-32,800\end{array}$ & $\begin{array}{l}-\quad 3,300 \\
-\quad 18,800 \\
-\quad 37,000 \\
-\quad 73,300\end{array}$ \\
\hline STORAGE CHANGE: & 0 & 0 & 0 \\
\hline Net infiltration $(I N F)^{1}$ & $+6,100$ & $+5,600$ & $+11,700$ \\
\hline
\end{tabular}

5,100 acre-ft/yr. This infiltration value for the Gurabo-Juncos subarea compares favorably with the one calculated previously using equation (5) for ET. However, in Caguas, infiltration based on this method is almost 50 percent less than the previous estimate of 6,100 acre-ft/yr.

Although estimates of individual water-budget components differ depending on the method used for estimation, the order of magnitude of the estimates are similar. Given the assumptions that must be made for each method and the lack of long-term data, the water-budget estimates are the best available at this time. Nevertheless, in a future investigation, a numerical ground-water flow model may provide the necessary feedback to confirm or modify this ground-water budget.

In summary, water to the aquifer in both subareas comes from two sources in about the same magnitude, that is, from bedrock seepage and rainfall infiltration. However, aquifer outflow as stream seepage is more significant in Caguas subarea than in Gurabo-Juncos subarea, because in the latter, much of the stream seepage is intercepted by pumping wells.

\section{QUALITY OF WATER}

One of the objectives of this study was to conduct a general reconnaissance of the quality of the ground water and to provide insights into possible water-quality problems in the area. In an alluvial aquifer, such as the Caguas-Juncos, ground-water problems could not be completely separated from surface-water problems, so surface-water quality will be integrated in the discussion, as necessary.

\section{Water-Quality Analyses}

Water-quality samples were collected from ground and surface waters throughout the study area, and the results of the analyses of these samples were compared with previous analytical results reported by Gómez-Gómez and Guzmán-Ríos (1982), Guzmán-Ríos and Quiñones-Márquez (1984 and 1985), and Guzmán-Ríos and others (1986). The location of the sampled wells and surface-water sites are shown in figure 7. Results of the water-quality analyses from sampled wells and streams are summarized in tables 8 , 9 , and 10 which includes results of analyses performed during this study and those performed as part of previous ground-water reconnaissance studies. The samples were analyzed for physical, chemical, and biological characteristics, including concentrations of major ions and nutrients. Samples collected from public water-supply wells also were analyzed for organic compounds. 
Table 8. --Physical and chemical characteristics of ground water at selected wells within the Caguas-Juncos Valley

$\left[\mu \mathrm{S} / \mathrm{cm}\right.$, microsiemens per centimeter; ${ }^{\circ} \mathrm{C}$, degrees Celsius; mg/L, milligrams per liter; $\mu \mathrm{g} / \mathrm{L}$, micrograms per liter; leaders (--), no data; ND, not detected]

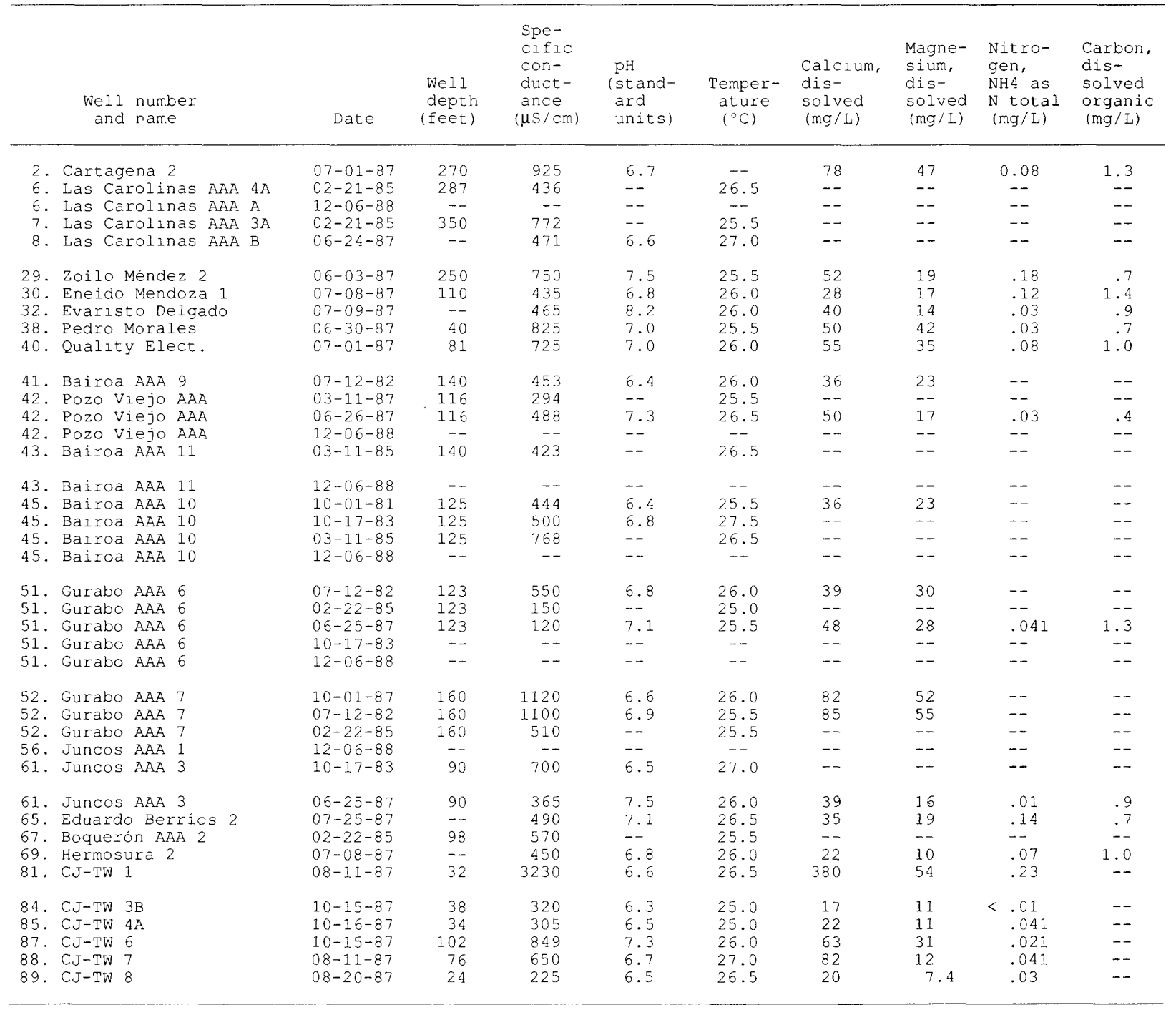


Table 8. --Physical and chemical characteristics of ground water at selected wells within the Caguas-Juncos Valley--Continued

$\left[\mu \mathrm{S} / \mathrm{cm}\right.$, microsiemens per centimeter; ${ }^{\circ} \mathrm{C}$, degrees Celsius; $\mathrm{mg} / \mathrm{L}$, milligrams per liter; $\mu \mathrm{g} / \mathrm{L}$, micrograms per liter; leaders (--), no data; ND, not detected]

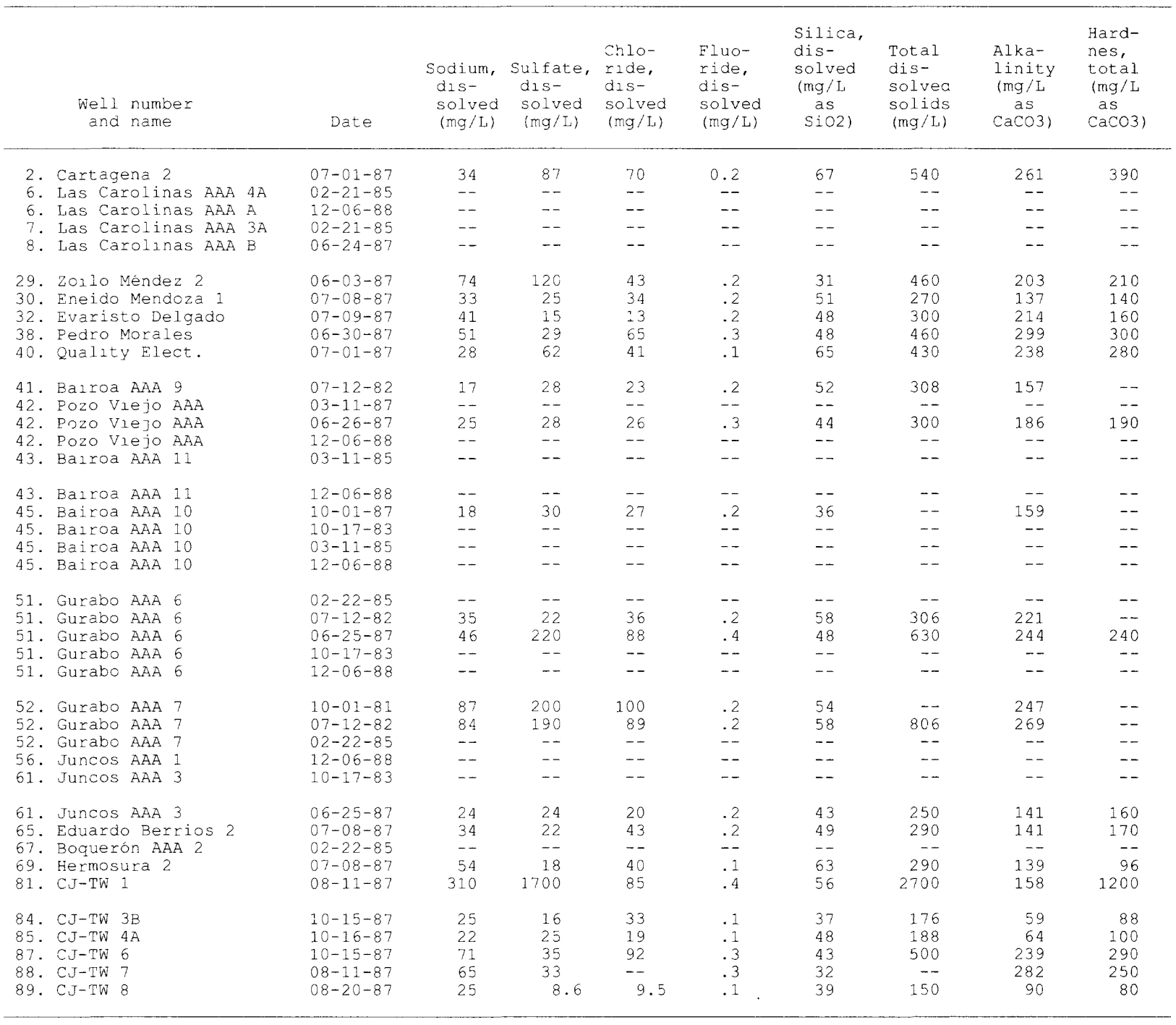


Table 8. --Physical and chemical characteristics of ground water at selected wells within the Caguas-Juncos Valley-.-Continued

$\left[\mu \mathrm{S} / \mathrm{cm}\right.$, microsiemens per centimeter; ${ }^{\circ} \mathrm{C}$, degrees Celsius; $\mathbf{m g} / \mathrm{L}$, milligrams per liter; $\mu \mathrm{g} / \mathrm{L}$, micrograms per liter; leaders (--), no data; ND, not detected]

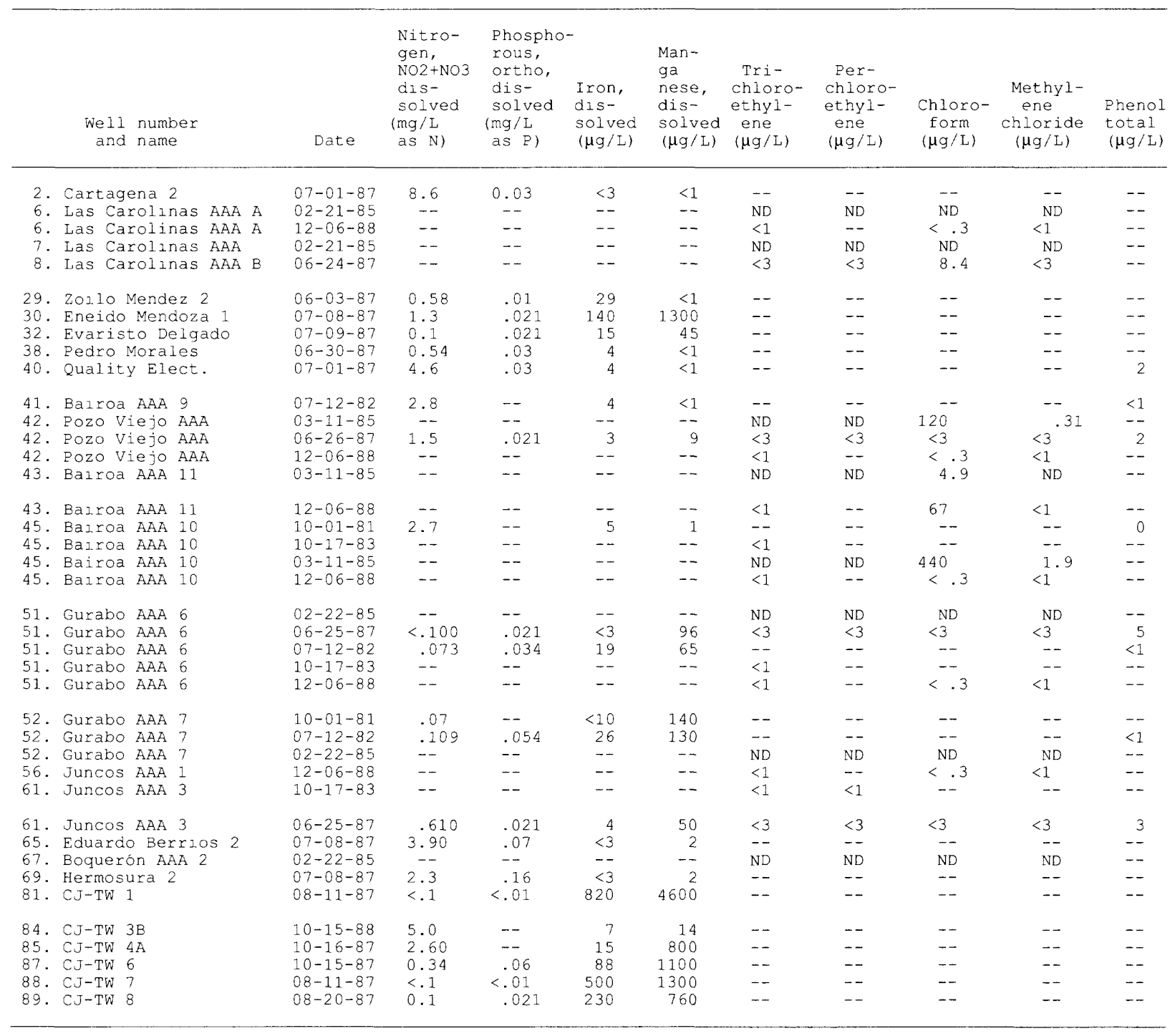

Sampling from 06-03-87 through 10-16-87 correspond to this study

Sampling from 02-21-85 through 03-11-85 by Guzmán-Ríos, René García, and Ada Aviles (1986)

Sampling on 07-12-82 by Guzmán-Ríos and Quiñones-Márquez (1984)

Sampling on 10-01-81 by Gómez-Gómez and Guzmán-Ríos (1982)

Sampling on 10-17-83 by Guzmán-Ríos and Quiñones-Márquez (1985)

Sampling on 12-06-88 by U.S. Geological Survey as part of the islandwide well monitoring program, written communication, Guzmán-Ríos (1989) 
Table 9.-- Biological characteristics of ground water at selected wells within the Caguas-Juncos Valley $[\mu \mathrm{g} / \mathrm{L}$, micrograms per liter; leaders (--), no data]

\begin{tabular}{|c|c|c|c|c|c|}
\hline \multirow{2}{*}{\multicolumn{2}{|c|}{$\begin{array}{l}\text { Well number } \\
\text { and name }\end{array}$}} & \multirow[b]{2}{*}{ Date } & \multicolumn{3}{|c|}{$\begin{array}{l}\text { Bacterial counts } \\
\text { (Colonies per } 100 \mathrm{ML} \text { of sample) }\end{array}$} \\
\hline & & & $\begin{array}{l}\text { Eecal } \\
\text { coliform }\end{array}$ & $\begin{array}{l}\text { Eecal } \\
\text { strepto- } \\
\text { cocci }\end{array}$ & $\begin{array}{l}\text { Total } \\
\text { coliform }\end{array}$ \\
\hline 41 . & Bairoa AAA 9 & $07-12-82$ & 0 & 6 & -- \\
\hline 45. & Bairoa AAA 10 & $10-01-81$ & 0 & 0 & 0 \\
\hline 51. & Gurabo AAA 6 & $07-12-82$ & 0 & 5 & -- \\
\hline 52. & Gurabo AAA 7 & $10-01-81$ & 0 & 0 & 0 \\
\hline 52. & Gurabo AAA 7 & $07-12-82$ & -- & 67 & -- \\
\hline
\end{tabular}

Sampling on 07-12-82 by Guzmán-Rios and Quiñones-Márquez (1984)

Sampling on 10-01-81 by Gómez-Gómez and Guzmán-Ríos (1982)

The data in table 8 indicate that water from several wells had high concentrations of dissolved iron and manganese. High concentrations of iron and manganese in ground water may be due to localized laterite deposits of volcanic and metamorphic origin. Water from some wells had iron or manganese concentrations that exceeded the EPA (1973) criteria of $0.3 \mathrm{mg} / \mathrm{L}$ and $0.05 \mathrm{mg} / \mathrm{L}$, respectively. These were wells $31,51,52,61,81,85,87,88$, and 89 (refer to fig. 7 for location of wells).

Specific-conductance values greater than 1,000 $\mu \mathrm{S} / \mathrm{cm}$ were detected at wells 51,52 , and 81 . No ammonia $\left(\mathrm{NH}_{4}\right)$ concentrations greater than the 0.5 $\mathrm{mg} / \mathrm{L}$ limit recommended by EPA were detected. The maximum concentration of $\mathrm{NH}_{4}(0.23 \mathrm{mg} / \mathrm{L})$ was observed in well 81 (table 8). Dissolved organic carbon concentrations ranged from 0.4 to $1.4 \mathrm{mg} / \mathrm{L}$.

Concentrations of sulfate in water from wells 29 , 51 , and 52 , were 120,220 , and $200 \mathrm{mg} / \mathrm{L}$, respectively, and the sulfate concentration in well 81 greatly exceeded the EPA criteria of $250 \mathrm{mg} / \mathrm{L}$ with a concentration of $1,700 \mathrm{mg} / \mathrm{L}$. The phosphorous concentration exceeded the EPA criteria of $0.10 \mathrm{mg} / \mathrm{L}$ only at well $69(0.16 \mathrm{mg} / \mathrm{L})$. Values for temperature, $\mathrm{pH}$, magnesium, sodium, chloride, silica, alkalinity, and nitrate+nitrite were within normal ranges in all wells monitored; none exceeded any established EPA criteria.

Public-water supply from wells were also monitored for volatile organics, including the following: trichloroethylene, perchloroethylene, chloroform, methylene chloride, and phenol.
Chloroform was present $(8.4 \mu \mathrm{g} / \mathrm{L}$ ) in well 7 (Las Carolinas AAA) in the June 1987 sampling, but was not present in samples collected between November 1984 and May 1985. Water from well 42 (Pozo Viejo AAA) contained chloroform and methylene chloride concentrations in the November 1984 to May 1985 samples, as high as 120 and $0.31 \mu \mathrm{g} / \mathrm{L}$, but the June 1987 samples contained no trace of these constituents. However, water from well 43, had a lower concentration of chloroform $(4.9 \mu \mathrm{g} / \mathrm{L})$ when sampled during the 1983 to 1984 than when sampled during $1988(67 \mu \mathrm{g} / \mathrm{L})$. The exact source of these organic compounds is unknown.

Water samples were also collected from the principal streams of the Caguas-Juncos Valley, (fig. 7 and table 10), and analyzed for physical properties, and concentrations of major ions and nutrients. The analyses indicated that four of the sampled streams (sites 2, 4, 5, and 6) had manganese concentrations ranging from 120 to $580 \mu \mathrm{g} / \mathrm{L}$ (table 10). Fecal coliform bacteria concentrations at all sites ranged from 2,200 to $520,000 \mathrm{col} . / 100 \mathrm{ml}$ (table 10), which exceeds the EPA limit of 2,000 col./100 ml for raw surface-water sources. Water samples collected during the study along the major streams of the area generally had specific-conductance values of less than 540 $\mu \mathrm{S} / \mathrm{cm}$.

Water type is commonly categorized on the basis of the composition of the principal cations and anions in the water (Piper, 1953). All but one of the ground-water samples from wells completed in the alluvium in the Caguas subarea were of the calcium 
Table 10.--Physical, chemical, and biological characteristics of water at selected streams within the Caguas-Juncos Valley $\left[\mu \mathrm{S} / \mathrm{cm}\right.$, microsiemens per centimeter; ${ }^{\circ} \mathrm{C}$, degrees Celsius; mg/L, milligrams per liter; $\mu \mathrm{g} / \mathrm{L}$, micrograms per liter]

\begin{tabular}{|c|c|c|c|c|c|c|c|c|c|c|}
\hline & $\begin{array}{l}\text { Site number } \\
\text { and name }\end{array}$ & $\begin{array}{c}\text { Station } \\
\text { Identificat }\end{array}$ & & Date & $\begin{array}{l}\text { Spe- } \\
\text { cific } \\
\text { con- } \\
\text { duct- } \\
\text { ance } \\
(\mu \mathrm{s} / \mathrm{cm})\end{array}$ & $\begin{array}{l}\mathrm{pH} \\
\text { (stand- } \\
\text { ard } \\
\text { units) }\end{array}$ & $\begin{array}{cc}\text { Pemper- } & \mathrm{C} \\
\text { ature } & \mathrm{s} \\
(\mathrm{C}) & (\mathrm{m}\end{array}$ & $\begin{array}{l}\text { Calcium, } \\
\text { dıs- } \\
\text { solved } \\
(\mathrm{mg} / \mathrm{L})\end{array}$ & $\begin{array}{l}\text { Magne- } \\
\text { sium, } \\
\text { dis- } \\
\text { solved } \\
(\text { mg/L })\end{array}$ & $\begin{array}{l}\text { Nitro- } \\
\text { gen, } \\
\text { NH4 as } \\
\text { N total } \\
(\mathrm{mg} / \mathrm{L})\end{array}$ \\
\hline \multirow[t]{2}{*}{$\begin{array}{l}\text { 1. Río } \\
\text { 2. Rio } \\
\text { 3. Rio } \\
\text { 4. Rio } \\
\text { 5. Rio } \\
\text { 6. Río }\end{array}$} & $\begin{array}{l}\text { Turabo at Caguas } \\
\text { Valenclano at mouth } \\
\text { Grande de Loiza at Caguas } \\
\text { Caguitas at Highway } 30 \\
\text { Bairoa at mouth } \\
\text { Gurabo at Gurabo }\end{array}$ & $\begin{array}{l}50054500 \\
1814110655 \\
50055000 \\
50055250 \\
50055410 \\
50057000\end{array}$ & $\begin{array}{l}07- \\
07- \\
07- \\
07- \\
07- \\
07-\end{array}$ & $\begin{array}{l}-23-87 \\
-23-87 \\
-22-87 \\
-24-87 \\
-24-87 \\
-22-87\end{array}$ & $\begin{array}{l}508 \\
291 \\
378 \\
540 \\
451 \\
302\end{array}$ & $\begin{array}{l}7.4 \\
7.7 \\
7.4 \\
7.4 \\
7.7 \\
7.5\end{array}$ & $\begin{array}{r}6.5 \\
28.5 \\
26.0 \\
30.0 \\
27.0 \\
28.5\end{array}$ & $\begin{array}{l}50 \\
19 \\
9.6 \\
38 \\
40 \\
20\end{array}$ & $\begin{array}{r}17 \\
7.1 \\
3.7 \\
13 \\
17 \\
8.9\end{array}$ & $\begin{array}{l}0.03 \\
1.8 \\
.15 \\
6.70 \\
1.40 \\
.85\end{array}$ \\
\hline & $\begin{array}{c}\text { Site number } \\
\text { and name }\end{array}$ & Late & $\begin{array}{l}\text { Sodzum, } \\
\text { dis- } \\
\text { solved } \\
\text { (mg/L) }\end{array}$ & $\begin{array}{l}\text { Sulfate } \\
\text { dis- } \\
\text { solved } \\
\text { (mg/L) }\end{array}$ & $\begin{array}{l}\text { Chlo- } \\
\text { ride, } \\
\text { dis- } \\
\text { solved } \\
(\mathrm{mg} / \mathrm{L})\end{array}$ & $\begin{array}{l}\text { Fluo- } \\
\text { ride, } \\
\text { dis- } \\
\text { solved } \\
(\mathrm{mg} / \mathrm{L})\end{array}$ & $\begin{array}{l}\text { Silica, } \\
\text { dis- } \\
\text { solved } \\
\text { (mg/t } \\
\text { as } \\
\text { Sio2) }\end{array}$ & $\begin{array}{l}\text { Total } \\
\text { dis- } \\
\text { solved } \\
\text { solids } \\
(\mathrm{mg} / \mathrm{L})\end{array}$ & $\begin{array}{l}\text { Alka- } \\
\operatorname{lin} 1 \mathrm{ty} \\
(\mathrm{mg} / \mathrm{L} \\
\text { as } \\
\text { CaCo3) }\end{array}$ & $\begin{array}{l}\text { Hard- } \\
\text { ness, } \\
\text { total } \\
(\mathrm{mg} / \mathrm{L} \\
\text { as } \\
\text { Caco3) }\end{array}$ \\
\hline \multirow[t]{2}{*}{$\begin{array}{l}\text { 1. Río } \\
\text { 2. Río } \\
\text { 3. Río } \\
\text { 4. Rio } \\
\text { 5. Rio } \\
\text { 6. Río }\end{array}$} & $\begin{array}{l}\text { Turabo at Caguas } \\
\text { Valenciano at mouth } \\
\text { Grande de Lolza at Caguas } \\
\text { Caguitas at Highway } 30 \\
\text { Balroa at mouth } \\
\text { Gurabo at Gurabo }\end{array}$ & $\begin{array}{l}07-23-87 \\
07-23-87 \\
07-22-87 \\
07-24-87 \\
07-24-87 \\
07-22-87\end{array}$ & $\begin{array}{l}25 \\
25 \\
11 \\
40 \\
27 \\
22\end{array}$ & $\begin{array}{l}28 \\
14 \\
13 \\
48 \\
15 \\
12\end{array}$ & $\begin{array}{l}26 \\
21 \\
11 \\
34 \\
26 \\
12\end{array}$ & $\begin{array}{r}0.3 \\
.2 \\
1.3 \\
.4 \\
.2 \\
.2\end{array}$ & $\begin{array}{l}44 \\
34 \\
21 \\
33 \\
32 \\
26\end{array}$ & $\begin{array}{r}300 \\
180 \\
98 \\
320 \\
270 \\
160\end{array}$ & $\begin{array}{r}186 \\
64 \\
42 \\
143 \\
170 \\
95\end{array}$ & $\begin{array}{r}190 \\
77 \\
39 \\
150 \\
170 \\
87\end{array}$ \\
\hline & $\begin{array}{l}\text { Site number } \\
\text { and name }\end{array}$ & Date & $\begin{array}{l}\text { Nitro } \\
\text { gen, } \\
\text { NO2+N } \\
\text { dis- } \\
\text { solve } \\
\text { (mg/L } \\
\text { as N) }\end{array}$ & & $\begin{array}{l}\text { Phos- } \\
\text { phorous, } \\
\text { ortho } \\
\text { dis- } \\
\text { solved } \\
\text { (mg/L } \\
\text { as P) }\end{array}$ & $\begin{array}{l}\text { Iron, } \\
\text { dis- } \\
\text { solved } \\
(\mu g / L)\end{array}$ & $\begin{array}{l}\text { Man- } \\
\text { gane- } \\
\text { se, } \\
\text { dis- } \\
\text { solved } \\
(\mu g / L)\end{array}$ & \multicolumn{2}{|c|}{$\begin{array}{l}\text { Fecal } \\
\text { coliform } \\
\text { (colonies } \\
\text { per } 100 \mathrm{ML} \\
\text { of sample) }\end{array}$} & \\
\hline $\begin{array}{l}\text { 1. Rio } \\
\text { 2. Rio } \\
\text { 3. Rio } \\
\text { 4. Rio } \\
\text { 5. Río } \\
\text { 6. Rio }\end{array}$ & $\begin{array}{l}\text { Turabo at Caguas } \\
\text { Valenclano at mouth } \\
\text { Grande de Loiza at Caguas } \\
\text { Caguitas at Highway } 30 \\
\text { Bairoa at mouth } \\
\text { Gurabo at Gurabo }\end{array}$ & $\begin{array}{l}07-23-87 \\
07-23-87 \\
07-22-87 \\
07-24-87 \\
07-24-87 \\
07-22-87\end{array}$ & $\begin{array}{l}1.5 \\
.7 \\
.39 \\
.9 \\
.1 \\
.85\end{array}$ & & $\begin{array}{r}0.02 \\
.41 \\
.07 \\
2.30 \\
.59 \\
.21\end{array}$ & $\begin{array}{r}3 \\
140 \\
220 \\
110 \\
26 \\
170\end{array}$ & $\begin{array}{r}9 \\
120 \\
33 \\
300 \\
580 \\
180\end{array}$ & & $\begin{array}{r}2200 \\
200000 \\
36000 \\
520000 \\
74000 \\
8000\end{array}$ & \\
\hline
\end{tabular}

bicarbonate type (fig. 22). The sample from well 81 was a calcium sulfate type water. The high concentrations of calcium $(380 \mathrm{mg} / \mathrm{L})$, sulfate $(1,700$ $\mathrm{mg} / \mathrm{L})$, nitrogen $(0.23 \mathrm{mg} / \mathrm{L})$, and total dissolved solids $(2,700 \mathrm{mg} / \mathrm{L})$, in this sample (well 81$)$ indicate an abnormal condition in the area near the well.

Water from most wells in the Gurabo-Juncos subarea is a calcium bicarbonate type, although some wells contained water in which no single cation-anion pair dominated the chemical composition of the water. The samples from wells 84,69 , and 29 fall into this last category (fig. 22).

Samples from streams in the area were classified as calcium bicarbonate type water. There was no notable difference between the surface water of the Caguas and Gurabo-Juncos subareas.

\section{Environmental Considerations}

Local contamination of ground and surface water of the Caguas-Juncos Valley has been reported, but little is known about the nature and extent of water-quality problems in the aquifer. There are, however, several potential sources of contamination that could affect the quality of ground water in the alluvial aquifer.

One potential threat to ground-water quality and utility is the use of underground tanks to store fuels or other substances. Given the proximity of the water-table surface to the land surface (ranging from 8 to $30 \mathrm{ft}$ in the Caguas urban area) and the design of underground storage facilities, the risk of ground-water contamination exists if the storage tanks leak. If the pressure inside the tank is higher than the pressure in the aquifer, the pressure in the tank will force the 
EXPLANATION

${ }^{32}$ O CAGUAS SUBAREA WELL AND NUMBER

89 GURABO-JUNCOS SUBAREA WELL AND NUMBER

${ }^{6} \nabla$ SURFACE-WATER SITE AND NUMBER

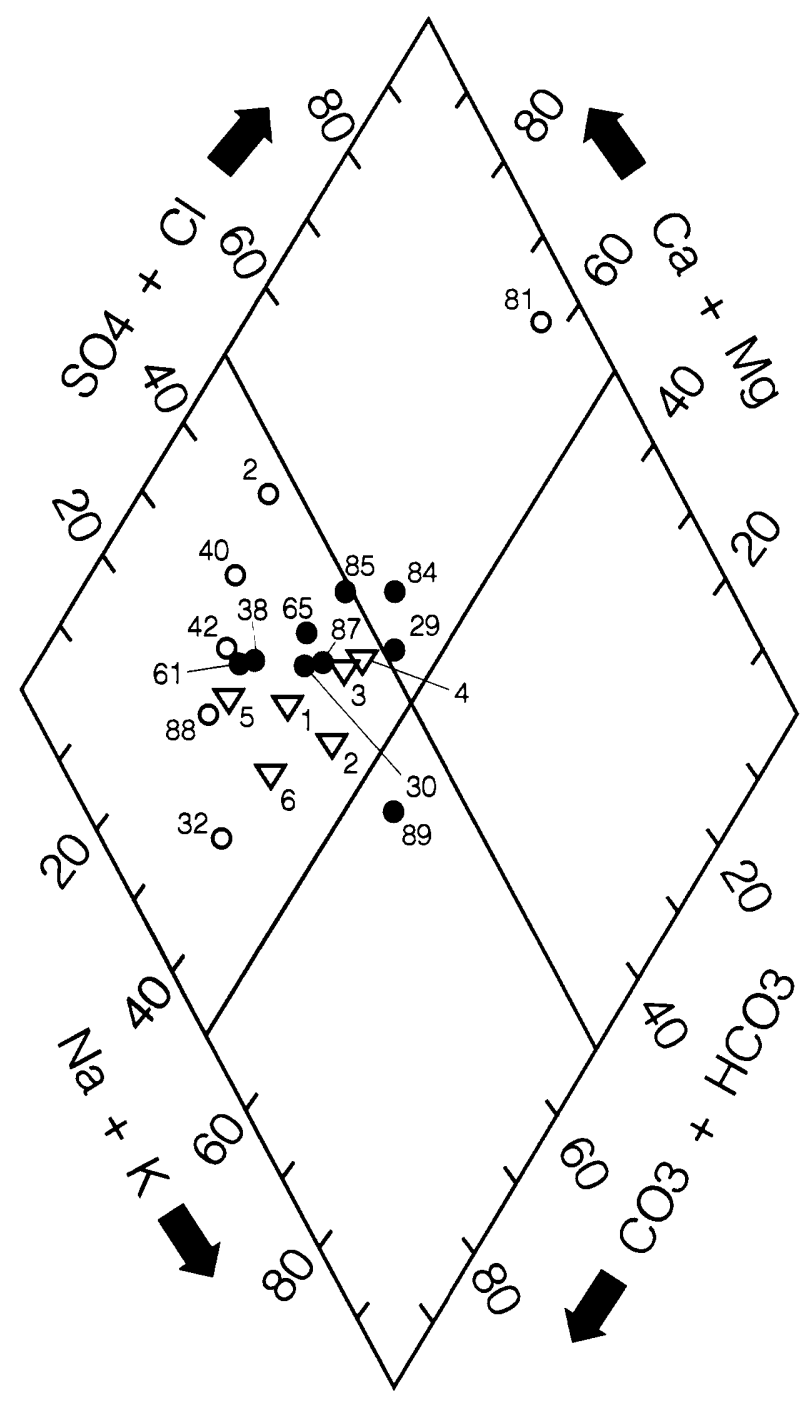

PERCENT OF TOTAL

MILLIEQUIVALENTS PER LITER

Figure 22.--Major ion composition of ground water and surface water in the study area (Refer to figure 7 for location of sample sites). 
liquids out of the tank and into the aquifer. Leaky underground storage tanks may be a worse problem in the Caguas subarea than in the less urbanized GuraboJuncos subarea.

The Caguas-Juncos Valley is a region of high agricultural activity, particularly dairy farming. Livestock wastes, which commonly are disposed of on the land surface or in ponds excavated in the unsaturated zone directly above the water-table aquifer, may leach into the aquifer. Contamination of ground water from livestock waste is more likely to be a problem in the Gurabo-Juncos subarea, where dairy farms are more numerous and are located on the alluvial flood plain, than in the Caguas subarea, where most of the dairy farms are not located in the valley.

Other agricultural activities that could result in contamination of ground water are the use of fertilizers and pesticides. The effects of this type of contamination have not been documented in this area, but the use of agricultural chemicals in the area remains a potential source of ground-water contamination.

Municipal landfills constitute an additional potential source of ground-water contamination in the Caguas-Juncos Valley, particularly those located near or within the valley boundary. In the study area, there are five landfills of which three have been closed. One of these landfills is the old Juncos landfill, which has been classified as one of the national priority cleanup sites (EPA, 1984). Although the old Juncos landfill has been closed since 1977 , it continues to be a potential source of contamination. The landfill is located outside the boundaries of the alluvial aquifer, and subsurface movement of leachate into the alluvial aquifer is not expected. Overland runoff from this landfill, however, could introduce contaminants into the aquifer down valley.

Of the five landfills in the study area, two are located almost directly above the alluvial aquifer: the Gurabo landfill (closed) near the Agricultural Experimental Station of the University of Puerto Rico and the Las Piedras landfill (active) near the Hermosura wells (fig. 7) at the eastern end of the Gurabo-Juncos subarea. Because of their location, these landfills may pose the most immediate threat to ground-water quality in the alluvial aquifer.

The effects of municipal landfills on water quality can be minimized if they are properly designed, constructed, and located. Landfills are less likely to affect the ground-water systems if they are located away from the aquifer boundaries and in relatively impermeable material, such as clay and some types of bedrock.

The type of contaminants indicative of leachates that emanate from a landfill are highly variable and include inorganic and organic compounds. The installation of a network of piezometers near and down gradient of the landfills would be necessary to quantify the effect of leachate on the quality of the ground water in the Caguas-Juncos Valley.

The waste water disposal and treatment system might also be a source of contamination. For example, in the Caguas urban area, sewer pipes lie, for the most part, directly above the principal aquifer and pipe leakage could introduce wastewater into the aquifer.

Once the wastewater is secondarily treated and discharged to a nearby stream, the wastewater again becomes a potential source of contamination. As previously stated, streams in the Caguas-Juncos Valley are hydraulically connected with the alluvial aquifer. If a river reach contains poor-quality water and that reach is losing water to the aquifer, the water quality of the surrounding aquifer can be adversely affected. This is more likely to be a problem if the most productive aquifer zones are near the streams, as they are in the Caguas-Juncos Valley.

Many communities that are not connected to the public sewer system dispose of their wastewater by means of septic tanks or sewer trenches, which discharge untreated wastewater directly into nearby creeks or streams. Septic tanks or sewer trenches constructed within the aquifer, constitute a substantial source of potential contamination to the aquifer.

Another potential source of ground-water contamination in the study area, which has not been quantitatively documented, is that related to industrial wastes. Industrial wastes have contaminated shallow aquifers in other areas of the island and may pose an increasing threat to ground-water quality as urban and industrial sites in the area expand.

\section{GROUND-WATER DEVELOPMENT AND MANAGEMENT CONSIDERATIONS}

The development and management of the ground-water resources are being considered because much of the available surface-water inflow to the Lago Loíza is exported to San Juan ( $80 \mathrm{Mgal} / \mathrm{d})$. The 
alluvial aquifer could provide an alternative source of water to supplement the available surface water, but the development of the aquifer requires a thorough understanding of the occurrence, availability, and quality of ground water in the area.

Ground-water development near streams, where the aquifer is hydraulically connected with the surface-water bodies, will increase the hydraulic gradient from the river into the aquifer and induce seepage from the river, which will improve well yield, but will decrease streamflow. A minimum river flow is necessary to sustain the required inflow into the Lago Loíza reservoir. If the amount of induced seepage from the river to the aquifer is large, maintaining this required inflow may be difficult during droughts.

An additional factor constraining the development of ground water in the area is the suitability of the water for human consumption. Production wells located near areas of contaminated or poor-quality water may not produce water suitable for public supply, unless the water undergoes extensive treatment.

The alluvial aquifer has a relatively small surface area, about $35 \mathrm{mi}^{2}$, and pumping wells may be located sufficiently close to an aquifer boundary to be affected by boundary conditions. For example, if a well is pumping near a bedrock wall, drawdowns will be greater and pumping costs higher than if the well were located near a stream.
The selection of a ground-water withdrawal strategy in the study area is also subject to additional limiting factors:

1) If ground-water supply wells are located in high transmissive zones, away from contaminated areas, any adverse effects on the aquifer due to migration of contaminated water to pumping centers will be minimized.

2) Withdrawals may have to be restricted to ensure that seepage from the rivers induced by pumping does not reduce the minimum baseflow of the stream below the baseflow required to sustain the Lago Loíza reservoir.

3) Seasonal hydrologic variations may affect the ground-water withdrawal schedule. During most of the year, rivers have enough flow to supply the Lago Loíza, as well as production wells, but withdrawals may have to be restricted during low-flow periods and droughts.

4) Development of the ground-water resources needs to be monitored to prevent exploitation of the aquifer. Withdrawal from the aquifer in excess of the long-term, average natural recharge will result in depletion of the resource.

The factors presented are generalized, and other local factors may also be important to the development of a specific water supply. Quantitative analyses best performed with numerical flow models are beyond the scope of this study. However, this study provides much of the data required to construct and calibrate such models. 


\section{SUMMARY AND CONCLUSIONS}

The Caguas-Juncos Valley is the laigest interior valley in Puerto Rico, and covers about $35 \mathrm{mi}^{2}$. The municipalities of Caguas, Gurabo, and Juncos are located within the valley and had a combined population of about 167,000 in 1980 . Urban population in the valley is expected to be 192,000 by 1990. The Caguas subarea is semi-urban (40 percent) and the Gurabo-Juncos subarea is mostly pasture land (90 percent).

All of the streams in the valley drain to the Lago Loíza and much of the inflow to the Lago Loíza is exported to San Juan for public-water supply. Unless an alternative water source is developed for the San Juan area, increased water demand in the Caguas-Juncos area may have to be satisfied by developing the ground-water resources within the valley.

Precipitation in the upper Río Grande de Loíza basin is sufficient to support perennial streams. Rainfall ranges from more than $100 \mathrm{in} / \mathrm{yr}$ in the mountains, to about $70 \mathrm{in} / \mathrm{yr}$ in the Caguas-Juncos Valley. The estimated potential evapotranspiration in the drainage basin ranges from 34 to $48 \mathrm{in} / \mathrm{yr}$. Within the valley itself, however, the evapotranspiration rate using the steady-state water balance equations was estimated to be about $7 \mathrm{in} / \mathrm{yr}$ in the Caguas subarea and about $31 \mathrm{in} / \mathrm{yr}$ in the Gurabo-Juncos subarea.

The average discharges for the period 1985 to 1988 at the long-term gaging stations in the Río Grande de Loíza, the Río Gurabo, the Río Valenciano were $268 \mathrm{ft}^{3} / \mathrm{s}, 178 \mathrm{ft}^{3} / \mathrm{s}$, and $69 \mathrm{ft}^{3} / \mathrm{s}$, respectively. The highest measured flood in the valley occurred on September 6, 1960; peak discharges were about 71,500 $\mathrm{ft}^{3} / \mathrm{s}$ at the Río Grande de Loíza station and $60,000 \mathrm{ft}^{3} / \mathrm{s}$ at the Río Gurabo station. The 7-day, 10-year low flow was $14 \mathrm{ft}^{3} / \mathrm{s}$ at the surface-water stations on the Río Grande de Loíza, $6.8 \mathrm{ft}^{3} / \mathrm{s}$ at the station on the Río Gurabo, and $3.3 \mathrm{ft}^{3} / \mathrm{s}$ at the Río Valenciano station.

The main use of ground water in the CaguasJuncos Valley is for public supply, but pumpage is relatively small, about $3.0 \mathrm{Mgal} / \mathrm{d}$, which represents about 10 percent of the total water use in the area. Other users of ground water include dairy farms, industry, and households.

Well yields in the Caguas-Juncos Valley range from 10 to $310 \mathrm{gal} / \mathrm{min}$. Most of the high-yield wells are public-supply wells operated by the PRASA. Wells used for dairy farm operations generally have low yields (about $30 \mathrm{gal} / \mathrm{min}$ ).

The potential for ground-water development in the Caguas-Juncos Valley is higher within the alluvial deposits. The thickness of the alluvial deposits is variable, but averages about $45 \mathrm{ft}$. In general, the alluvial deposits tend to be thicker and more permeable in the Gurabo-Juncos subarea. In the Caguas subarea, the alluvial deposits are thinner and have a higher clay content.

Estimates of transmissivity in the area range between 66 and $4,770 \mathrm{ft}^{2} / \mathrm{d}$, and average $1,350 \mathrm{ft}^{2} / \mathrm{d}$. Transmissivity values generally are lower in the Caguas area than in the Gurabo-Juncos area. Transmissivity values of $2,000-3,000 \mathrm{ft}^{2} / \mathrm{d}$ are common in the Gurabo-Juncos subarea, but are less than 1,000 $\mathrm{ft}^{2} / \mathrm{s}$ in the Caguas subarea. Specific-yield values are estimated to be between 10 and 15 percent.

Ground water in the alluvial aquifer generally is unconfined. The aquifer is in good hydraulic connection with the streams traversing the valley. Additional aquifer recharge might be induced from the streams if wells nearby are pumped.

The direction of regional ground-water flow in the Caguas subarea is from southwest to northeast. In the Gurabo-Juncos subarea, regional ground-water flow is toward the Río Gurabo and from east to west. The Caguas and the Gurabo-Juncos subareas merge just upstream of the Lago Loíza. The net ground-water seepage to streams in the Caguas-Juncos Valley in March 1988 was about $23 \mathrm{ft}^{3} / \mathrm{s}$. In the Caguas subarea, the average hydraulic gradient in the alluvial aquifer was $49 \mathrm{ft} / \mathrm{mi}$, while in the Gurabo-Juncos subarea it was about $28 \mathrm{ft} / \mathrm{mi}$.

Continuous and periodic ground-water level records at selected wells indicate that seasonal ground-water level fluctuations generally are between 1.5 and $11.0 \mathrm{ft}$, and average about $4 \mathrm{ft}$. Ground-water levels generally are low in April and September and high in December.

The amount of water in storage in the alluvial aquifer was estimated to be about 46,700 acre- $\mathrm{ft}$ in the Caguas subarea and about 75,200 acre- $\mathrm{ft}$ in the Gurabo-Juncos subarea. The steady-state ground-water budget analysis for the Caguas and the Gurabo-Juncos subareas revealed that the main sources of water to the aquifer are rainfall infiltration $(6,100$ acre- $\mathrm{ft} / \mathrm{yr}$ in Caguas and 5,600 acre-ft/yr in Gurabo-Juncos) and 
bedrock seepage $(5,400$ acre-ft/yr in Caguas and 5,000 acre-ft/yr in Gurabo-Juncos). Most of the net outflow from the aquifer occurs as stream seepage, 11,300 acre- $\mathrm{ft}$ in Caguas and 7,500 acre-ft/yr in Gurabo-Juncos.

Ground water and surface water in the Caguas-Juncos Valley is a calcium-bicarbonate type. Locally, ground-water in the valley has high concentrations of iron and manganese. Concentrations of iron and manganese greater than $0.3 \mathrm{mg} / \mathrm{L}$ and 0.05 $\mathrm{mg} / \mathrm{L}$ were observed at nine of the 18 wells sampled in 1987.
The values for other physical and chemical characteristics were elevated in samples from some wells. For example, specific-conductance values greater than $1,000 \mu \mathrm{S} / \mathrm{cm}$ were detected at three wells and the sulfate concentration in one well was 1,700 $\mathrm{mg} / \mathrm{L}$. Some PRASA wells were monitored for the presence of volatile organics. The water-quality samples collected in December 1988, indicated that only one well (well 43) had detectable concentrations of chloroform $(67 \mu \mathrm{g} / \mathrm{L})$. All surface-water samples collected at six selected streams sites contained fecal coliform bacteria and had concentrations as high as $520,000 \mathrm{col} . / 100 \mathrm{ml}$. 


\section{REFERENCES}

Bennett, G.D., 1976, Electrical analog simulation of the aquifers along the south coast of Puerto Rico: U.S. Geological Survey Open-File Report 76-4, 101 p.

Bouwer, Herman, and Rice, R.C., I976, A slug test for determining hydraulic conductivity of unconfined aquifers with completely or partially penetrating wells: Water Resources Research, v. 12, no. 3, p. 423-428.

Broedel, C.H., 1961, Preliminary geologic map showing iron and copper prospects in the Juncos quadrangle, Puerto Rico: U.S. Geological Survey Miscellaneous Geologic Investigations Report, Map I-326, scale 1:20,000

Colón-Dieppa, Eloy, and Quiñones-Aponte, Vicente, 1985, Estimates of 7-day, 10-year low flow at ungaged streams in Puerto Rico: U.S. Geological Survey Water-Resources Investigations Report 84-4089, 1 pl.

Crooks, J.W., Grossman, I.G., and Bogart, D.B., 1968, Water resources of the Guayanilla-Yauco area, Puerto Rico: U.S. Geological Survey Water-Resources Bulletin 5, 55 p.

Curtis, R.E., Jr., Aquino, Zaida, Díaz, P.L., and Vachier, Ricardo, 1989, Water resources data, Puerto Rico and the U.S. Virgin 1slands, water year 1987: U.S. Geological Survey Water-Data Report PR-87-1, 356 p.

Díaz, J.R., and Jordan, D.G., 1987, Water resources of the Río Grande de Añasco lower valley, Puerto Rico: U.S. Geological Survey Water-Resources Investigations Report 85-4237, 48 p.

Fields, F.K., 1972, Floods at Caguas, Gurabo, Juncos, and San Lorenzo, Puerto Rico: U.S. Geological Survey Hydrologic Investigations Atlas HA-438.

Freeze, R.A., and Cherry, J.A., 1979, Groundwater: Prentice-Hall Inc., Englewood Cliffs, N.J., 604 p.

Giusti, E.V., and López, G.D., 1967, Climate and streamflow of Puerto Rico: Caribbean Journal of Science, September-December 1967, v. 7, no. 3-4, p. $87-93$.
Gómez-Gómez, Fernando, and Guzmán-Ríos, Senén, 1982, Reconnaissance of ground-water quality throughout Puerto Rico, September - October 1981: U.S. Geological Survey Open-File Report 82-332, I pl.

Guzmán-Ríos, Senén, García, René, and Avilés, Ada, I986, Reconnaissance of volatile synthetic organic chemicals at public supply wells throughout Puerto Rico, November I984 - May 1985: U.S. Geological Survey Open-File Report 86-63, 1 pl.

Guzmán-Ríos, Senén, and Quiñones-Márquez, Ferdinand, 1984, Reconnaissance of ground-water quality at selected sites throughout Puerto Rico, September 1982 - July 1983: U.S. Geological Survey Open-File Data Report 84-058, 1 pl.

1985, Reconnaissance of trace organic compounds in ground water throughout Puerto Rico, October 1983: U.S. Geological Survey Open-File Data Report 84-810, $1 \mathrm{pl}$.

Jordan, D.G., and Fisher, D.W., 1977, Relation of bulk precipitation and evapotranspiration to water quality and water resources, St. Thomas, U.S. Virgin Islands: U.S. Geological Survey Water-Supply Paper 1663-I, $30 \mathrm{p}$.

Lohman, S.W., and others, 1972, Definitions of selected ground-water terms - Revisions and Conceptual Refinements: U.S. Geological Survey Water-Supply Paper 1988, 21 p.

McGonigle, J.W., 1978, Geologic Map of the Humacao quadrangle, Puerto Rico: U.S. Geological Survey Miscellaneous 1nvestigations Series, Map 1-1070, scale 1:20,000.

Pease, Jr., M.H., 1968, Geologic map of the Aguas Buenas quadrangle, Puerto Rico: U.S. Geological Survey Miscellaneous Geologic Investigations Series, Map 1-479, scale 1:20,000.

Piper, A.M., 1953, A graphic procedure in the geochemical interpretation of water analyses: U.S. Geological Survey, Ground Water Notes, Geochemistry, no. 12, 14 p. 


\section{REFERENCES--Continued}

Puerto Rico Planning Board, 1984, Proyecciones de población por municipio, 1985-2005, Area de Planificación Económica y Social, 3 p.

Quiñones-Aponte, Vicente, 1986, Simulation of ground-water flow in the Río Yauco alluvial valley, Yauco, Puerto Rico: U.S. Geological Survey Water-Resources Investigations Report 85-4179, $32 \mathrm{p}$.

Quiñones-Márquez, Ferdinand, 1980, Limnology of Lago Loíza, Puerto Rico: U.S. Geological Survey Water-Resources Investigations Report 79-97, $114 \mathrm{p}$.

Quiñones, Ferdinand, Colón-Dieppa, Eloy, and Juarbe, Max, 1984, Flow duration at streamflow gaging stations in Puerto Rico: U.S. Geological Survey Open-File Data Report 84-127, 93 p.

Quiñones, Ferdinand, Green, Bruce, and Santiago, Luis, 1989, Sedimentation survey of Lago Loíza, Puerto Rico, July 1985: U.S. Geological Survey Water-Resources Investigations Report 87-4019, $17 \mathrm{p}$.
Rogers, C.L., 1979, Geologic map of the Caguas quadrangle, Puerto Rico: U.S. Geological Survey Miscellaneous Investigations Series, Map I-1152, scale 1:20:000.

Seiders, V.M., 1971, Geologic map of the Gurabo quadrangle, Puerto Rico: U.S. Geological Survey Miscellaneous Geologic Investigations Series, Map I-657, scale 1:20,000.

Theis, C.V., Brown, R.H., and Meyer, R.R., 1963, Estimating the transmissivity of aquifers from the specific capacity of wells, in R. Bentall, Methods of Determining Permeability, Transmissibility and Drawdown: U.S. Geological Survey Water-Supply Paper 1536-I, p. 331-341.

Todd, D.K, 1980, Groundwater hydrology: John Wiley \& Sons, Second Edition, New York, 535 p.

U.S. Environmental Protection Agency, 1973, Water Quality Criteria 1972, Committee on Water Quality Criteria, EPA-R3-73-003, 594 p.

1984, Region II, Administrative order and consent, Index number II-CERCLA-40303, October 9, 1984. 